Aus der Abteilung Präventive Zahnmedizin, Parodontologie und Kariologie

(Komm. Direktor: Prof. Dr. med. dent. M. Hülsmann)

im Zentrum Zahn-, Mund- und Kieferheilkunde

der Medizinischen Fakultät der Universität Göttingen

\title{
Klinische Studie zur Überprüfung des Einflusses der Gebrauchsdauer verschiedener elektrischer Zahnbürsten auf die mechanische Plaquekontrolle und den gingivalen Zustand
}

\author{
INAUGURAL - DISSERTATION \\ zur Erlangung des Doktorgrades \\ für Zahnheilkunde \\ der Medizinischen Fakultät \\ der Georg-August-Universität zu Göttingen \\ vorgelegt von \\ Sabine Wurbs \\ aus Kassel
}

Göttingen 2011 
Dekan: Prof. Dr. med. C. Frömmel

1. Berichterstatter: Prof. Dr. med. dent. R. F. Mausberg

2. Berichterstatter/in:

3. Berichterstatter/in:

Tag der mündlichen Prüfung: 
Abkürzungsverzeichnis

ADA American Dental Association

API Approximalraum-Plaque-Index

DMF-T Index zur Erfassung der kariösen (D decayed), fehlenden (M missing)

und gefüllten (F filled) Zähne (T teeth) (KLEIN et al. 1938)

GI Gingivaindex (LÖE und SILNESS 1963)

HIV Humanes Immundefizienz-Virus

MW Mittelwert

PBI Papillen-Blutungs-Index (SAXER und MÜHLEMANN 1975)

PEP-PTS Phosphoenolpyruvat-Phosphotransferasesystem

$\mathrm{pH}$ pondus Hydrogenii oder potentia Hydrogenii (lat. Pondus = Gewicht, lat. Potentia $=$ Kraft, lat. Hydrogenium $=$ Wasserstoff $)$

PI Plaqueindex

ppm parts per million

p-Wert $\quad$ von p-value (engl. probability $=$ Wahrscheinlichkeit)

PZR professionelle Zahnreinigung

QHI Quigley-Hein-Index (QUIGLEY und HEIN 1962) modifiziert nach Turesky (TURESKY et al. 1972)

S. Streptococcus

SBI Sulkus-Blutungs-Index (MÜHLEMANN und SON 1971)

SD Standardabweichung

TBC Tuberkulose

UV ultraviolett 
Inhaltsverzeichnis

1 Einleitung und Zielparameter ............................................................................................... 1

1.1 Einleitung ........................................................................................................................... 1

2 Literaturübersicht ..........................................................................................................

2.1 Plaque bzw. Biofilm............................................................................................................ 3

2.2 Ätiologie der Karies ............................................................................................... 6

2.3 Ätiologie der entzündlichen Parodontopathien ...................................................................9

2.4 Bedeutung der Prophylaxe ..................................................................................................... 11

2.5 Prophylaxeinstrument Zahnbürste ................................................................................... 12

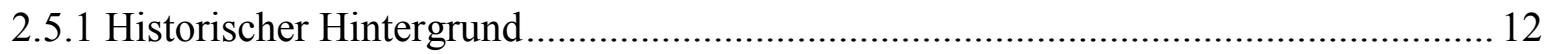

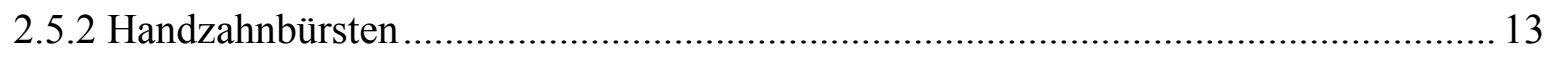

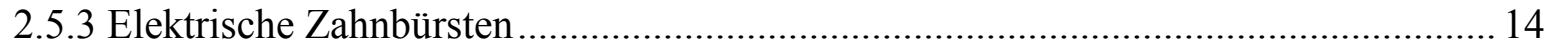

2.5.4 Elektrisch unterstützte Handzahnbürsten ................................................................ 15

2.6 Effektivität elektrischer Zahnbürsten im Vergleich zu Handzahnbürsten ................. 15

2.7 Effektivität rotierend-oszillierender Zahnbürsten im Vergleich mit

Schallzahnbürsten ................................................................................................................ 16

2.8 Wann sollte eine Zahnbürste ausgewechselt werden? ..................................................... 17

2.8.1 Ineffizienz abgenutzter Borsten bei der Plaquereduktion ......................................... 19

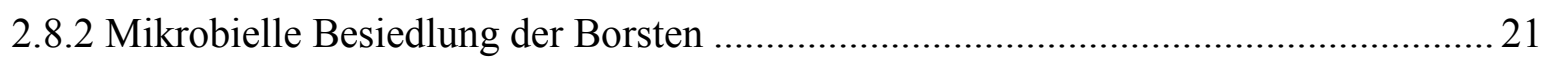

2.8.3 Verletzungsgefahr durch abgenutzte Borsten ...................................................... 22

2.8.4 Abnutzung, Reinigungswirkung und Bürstenkopfwechsel bei elektrischen

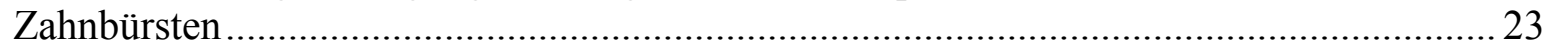

3 Material und Methoden ............................................................................................... 24

3.1 Administrative Vorbereitungen .................................................................................... 24

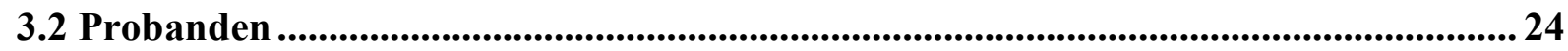

3.3 Testzahnbürsten und Testzahnpasta ............................................................................ 24

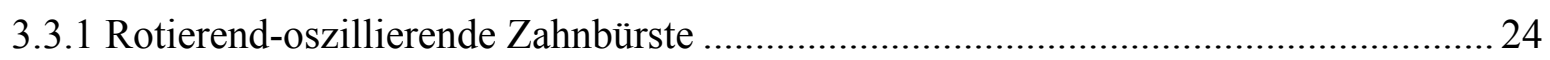

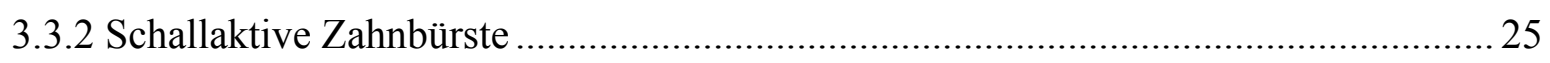

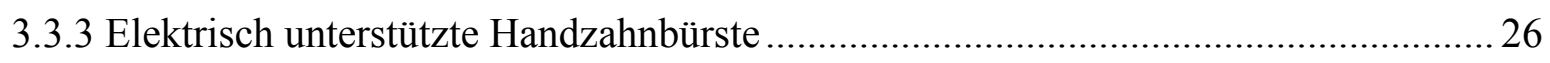

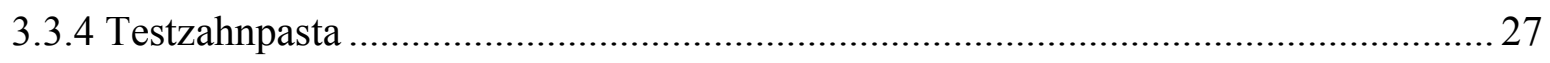

3.4 Mundhygiene-Indizes und Plaquerelevator .................................................................. 28

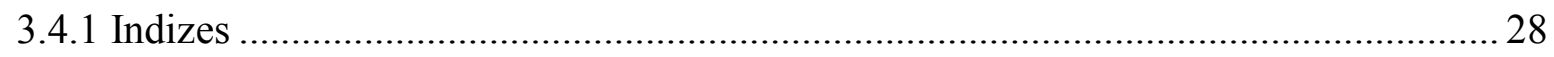

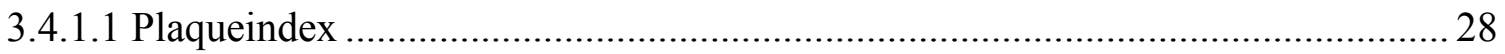

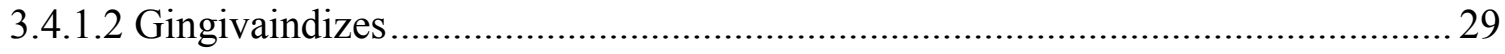

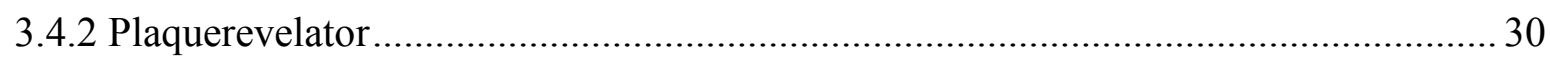

3.5 Einteilung der Gruppen ....................................................................................................30

3.6 Studiendesign ...................................................................................................................... 31

3.7 Studienablauf .........................................................................................................................31

3.7.1 Anamnese und Einverständniserklärung ………………........................................ 31 


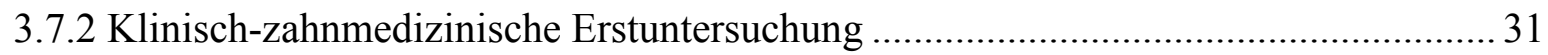

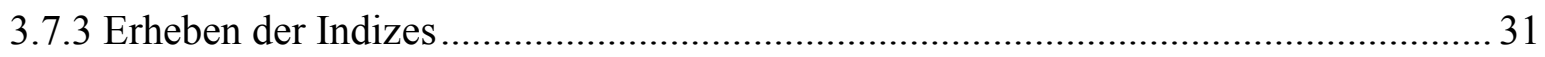

3.8 Randomisierung und Instruktion der Probanden/Zahnreinigung............................. 31

3.9 Untersuchungs- und Kontrollintervall .................................................................................. 32

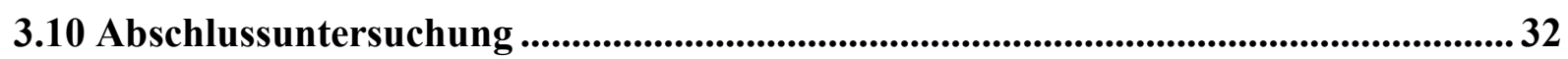

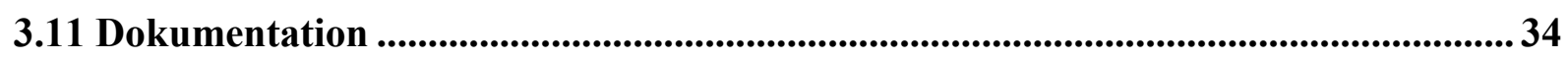

3.12 Auswertung der Ergebnisse.................................................................................................... 34

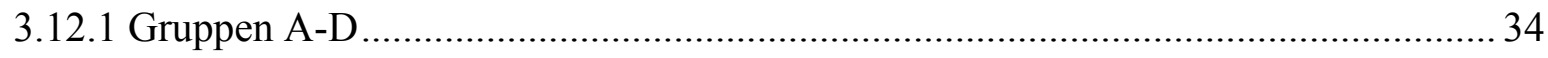

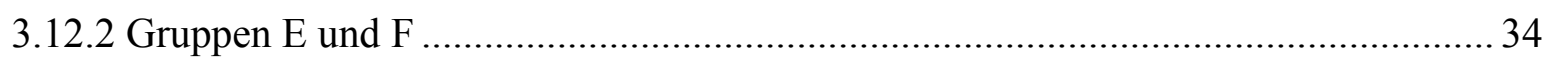

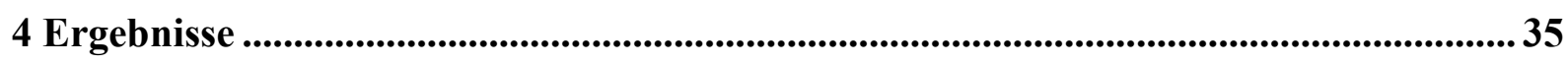

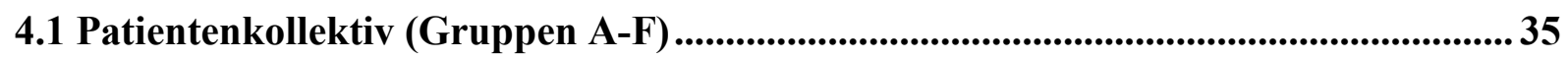

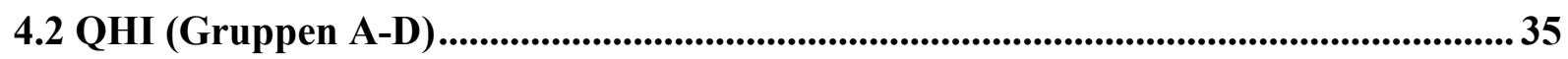

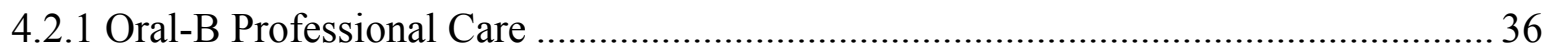

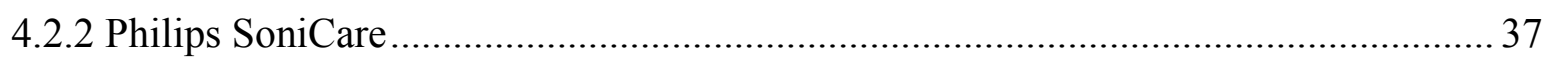

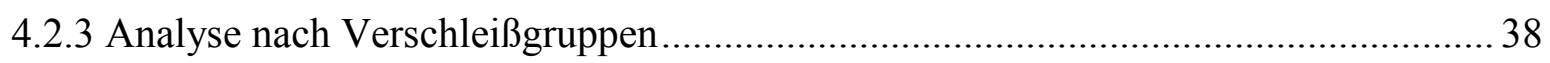

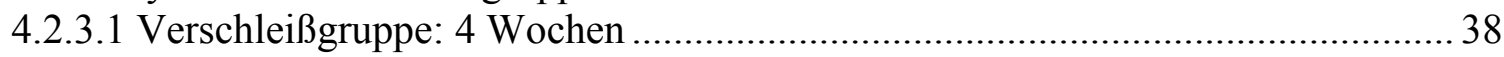

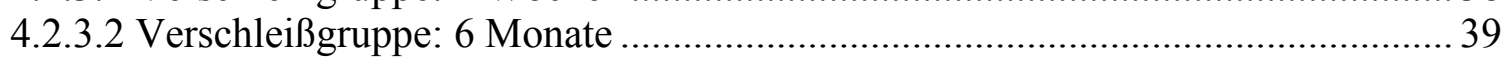

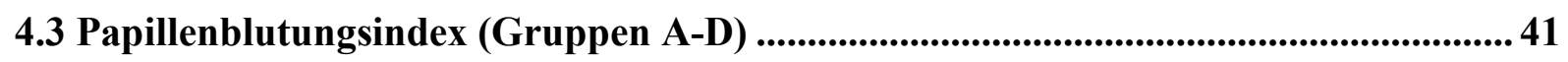

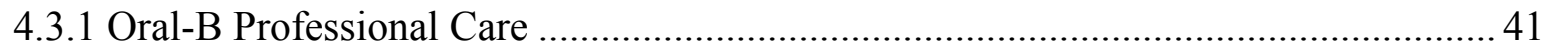

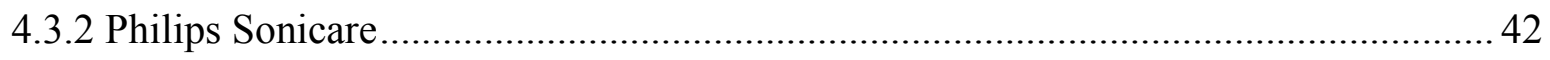

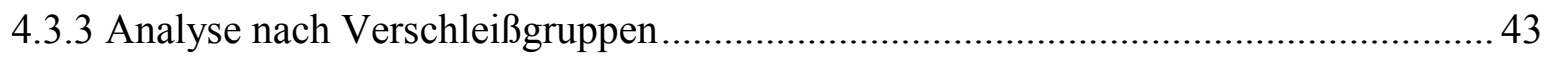

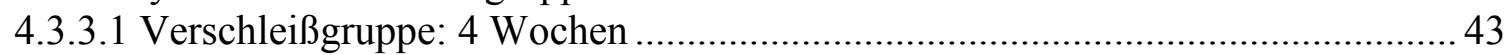

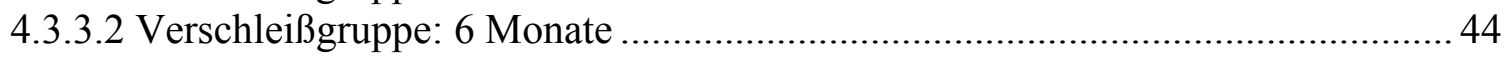

4.4 Gingivaindex nach Löe und Silness (Gruppen A-D)...............................................46

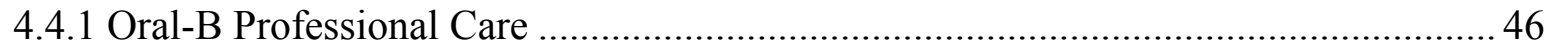

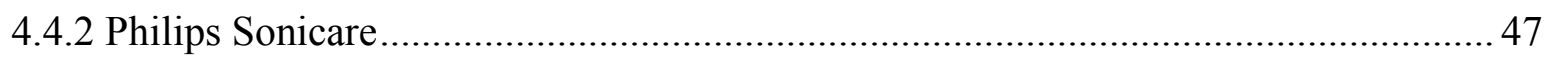

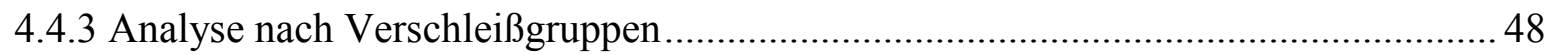

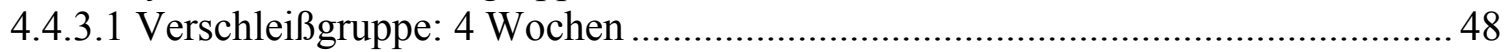

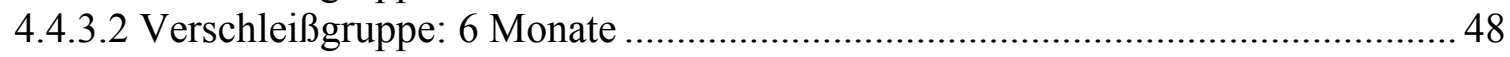

4.5 Zusammenfassung der Ergebnisse (Gruppen A-D) .....................................................50

4.6 Ergebnisse der elektrisch unterstützten Handzahnbürsten (Gruppen E und F).......51

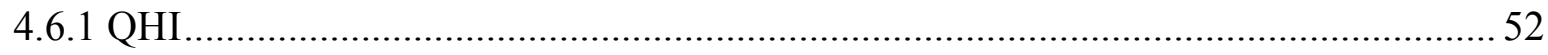

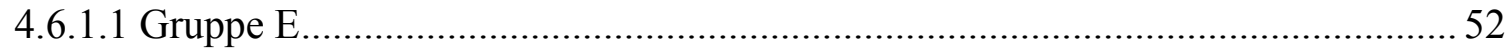

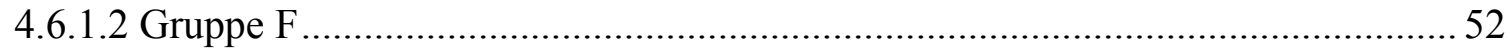

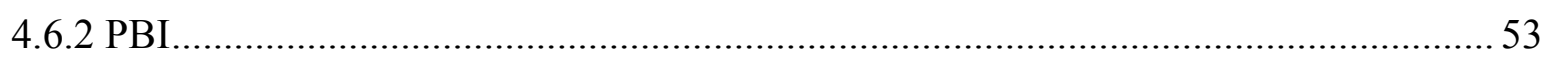

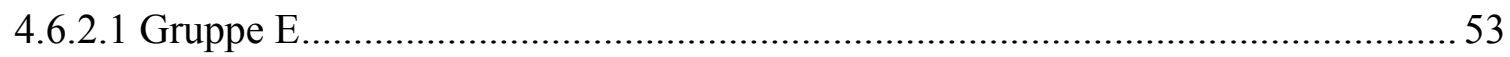

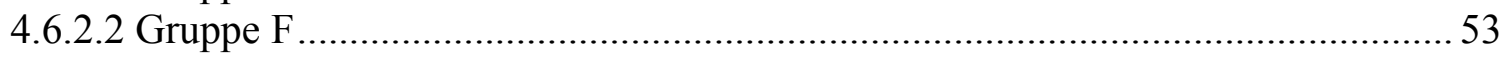

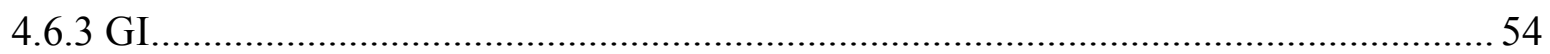

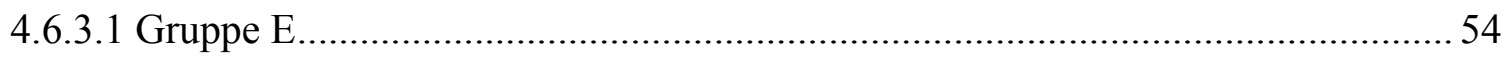

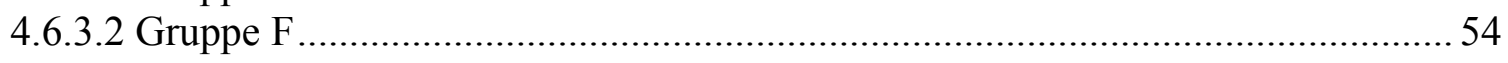

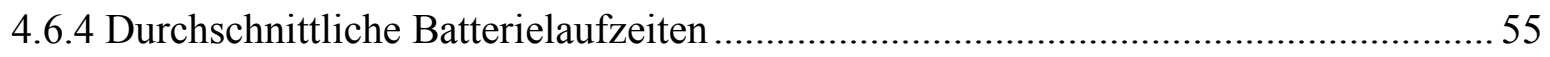

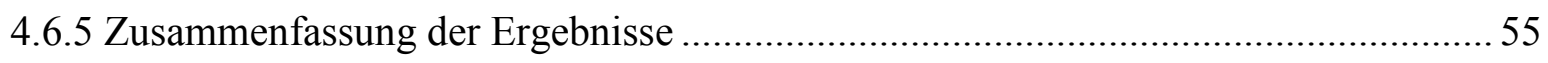


5 Diskussion ..................................................................................................................................... 56

5.1 Studiendesign ................................................................................................................................... 56

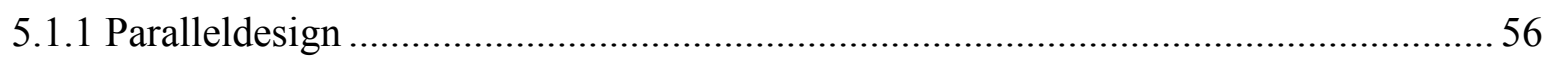

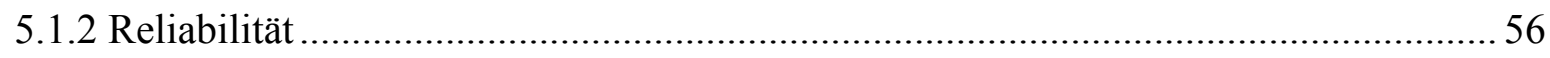

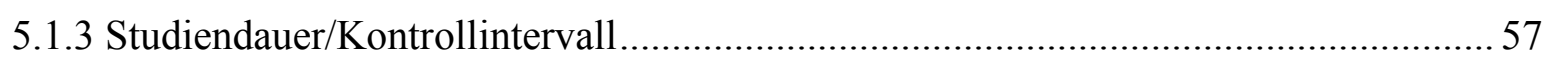

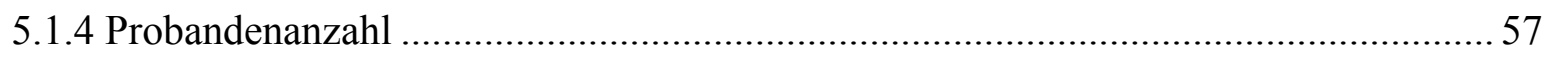

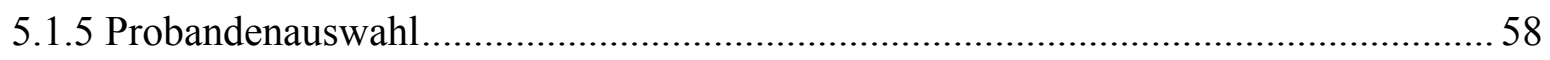

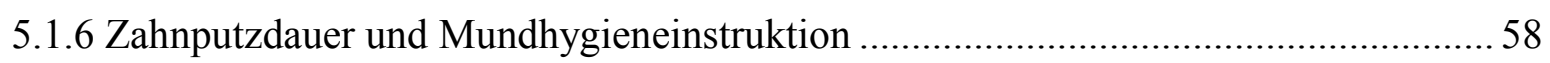

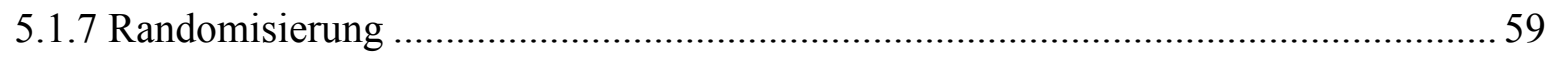

5.2 Material .......................................................................................................................................5 59

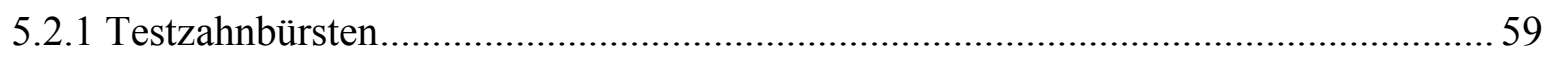

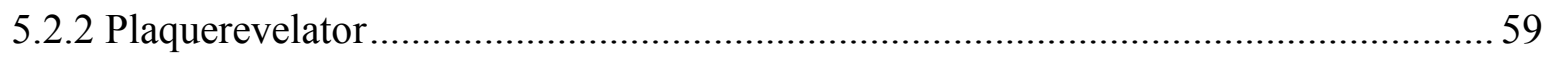

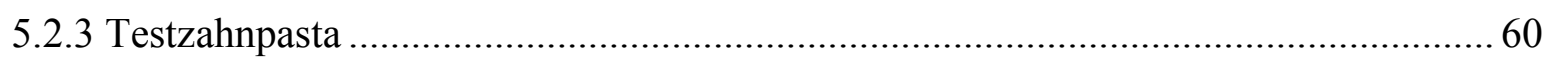

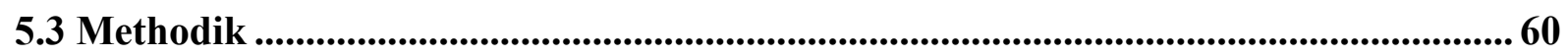

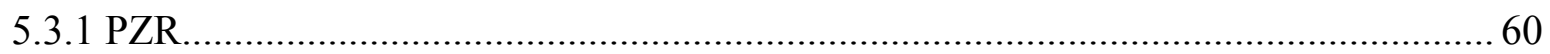

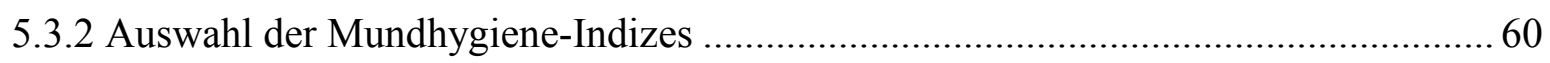

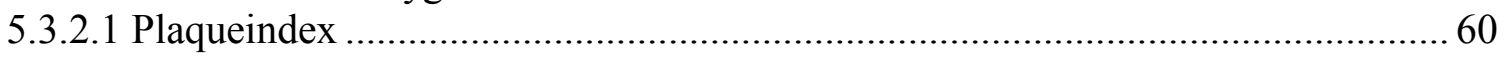

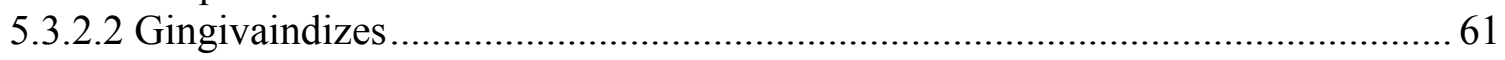

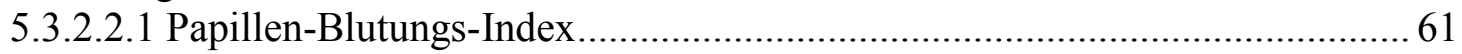

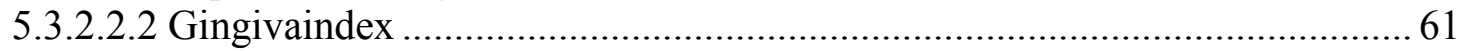

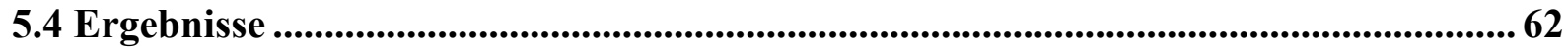

5.4.1 Rotierend-oszillierende und schallaktive Zahnbürste (Gruppen A-D) ........................ 62

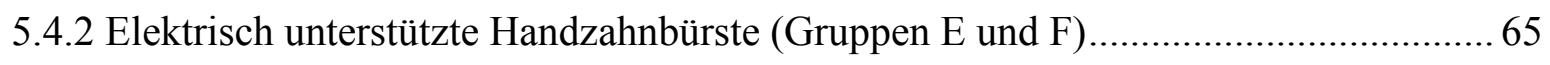

6 Zusammenfassung............................................................................................................... 67

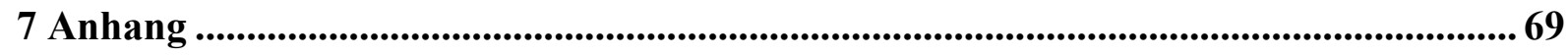

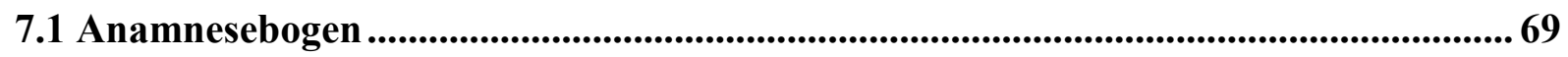

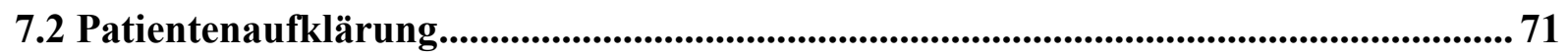

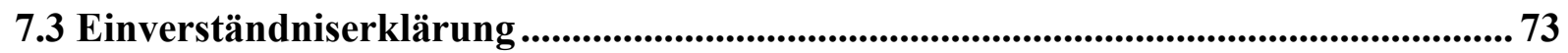

7.4 Dokumentationsbögen zur Erhebung der Indizes....................................................... 74

7.5 Excel-Tabelle zur statistischen Auswertung der Indizes ........................................... 76

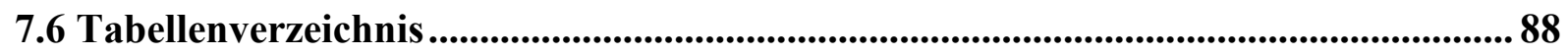

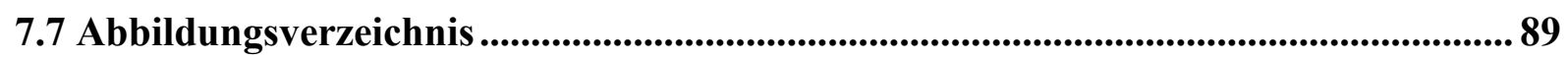

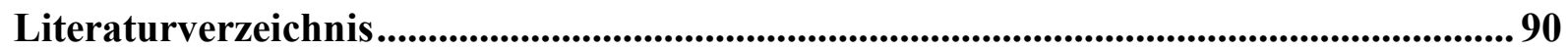




\section{Einleitung und Zielparameter}

\subsection{Einleitung}

In der Ätiologie der Karies und der entzündlich bedingten Parodontopathien stellt die bakterielle Plaque einen wichtigen Faktor dar. Es besteht ein Zusammenhang zwischen mikrobiellen Zahnbelägen und entzündlichen Veränderungen des Parodontiums (LÖE et al. 1965, MÜLLER 2001, RATEITSCHAK et al. 2004). Dentale Plaque ist ein strukturierter, zäher Biofilm, der sich aus Bakterien, bakteriellen Stoffwechselprodukten, Speichel- und Nahrungsbestandteilen zusammensetzt. Dieser Biofilm lässt sich nicht durch die Selbstreinigungskräfte der Mundhöhle entfernen; eine effektive Entfernung der Plaque ist vielmehr ausschließlich mechanisch, das heißt durch die Verwendung von Mundhygienehilfsmitteln wie Zahnbürste, Zahnpasta und Zahnseide, zu erreichen (HEIDEMANN 1997, HELLWIG et al. 1999, SFORZA et al. 2000). Um den Mundhygienezustand eines Patienten objektiv beurteilen zu können, bzw. um die Reinigungseffizienz einer Zahnbürste zu bewerten, ist es notwendig, sogenannte Plaque- und Gingivaindizes zu erheben, welche reproduzierbare, qualitative und quantitative Aussagen erlauben.

Seit etwa 15 Jahren belegen Studien, dass mit elektrischen Zahnbürsten eine bessere Plaquereduktion erreicht werden kann als durch die Verwendung von Handzahnbürsten. Rotierend-oszillierende Bürstensysteme und Schallzahnbürsten reinigten am Zahnfleischrand und im Approximalraum besser als Handzahnbürsten (LANG et al. 1998, LAZARESCU et al. 2003, HIMMER und EICKHOLZ 2008). In der Literatur besteht jedoch derzeit noch Uneinigkeit darüber, ob ein weniger lang gebrauchter, nicht abgenutzter Bürstenkopf generell effizienter reinigt als ein älterer, bereits abgenutzter Bürstenkopf. Im Allgemeinen wird ein Zahnbürstenwechsel nach etwa drei Monaten empfohlen. Ein konkretes Zeitintervall zum Austausch abgenutzter Zahnbürsten kann aufgrund der Beeinflussung der Abnutzung durch individuelle Faktoren wie Putztechnik und angewendete Kraft nicht allgemeingültig definiert werden.

In dieser Studie sollte untersucht werden, ob die Reinigungswirkung verschiedener elektrischer Zahnbürsten mit deren Gebrauchsdauer korreliert.

\subsection{Zielparameter}

Ziel der vorliegenden Arbeit war die Untersuchung des Einflusses der Gebrauchsdauer verschiedener elektrischer Zahnbürsten auf die mechanische Plaquekontrolle und den gingivalen 
Zustand. Dabei sollte die Hypothese, dass die Gebrauchsdauer einer Zahnbürste die Plaqueentfernung beeinflusst, überprüft werden. 


\section{Literaturübersicht}

\subsection{Plaque bzw. Biofilm}

Dentale Plaque ist ein zäher, verfilzter Zahnbelag (Biofilm), der sich aus Bakterienzellen, bakteriellen Stoffwechselprodukten, Speichelbestandteilen und Nahrungsresten zusammensetzt. Sie ist das Produkt bakterieller Kolonisation und Proliferation.

Plaquebakterien zeigen eine strukturelle Organisation; sie sind in eine organische Matrix, bestehend aus Speichelglykoproteinen und extrazellulären Polysacchariden, eingebettet. Zu 8090\% besteht der Biofilm aus Wasser; 70\% des Trockengewichtes machen Bakterienzellen aus. Aufgrund ihrer speziellen Architektur ist ausgereifte Plaque durch die Selbstreinigungskräfte der Mundhöhle wie Speichelfluss, Zungen- und Wangenbewegungen nicht von den Zähnen zu entfernen (NOLDEN 1994). Man unterscheidet zwischen supragingivaler und subgingivaler Plaque. Während supragingivale Plaque den entscheidenden ätiologischen Faktor für die Entwicklung von Karies und Gingivitis darstellt, ist die subgingivale Plaque Ursache für die Entstehung von Parodontitiden (LEHMANN und HELLWIG 1998, HELLWIG et al. 1999).

\section{Entwicklung und Reifung der Plaque}

Die Entwicklung des Biofilms vollzieht sich in vier Phasen (RENGGLI 1990, THEILADE 1984):

\section{Pelliclebildung}

Unmittelbar nach der Reinigung der Zähne, innerhalb von Minuten bis zwei Stunden, bildet sich auf deren Oberfläche das sogenannte exogene Zahnoberhäutchen (,acquired pellicle“).

Dieser initiale orale Biofilm ist maximal $1 \mu \mathrm{m}$ dick, azellulär und histologisch nicht strukturiert. Er besteht vorwiegend aus Proteinen des Speichels und der Sulkusflüssigkeit, deren Eigenladungen eine elektrostatische Verbindung an die Apatitkristallite des Zahnschmelzes ermöglichen (HELLWIG et al. 1999). Das Pellicle stellt ein Ionendepot für die Remineralisation der Zahnhartsubstanzen dar, schützt den Zahn vor Austrocknung und dient als Erosionsschutz (PLAGMANN 1998). Reibungskräfte, wie sie beispielsweise durch den Kontakt antagonistischer Zähne oder durch den Kontakt der Zahnoberflächen mit der Mukosa auftreten, werden durch das exogene Schmelzoberhäutchen reduziert (AGUIRRE et al. 1987). Man unterscheidet eine frühe und eine reife Form des Pellicles (PLAGMANN 1998). Grundlage für die Entstehung des frühen Pellicles („early pellicle“) ist die selektive Adsorption von Molekü- 
len aus dem Speichel (Proteine, Glykoproteine und Muzin) und Plasmabestandteilen, aber auch von bakteriellen Proteinen. Zum Teil treten hier auch Bestandteile aus anderen Quellen auf, wie beispielsweise Albumin aus dem Sulkusfluid (SCANNAPIECO und LEVINE 1990). Spontane Adsorptionsmechanismen laufen innerhalb weniger Sekunden ab und kennzeichnen den ersten Schritt der Pelliclebildung. Hierbei interagieren Biomoleküle mit der Zahnhartsubstanz, das Resultat ist ein wenige Nanometer dünner Film (HANNIG und HANNIG 2007). Bei den Bindungsmechanismen zwischen Speichelmolekülen und Zahnhartsubstanz handelt es sich um Kombinationen aus physikalischen Kräften durch Ionenbindungen, hydrophoben Wasserstoffbindungen sowie van-der-Waals-Kräften. Die molekulare Struktur der Speichelproteine erfährt hierdurch eine Veränderung, was wiederum eine bakterielle Anhaftung begünstigt (PLAGMANN 1998). Durch Ein- bzw. Auflagerung bakterieller Stoffwechselprodukte sowie weiterer Komponenten aus dem Sulkusfluid entsteht das reife Pellicle („later pellicle“).

\section{Phase 1 (0-2. Tag)}

Innerhalb der ersten zwei bis vier Stunden nach Zahnreinigung erfolgt zunächst die Anlagerung erster grampositiver Kokken aus dem Speichel bzw. aus Schmelzunregelmäßigkeiten. Zu etwa 90\% bilden vorwiegend Streptococcus (S.) mitis, S. sanguis, S. salivarius, später auch S. mutans, die ersten Streptokokken-Kolonien. Vereinzelt finden sich in dieser Phase auch stäbchenförmige Bakterien, meist Actinomyces-Spezies (THEILADE et al. 1982).

Bei den Mechanismen der Adhäsion von Bakterien auf der Zahnoberfläche handelt es sich in erster Linie um Protein- bzw. hydrophobe Bindungen. Die initiale Anlagerung von Bakterienzellen ist jedoch nicht nur chemisch bedingt, auch spielen mechanische Faktoren wie Unregelmäßigkeiten der Zahnoberfläche eine Rolle (PLAGMANN 1998). Findet zu diesem Zeitpunkt keine mechanische Plaquekontrolle statt, so verschiebt sich das bakterielle Verhältnis innerhalb der ersten zwei Tage hin zu einem größeren Anteil an gramnegativen Kokken. Hierbei handelt es sich im Wesentlichen um Veillonellen. Grampositive Kokken machen nun nur noch etwa die Hälfte der mikrobiellen Flora aus (THEILADE et al. 1982). Zunehmend treten grampositive und gramnegative Stäbchen auf.

Die Mikroorganismen beginnen mit der enzymatischen Spaltung niedermolekularer Kohlenhydrate; es entstehen organische Säuren, vorwiegend Laktat, welche ein azides Milieu verursachen. Gleichzeitig erfolgt der Aufbau der amorphen Plaquematrix durch die Synthese extrazellulärer Polysaccharide. Die extrazellulären Polysaccharide bestehen aus Glukanen und Fruktanen, diese werden durch die bakterieneigenen Enzyme Fruktosyl- und 
Glycosyltransferase aus Saccharose synthetisiert. Granulozyten und desquamierte Epithelzellen haften sich dem „Pellicle“ an. Entlang der Oberfläche dieser Epithelzellen kommt es zu einem Transport adhärenter Mikroorganismen zur Zahnoberfläche (THEILADE 1984). Die Plaque besteht zu diesem Zeitpunkt aus etwa 10-20 Zell-Lagen. Die auf der Zahnoberfläche lokalisierten Bakterien haften der Hartsubstanz noch eher lose an; ihr überwiegender Teil ist durch Wasserspray entfernbar.

\section{Phase 2 (ca. 3. - 7. Tag)}

Diese Phase ist gekennzeichnet durch weitere bakterielle Vermehrung und Dickenzunahme der Plaque; des Weiteren kommt es zu Veränderungen innerhalb der Plaquepopulation.

Wird weiterhin auf Mundhygienemaßnahmen verzichtet, so entwickelt sich zwischen dem dritten und siebten Tag eine Dominanz gramnegativer Bakterien in der Plaqueflora. Der weitere Plaqueaufbau vollzieht sich in verschiedenen Stadien. Fusobakterien und Filamente herrschen am dritten und vierten Tag vor (LISTGARTEN 1976), ab dem fünften Tag erfolgt die Einlagerung von Spirochäten und Spirillen. Der Anteil grampositiver Kokken und Stäbchen verringert sich von 90 auf etwa 50\% (PLAGMANN 1998).

Mit Hilfe des Elektronenmikroskopes ist in der nun etablierten Plaque eine typische Schichtung zu erkennen: Der Zahnoberfläche ist eine Schicht polymorpher Mikroorganismen aufgelagert, darüber sind Kokken angeordnet. Über der Kokkenschicht sind senkrecht zur Zahnoberfläche, meist palisadenartig angeordnete Stäbchen lokalisiert. Intermikrobiell findet man die Plaquematrix, welche zur Verklebung und Verfilzung der Bakterien führt. Zu diesem Zeitpunkt ist eine Entfernung des Biofilms nur noch im Rahmen einer Zahnreinigung, d.h. durch mechanische Plaquekontrolle, möglich.

\section{$\underline{\text { Phase } 3}$}

In der letzten Phase, zwischen dem 7. und 14. Tag, reift die Plaque weiter und gewinnt durch Teilungsvorgänge und Akkumulation weiterer Bakterien an Dicke (bis zu 0,5mm). In diesem Stadium besteht die Plaque aus bis zu 300 Zelllagen und weist eine deutliche histologische Struktur auf.

Da sich durch die steigende Plaquedicke die Lebensbedingungen für Anaerobier verbessern, gewinnt die Plaque zunehmend anaeroben Charakter. Ein $\mathrm{mm}^{3}$ Plaque (= 1mg Nassgewicht) enthält nun 100-300 Millionen Mikroorganismen (THEILADE 1984, RENGGLI 1990), womit die volle Säurebildungspotenz der Plaque erreicht ist. Die bakterielle Zusammensetzung kann, je nach oraler Entnahmestelle, stark variieren (BOWDEN et al. 1978). In den unteren 
Plaqueschichten, nahe der Zahnoberfläche, wächst die Anzahl gramnegativer Stäbchen. Die für ihr Wachstum erforderlichen Wachstumsfaktoren sind zwar im Speichel nicht verfügbar, werden jedoch durch das entzündlich bedingt vermehrt gebildete Sulkusfluid bereitgestellt. Eine zusätzliche Optimierung des Nährstoffangebotes erfolgt durch mikrobielle Symbiosen sowie durch Tod und Zerfall von Bakerienzellen (THEILADE 1984).

Stehen niedermolekulare Kohlenhydrate in ausreichender Menge zur Verfügung, so synthetisieren die Bakterien - quasi als Abwehr gegen die für sie lebensbedrohliche Überzuckerung extrazelluläre Polysaccharide (KÖNIG 1987, RENGGLI 1990). Diese werden für den Aufbau der organischen Plaquematrix genutzt und sind somit für die Kohäsion des Biofilms von großer Bedeutung. Das für die Plaque charakteristische Streptokokkenglukan wird vorwiegend von S. mutans gebildet und daher auch als Mutan bezeichnet. Es ist wasserunlöslich und sorgt daher für einen besonders soliden Verbund zwischen dem Biofilm und seiner Unterlage (KÖNIG 1987). Man spricht nun von ausgereifter, voll pathogener Plaque.

\section{2 Ätiologie der Karies}

Die häufigste Erkrankung der Zahnhartsubstanzen ist die Karies. Die Entstehung der Karies ist ein multifaktorieller, dynamischer Prozess; Phasen der Demineralisation und der Remineralisation wechseln sich ab. Karies führt zur Zerstörung von Zahnhartsubstanzen und schließlich zur Kavitation. Je nach Ausprägung äußert sie sich in unterschiedlicher Symptomatik. Die Symptome reichen von Veränderungen im Bereich der Gitterstruktur von Schmelzkristalliten über die mikroskopisch nachweisliche Zersetzung der Zahnhartsubstanzen bis hin zu klinisch sichtbaren Destruktionen und offenen kariösen Läsionen. Karies kann durch Phasen der Stagnation, Remission und Progression gekennzeichnet sein (HELLWIG et al. 1999).

Erfolgt keine adäquate Therapie eines bestehenden kariösen Defektes, so schreitet das Krankheitsbild voran. Ohne invasive Therapie führt dies schließlich zum Zahnverlust. Nur im Falle minimaler Demineralisationen kann von einer invasiven Therapie Abstand genommen werden. Hier können angemessene prophylaktische Maßnahmen eine Progression des Krankheitsbildes verhindern. In einzelnen Fällen kann von einer vollkommenen Regeneration des Zahnhartgewebes durch Remineralisationsvorgänge ausgegangen werden.

Bereits im 19. Jahrhundert setzte sich der US-amerikanische Zahnarzt Willoughby D. Miller (1853 - 1907) mit der Rolle der Mikroorganismen der Mundhöhle bei der Kariesentstehung auseinander. Seine Theorie eines „chemoparasitären Vorgangs“ (MILLER 1889) bildet die 
Grundlage der heute gültigen Theorie der Kariesentstehung, obwohl sie bis weit ins 20. Jahrhundert hinein nicht anerkannt wurde. Die chemoparasitäre Theorie besagt, dass kariogene orale Mikroorganismen, wie man sie in der dentalen Plaque findet, bei der Zufuhr kariogenen Substrats (niedermolekulare Kohlenhydrate) organische Säuren produzieren.

Erst über 70 Jahre nach Millers chemoparasitärer Theorie entwickelte KEYES ein Modell der Kariesätiologie. Mit Hilfe von Experimenten an Nagetieren stellte er heraus, dass Karies durch das Zusammenwirken der Faktoren „Zahn“, „Mikroorganismen“ und „Substrat für die Mikroorganismen“ entsteht (KEYES 1960 und 1962). 1987 ergänzte KÖNIG diese Darstellung des Kariesmodells durch den Faktor „Zeit“, welcher wie die drei bereits erwähnten Faktoren maßgeblich an der Kariesentstehung beteiligt ist. Demnach findet eine Zerstörung der Zahnhartsubstanzen erst infolge lang anhaltender bzw. häufiger Säureangriffe statt. Die primären ätiologischen Faktoren, die zur Entstehung einer Karies führen, sind demzufolge Zahn (Wirt), bakterieller Zahnbelag (Plaque), die Aufnahme niedermolekularer Kohlenhydrate (Substrat) und die (Einwirk-) Zeit.

Bei den für die Kariogenese bedeutsamen Mikroorganismen handelt es sich um verschiedene Spezies der Mutans-Streptokokken. Humanpathogene Arten sind S. mutans, S. sobrinus, S. cricetus und S. rattus. Die beiden erstgenannten findet man in der kariogenen Plaque am häufigsten vor (HELLWIG et al. 1999).

Mikroorganismen und Substrat können allein noch keine Karies verursachen. Die Bakterien müssen immer in Form von Plaque, eines organisch strukturierten Belages, auf den Zahnoberflächen vorhanden sein. Verschiedene Studien zeigten, dass Karies ohne Plaque nicht in Erscheinung tritt (ORLAND et al.1954 und 1955, AXELSSON und LINDHE 1978).

Neben den oben genannten Hauptfaktoren spielen allerdings auch verschiedene sekundäre Faktoren in der Kariesätiologie eine Rolle. Dazu zählen beispielweise die Zusammensetzung und Fließrate des Speichels, dessen pH-Wert und Pufferkapazität, die Dauer und Häufigkeit der Substrataufnahme sowie immunologische, genetische und sozioökonomische Faktoren. Außerdem können Stellungsanomalien der Zähne und Zahnfehlbildungen die Progression einer kariösen Läsion fördern (HELLWIG et al. 1999).

\section{$\underline{\text { Kariogenese }}$}

Das für die Plaquebakterien wichtigste Substrat stellen niedermolekulare Kohlenhydrate dar. Eine entscheidende Rolle in der Kariogenese spielen Mono- und Disaccharide. Diese wirken potentiell kariogen, da sie durch bakterielle Stoffwechselvorgänge zu organischen Säuren abgebaut werden. Zu den häufigsten Monosacchariden zählen Glukose, Fruktose, Galaktose 
und Mannose; wichtige Disaccharide sind Laktose, Maltose und Saccharose. Die Saccharose nimmt in dieser Reihe eine Sonderstellung ein, da sie für die Bakterien der Plaque vor allem für S. mutans, den Leitkeim der Karies, ein optimales Substrat darstellt: Durch extrazelluläre enzymatische Spaltung entstehen aus Saccharose Glukose und Fructose. Die Glukose nutzt das Bakterium zur Synthese klebriger extrazellulärer Polysaccharide. Diese Polysaccharide wiederum ermöglichen den Plaquebakterien eine zusätzliche Adhäsion an der Zahnoberfläche. Die beim Abbau der Saccharose entstandene Fruktose wird durch ein spezielles Transportsystem des Bakteriums (Phosphoenolpyruvat-Phosphotransferasesystem = PEP-PTS) in die Zelle eingeschleust und dort zur Produktion intrazellulärer Polysaccharide genutzt. Intrazelluläre Polysaccharide werden von den Mikroorganismen als Speicher- bzw. Reservekohlenhydrate verwendet. So sind sie in der Lage, ihren Metabolismus auch in Phasen verminderter Substratzufuhr aufrechtzuerhalten. In verschiedenen Studien wurde der Zusammenhang zwischen dem Konsum niedermolekularer Kohlenhydrate und der Kariesentstehung evaluiert: Die „Vipeholm-Studie“ (GUSTAFSSON et al. 1954) stellte heraus, dass das kariogene Potenzial von Kohlenhydraten nicht von der absoluten Menge der Zufuhr abhängig ist, sondern dass die Häufigkeit des Zuckerkonsums einen weitaus bedeutenderen Einfluss auf die Kariesentwicklung hat. Das Adhäsionsvermögen einer kohlenhydrathaltigen Speise spielt ebenfalls eine große Rolle, da dieses die Verweildauer der Speise im Mund bestimmt. KÖNIG und MÜHLEMANN erwiesen 1967, dass das kariogene Potenzial des Zuckers zunimmt, je feinkörniger und klebriger er ist. Die Turku-Zucker-Studien (SCHEININ und MÄKINEN 1975) ergaben, dass ein Austausch des Zuckers durch den Zuckeraustauschstoff Xylit die Entstehung kariöser Läsionen nahezu verhindert. S. mutans verfügt aber nicht nur über die Fähigkeit, intra- und extrazelluläre Polysaccharide zu synthetisieren, sondern besitzt zusätzlich azidogenes Potenzial: Das Bakterium spaltet das Disaccharid Saccharose enzymatisch. Die dabei freiwerdende Glukose wird in die anaerobe Glykolyse eingeschleust, und es erfolgt die Bildung organischer Säuren (Laktat). Milchsäure ist die stärkste organische Säure und führt nach Säureattacken auf den Zahnschmelz zur irreversiblen Demineralisation der Zahnhartgewebe (STEPHAN 1944, HEINRICH-WELTZIEN 1998). Calcium und Phosphat werden solange aus dem Zahnschmelz herausgelöst, wie ausreichende Mengen von Säure produziert werden. Der „kritische pH-Wert“ für Schmelz liegt bei etwa 5,5; in Dentin und Wurzelzement finden erste Demineralisationsvorgänge bereits ab einem pH-Wert von 6,5 statt. Eine weitere Besonderheit von S. mutans ist, dass das Bakterium azide, mikroökologische Bedingungen toleriert. S. mutans besitzt die Fähigkeit, Säuren gegen den Konzentrationsgradienten aus dem Zell-inneren auszuschleusen (HELLWIG et al. 1999). 
Weitere in der oralen Flora auftretende Mikroorganismen, denen eine wichtige Bedeutung bei der Pathogenese der Karies zukommt, sind Laktobazillen und Actinomyzeten. Hohe Laktobazillenwerte im Speichel können ein Indikator für offene kariöse Läsionen sein, in denen sie als säuretolerante Keime ein optimales Milieu und Nährstoffe finden und vor einer Ausspülung aus der Mundhöhle geschützt sind; analog sind sie in tiefen Fissuren anzutreffen (KNEIST 1998). Laktobazillen produzieren zwar weniger Milchsäure aus Kohlenhydraten der Nahrung als Mutans-Streptokokken, sind aber stärker azidurisch und können MutansStreptokokken bei sinkendem pH-Wert in Kavitäten und Fissuren überleben und weiter Säure bilden. Aktinomyzeten hingegen sind relativ schwach azidogen, sie werden hauptsächlich mit der Entstehung der Wurzelkaries assoziiert.

Zusammenfassend lässt sich feststellen:

1. Säurebildung und -toleranz sowie die Synthese intra- und extrazellulärer Polysaccharide sind die bedeutendsten Eigenschaften kariogener Keime.

2. Bei der Karies handelt es sich nicht um eine spezifische Infektion; die alleinige Infektion mit S. mutans führt nicht zur Entstehung einer Karies.

3. Das kariogene Potenzial der Bakterien ist abhängig von der Zufuhr adäquaten Substrats.

4. Wirtsfaktoren (z.B. Speichel) können die kariogene Wirkung der Mikroorganismen beeinflussen.

\section{3 Ätiologie der entzündlichen Parodontopathien}

Je nach Ausprägungsart ist die Parodontitis eine mehr oder weniger generalisierte entzündliche multifaktorielle Erkrankung des Zahnhalteapparates (RATEITSCHAK et al. 2004). Bakterielle Anlagerungen an Zahn und Mundschleimhaut in Form eines Biofilms bedingen marginale Entzündungsreaktionen, welche bei der Präsenz von Risikofaktoren, reduziertem Immunstatus, inflammatorischen Mediatoren und bei gehäuftem Auftreten parodontopathogener Keime auf tiefere Strukturen des Zahnhalteapparats übergreifen und eine Destruktion desmodontaler Fasern sowie des Knochens hervorrufen (RATEITSCHAK et al. 2004). Es resultiert die Ausbildung parodontaler Taschen; fortschreitender Knochenabbau kann zur Zahnlockerung und schließlich zum Zahnverlust führen (SCHROEDER 1996).

Bereits 1965 konnten LÖE und dessen Mitarbeiter einen Zusammenhang zwischen entzündlichen Parodontalerkrankungen und bakterieller Plaque nachweisen (LÖE et al. 1965). Im Rahmen der „experimentellen Gingivitis“ erbrachte er den klassischen experimentellen Be- 
weis dafür, dass bakterielle Zahnbeläge die Entwicklung einer Gingivitis provozieren: Wird bei plaque- und gingivitisfreien Verhältnissen auf Mundhygienemaßnahmen verzichtet, so kommt es zur Plaqueakkumulation. Innerhalb weniger Tage resultiert dann immer eine leichte Gingivitis. Bei Plaqueentfernung und Wiederaufnahme der Mundhygiene bildet sich die Gingivitis jedoch zurück. Gingivitis ist demnach reversibel, es erfolgt eine Restitutio ad integrum. Während bakterielle Zahnbeläge immer zur Etablierung einer entzündlichen Gingivitis führen, muss eine Akkumulation dentaler Plaque nicht zwangsläufig die Entstehung einer Parodontitis verursachen. Bei vergleichbarer Plaquemenge reicht das klinische Bild von vollkommen gesunden gingivalen Verhältnissen über leichte Entzündungszeichen bis hin zur schweren Parodontitis mit massiven Knochendestruktionen (GRIFFITH et al. 1988, SEYMOUR 1991).

Eine etablierte, plaque-induzierte Gingivitis kann ohne weitere Konsequenzen jahrelang stabil bleiben, sie kann jedoch auch in eine Parodontitis übergehen. Welche Faktoren den entscheidenden Abbau der tieferen parodontalen Gewebe auslösen, der schließlich zur fortgeschrittenen Parodontitis führt, ist nicht allgemeingültig definierbar (RENGGLI 1990). Im Gegensatz zur Karies ist es bei der Parodontitis nicht möglich, einen speziellen Erreger als Leitkeim zu bestimmen. Bei den entzündlich bedingten Parodontitiden führt die Anwesenheit verschiedener gramnegativer Mikroorganismen in Kombination zur Krankheitsentstehung. Dazu gehören insbesondere Actinobacter actinomycetemcomitans, Prevotella intermedia, Porphyromonas gingivalis, Tannerella forsythia, Tannerella denticula und Spirochäten (DAHLE et al. 1993, WOLFF et al. 1994, LANG 2003, RATEITSCHAK et al. 2004). Die Gingivitis ist eher auf die Menge und die Metaboliten der Plaque zurückzuführen als auf spezifische Keime (RATEITSCHAK et al. 2004).

Die Entwicklung einer Parodontitis auf der Basis einer Gingivitis verläuft folgendermaßen: Mit der Progredienz der Entzündung lockert sich das gingivale Gewebe durch bakterielle Toxine und entzündlich bedingte Reaktionen auf, sodass sich der Biofilm auch in subgingivale Bereiche ausbreiten kann. Durch Proliferation und apikalwärts gerichtete Ausdehnung des Biofilms entsteht eine echte parodontale Tasche. Während das entzündliche Exsudat bei der Gingivitis mit noch annähernd normal tiefem Sulkus in Richtung Plaque eher inzisalwärts strömt, erfolgt bei der Parodontitis eine Richtungsänderung des Exsudatstromes: Er fließt nun vom Infiltrat her kommend senkrecht in Richtung der plaquebesiedelten Zahnoberfläche. Der Epithelansatz verschiebt sich nach apikal. Durch Plaqueentfernung kann nun, anders als bei der Gingivitis, keine Restitutio ad integrum mehr erreicht werden, jedoch kommt es nach Wurzelreinigung zu Regenerationen (RATEITSCHAK et al. 2004). 


\subsection{Bedeutung der Prophylaxe}

Die Mundhygiene zählt neben Ernährungslenkung, Fluoridapplikation und Fissurenversiegelung zu den zentralen Pfeilern der zahnmedizinischen Prophylaxe (LEHMANN und HELLWIG 1998, HELLWIG et al. 1999). Plaquekontrolle in Form einer regelmäßigen und möglichst vollständigen Elimination des bakteriellen Biofilms stellt die effektivste Form der Prävention von Karies, Gingivitis und Parodontitis dar (KEYES 1960 und 1962, AXELSSON und LINDHE 1978, ROULET 1995). Bereits 1965 zeigten LÖE et al. mit der experimentellen Gingivitis den Zusammenhang zwischen bakterieller Plaque und entzündlichen Veränderungen der Gingiva auf (Vgl. Kapitel 2.3).

Voraussetzung für den dauerhaften Erhalt gesunder dentaler und parodontaler Verhältnisse ist daher das Erlernen einer adäquaten Mundhygiene. Oralhygienische Maßnahmen und die Intensivierung der häuslichen Mundhygiene bilden die Grundlage für eine effektive Plaquekontrolle.

Man unterscheidet die mechanische von der chemischen Plaquekontrolle. Hilfsmittel der mechanischen Plaquekontrolle sind Zahnbürste, Zahnseide bzw. Superfloss, Interdentalraumbürstchen, Zahnpasta und Zungenschaber. Für den Erhalt der Zahngesundheit spielt die chemische Plaquekontrolle eine eher untergeordnete Rolle (RATEITSCHAK et al. 2004).

Die Zahnreinigung mit Hilfe der Zahnbürste hat sich in fast allen zivilisierten Ländern als effektivste Methode der Plaqueentfernung bewährt (FRANDSEN 1985). 1976 fanden SCHMID et al. heraus, dass durch den Gebrauch der Zahnbürste bis zu 86\% des Zahnbelages auf oralen und bukkalen Zahnoberflächen und an erreichbaren approximalen Flächen bis zu 74\% beseitigt werden können. Durch die zusätzliche Anwendung interdentaler Mundhygienehilfsmittel wurden maximal $96 \%$ des Biofilms aller Zahnoberflächen eliminiert. Plaquekontrolle mit Hilfe einer Zahnbürste ermöglicht folglich eine effektive Entfernung von Zahnbelägen, führt jedoch selten zu vollkommener Plaquefreiheit (FRANDSEN et al. 1986). Zur Prävention der Karies und der plaque-induzierten Gingivitiden kommt der korrekten Anwendung der Zahnbürste eine besonders große Bedeutung zu. Mangelnde Kenntnisse über effiziente Reinigungstechniken und, angesichts einer immer älter werdenden Bevölkerung, zunehmend auch eingeschränkte manuelle Fähigkeiten beeinträchtigen die praktische Umsetzung. Als Hauptursache dafür, dass durch den Gebrauch der Zahnbürste keine vollständige Belagsentfernung erzielt wird, wird zudem eine unzureichende Putzdauer diskutiert (VAN 
DER WEIJDEN et al. 1993, VAN DER WEIJDEN et al. 1996a). Verschiedene Studien zeigen, dass sowohl bei der Verwendung von Handzahnbürsten als auch beim Gebrauch elektrischer Zahnbürsten die Plaquereduktion mit zunehmender Putzdauer ansteigt (MC CRACKEN et al. 2003, VAN DER WEIJDEN et al. 1993, WILLIAMS et al. 2004). Dass längere Putzzeiten nicht zwingend zu einer effektiveren Plaqueentfernung habituell unsauberer Bereiche führen, belegen andere Studien: So ist die Reinigung der Bukkalflächen signifikant gründlicher als die der Oralflächen, die der Lingualflächen des Unterkiefers schlechter als die der Palatinalflächen des Oberkiefers und die Reinigung der Frontzähne und Prämolaren besser als diejenige im Molarenbereich (HUBER et al. 1985, HAWKINS et al. 1986). FRANDSEN et al. stellen heraus, dass eine eineinhalbminütige Zahnreinigung, unabhängig von der angewandten Technik, nicht zu vollkommener Plaquefreiheit führt (FRANDSEN et al. 1970). Eine andere Studie zeigte ähnliche Ergebnisse: Während einminütiges Putzen für eine zufriedenstellende Plaquereduktion nicht ausreichte, konnte die Plaque durch Verdoppeln der Putzdauer signifikant reduziert werden. Allerdings resultierte die Erhöhung der Putzdauer auf drei bzw. vier Minuten nicht in einer signifikant gesteigerten Plaquereduktion gegenüber der zweiminütigen Zahnreinigung (VAN DER WEIJDEN et al. 1993 und 1996a). Laut einer epidemiologischen Untersuchung putzen 75\% der Deutschen ihre Zähne kürzer als 90 Sekunden bzw. weniger als zweimal täglich (BAUCH et al. 1991). Angesichts der hier ermittelten Putzgewohnheiten scheint die Forderung der Präventivmedizin, die Zähne nach jeder Mahlzeit zu reinigen sowie eine tägliche Reinigung der Interdentalräume durchzuführen (MINTEL und CRAWFORD 1992, WARREN PR et al. 1998) auf einen Großteil der deutschen Bevölkerung nicht übertragbar zu sein.

Eine Steigerung des Mundhygieneniveaus allein durch Motivation und Instruktion zu erzielen, scheint äußert schwierig. Ob es durch neu entwickelte, elektrische Zahnbürsten gelingt, die Effektivität hinsichtlich der Plaquereduktion so zu steigern, dass bei gleich bleibender Putzdauer eine gründlichere Reinigung erzielt wird, ist fraglich.

\subsection{Prophylaxeinstrument Zahnbürste}

\subsubsection{Historischer Hintergrund}

Wie archäologische Funde in altägyptischen Gräbern belegen, wurden zahnbürstenähnliche Geräte für die Reinigung der Zähne bereits vor etwa 5000 Jahren eingesetzt. Hierbei handelte es sich um Zweige des sogenannten Miswak („Zahnbürstenbaum“). Von den Enden dieser 
Zweige wurde die Rinde entfernt; anschließend wurden die Enden solange gekaut, bis sie zerfaserten und ein pinselähnliches Mundhygieneinstrument entstand. Um 1500 nach Christus entwickelte man in China die ersten bürstenähnlichen Zahnpflegehilfsmittel; diese bestanden aus Borsten von Hausschweinen, die an einem Griff aus Bambus oder Knochen befestigt waren (SCHWEISHEIMER 1969). Im deutschen Sprachraum fand die Zahnbürste erstmals um 1700 Erwähnung, vorher wurden die Zähne überwiegend mit Schwämmen und Läppchen gereinigt. Der Engländer William Addis gründete im Jahr 1780 die erste Fabrik, die Zahnbürsten aus Kuhknochen und -borsten herstellte. Da die Produktion jedoch sehr aufwändig und teuer war, galten Zahnbürsten zu dieser Zeit eher als ein „Luxus der Wohlhabenden“. Zwar erwachte im 18. Jahrhundert, im Zeitalter der Aufklärung, ein neues Gesundheitsbewusstsein, jedoch galt die Zahnpflege selbst unter zahnheilkundlich orientierten Medizinern als umstritten. Die Überzeugung von der Notwendigkeit einer täglichen Zahnpflege verbreitete sich erst zu Beginn des 19. Jahrhunderts.

Erst 1938 ermöglichte die Erfindung des Nylons eine kostengünstige Massenherstellung von Zahnbürsten. Seither wurde die Zahnbürste in Design und Material kontinuierlich weiterentwickelt. Die erste elektrische Zahnbürste wurde 1960 in den USA vorgestellt.

\subsubsection{Handzahnbürsten}

Heute werden an eine optimale Handzahnbürste folgende Anforderungen gestellt (BASS 1948, SAUERWEIN 1962, HELLWIG et al. 1999):

- der Griff ist einfach und gerade,

- der Bürstenkopf ist kurz ( Länge: ca. 2,5 cm),

- das Borstenfeld ist plan,

- die Bürste besitzt gerade, elastische, an den Enden abgerundete Kunststoffborsten,

- die Borsten sind weich bis mittelhart,

- die Borstenlänge beträgt 10-12mm,

- die Dicke der einzelnen Borste beträgt 0,18-0,25mm,

- das Borstenfeld setzt sich aus Büscheln von je 20-40 Borsten zusammen („multi-tufted“).

Bis in die erste Hälfte des zwanzigsten Jahrhunderts dauerte die Vorherrschaft traditioneller Naturborsten an. Bei diesen Borsten handelte es sich in der Mehrzahl um Nackenborsten von Hausschweinen. Naturborsten weisen jedoch zwei gravierende Nachteile auf: Zum einen nehmen sie vermehrt Wasser auf, wodurch sie aufweichen und folglich schneller verschleißen, zum anderen weisen Naturborsten Hohlräume auf, in denen sich Bakterien ansammeln 
und vermehren können (LANGE 1986). Naturborsten wurden zunächst durch Zelluloid, ab 1948 jedoch hauptsächlich durch Nylon-Filamente verdrängt.

BASS forderte abgerundete Borstenenden, um durch das Zähneputzen verursachte Gingivaverletzungen zu vermeiden (BASS 1948). Ein Abrunden der Borstenenden ist seit den 50er Jahren möglich und wurde seitdem mehrfach modifiziert. Heute sollen Zahnbürstenborsten eine geringe Wasseraufnahme und vollständig abgerundete Enden aufweisen (GOLDING 1982). Die Deutsche Industrienorm (DIN) 13917 definiert seit 1979 den Aufbau, die Gestalt und die Form von Handzahnbürsten.

\subsubsection{Elektrische Zahnbürsten}

Elektrische Zahnbürsten haben sich heute als Alternative zu konventionellen Handzahnbürsten fest etabliert. Unzureichende Compliance und Motivation sowie reduzierte manuelle Geschicklichkeit des Patienten führen bei der Verwendung traditioneller Zahnbürsten zur Etablierung vorwiegend horizontaler Schrubbbewegungen, welche keine akzeptable Plaquereduktion gewährleisten und als schädigend für die Zahnhartsubstanzen einzuschätzen sind. Nicht nur bei Patienten mit eingeschränkter manueller Geschicklichkeit wie beispielsweise Kindern, geistig oder körperlich Behinderten und Senioren stellen elektrische Zahnbürsten aufgrund ihrer einfacheren Handhabung eine geeignete Alternative dar. Verschiedene Untersuchungen zeigen nicht nur Verbesserungen der Mundhygiene bei Behinderten unter Verwendung elektrischer Zahnbürsten (BRATEL et al.1988, BRATEL und BERGGREN 1991), sondern belegen auch eine effektivere Plaqueentfernung im Vergleich zu manuellen Zahnbürsten bei Kindern (GROSSMANN und PROSKIN 1997, JONGENELIS und WIEDEMANN 1997). Die kontinuierliche Weiterentwicklung der elektrischen Zahnbürsten konnte jedoch dazu beitragen, dass diese Geräte nicht mehr ausschließlich geistig oder körperlich beeinträchtigten Patienten bzw. Patienten während kieferorthopädischer Behandlung empfohlen werden; elektrische Zahnbürsten sind heutzutage vielmehr als wichtiger Baustein der modernen Oralprophylaxe zu betrachten. Gemäß ihrer Bürstenkopfbewegungen können sie in drei Generationen eingeteilt werden (ZIMMER et al. 1999):

Die in den frühen 60er Jahren entwickelte erste Generation elektrischer Zahnbürsten besitzt einen länglichen Bürstenkopf und führt Schwenk- und Seitwärtsbewegungen aus. Geräte der ersten Generation haben auf dem heutigen Markt keinen Stellenwert mehr.

Die zweite Generation elektrischer Zahnbürsten wurde 1987 eingeführt. Hierbei handelt es sich um rotierend-oszillierende Bürstensysteme, die durch runde Bürstenköpfe gekennzeichnet sind. Geräte dieser Generation basieren auf einfachen Elektromotoren, deren Antrieb über 
Zahnräder oder exzentrische Wellen erfolgt. Ähnlich eines rotierenden Polierkelchs im zahnärztlichen Winkelstück werden Zahnbeläge bei diesem Zahnbürstentyp durch direkten Kontakt der Bürste zur Zahnoberfläche entfernt (VAN DER WEIJDEN et al. 1994, DENTINO et al. 2002, SICILIA et al. 2002). Die neuesten Modelle der rotierend-oszillierenden Zahnbürsten generieren dreidimensionale Bürstenkopfbewegungen, indem sie Rotationen und Pulsationen kombinieren. Rotierend-oszillierende Bürstensysteme stellen das größte Marktsegment dar.

1993 wurde erstmals die dritte Generation elektrischer Zahnbürsten vorgestellt. Zu dieser Gruppe zählen sogenannte schallaktive Bürsten mit länglichem Bürstenkopf, deren Borsten mit Frequenzen zwischen 250 und 300 Hertz oszillieren. Schallaktive Zahnbürsten verfügen über magneto-elektrische Motoren, deren Antrieb über den piezo-elektrischen Effekt erfolgt. Elektrische Zahnbürsten der dritten Generation stellen ein kontinuierlich wachsendes Marktsegment dar.

\subsubsection{Elektrisch unterstützte Handzahnbürsten}

Elektrisch unterstützte Handzahnbürsten sind seit 2007 auf dem Markt. Sie sind batteriebetrieben und haben, bei zweimaliger, zweiminütiger Anwendung pro Tag, eine Lebensdauer von etwa drei Monaten. Die Putztechnik entspricht derjenigen der konventionellen Handzahnbürsten. Die batteriebetriebenen manuellen Zahnbürsten generieren zusätzlich Vibrationen der Borsten, die die Plaqueentfernung positiv beeinflussen sollen.

\subsection{Effektivität elektrischer Zahnbürsten im Vergleich zu Handzahnbürsten}

Um die Effektivität manueller und elektrischer Zahnbürsten miteinander zu vergleichen, wurden zahlreiche wissenschaftliche Untersuchungen durchgeführt. Bereits in den Sechziger Jahren war man sich darüber einig, dass elektrische Zahnbürsten hinsichtlich der Reinigungseffektivität mit den Handzahnbürsten konkurrieren konnten (QUIGLEY und HEIN 1962, ASH 1964). Zu dieser Zeit ging man besonders im amerikanischen Raum nicht nur davon aus, dass die elektrische Zahnbürste konkurrenzfähig sei, sondern stellte sogar ihre Überlegenheit gegenüber der Handzahnbürste heraus (LEFKOWITZ und ROBINSON 1962, POWERS et al. 1967). Rotierend-oszillierenden Bürstensystemen wird hinsichtlich der Plaque- und Gingivitisreduktion bis heute eine signifikant höhere Effektivität bescheinigt. LAZARESCU et al. untersuchten 2003 die Effektivität der Plaquereduktion bzw. den individuellen „Lerneffekt“ einer elektrischen gegenüber einer manuellen Zahnbürste. Achtzig Studienteilnehmer, 
die bisher keinerlei Erfahrung im Umgang mit elektrischen Zahnbürsten hatten, wurden hierfür in zwei Gruppen zu je 40 Probanden unterteilt. Probanden der Gruppe I verwendeten über einen Zeitraum von 18 Wochen eine elektrische Zahnbürste, während in Gruppe II mit einer Handzahnbürste geputzt wurde. Kontrolltermine erfolgten nach jeweils drei, sechs, zwölf und 18 Wochen; jede Evaluation umfasste zwei Kontrolltermine: An Tag 1 wurden ein Plaqueund ein Gingivaindex erhoben. Anschließend stellten die Probanden über 24 Stunden jegliche Mundhygienemaßnahmen ein. An Tag zwei wurde der Plaqueindex (PI) erneut erhoben, darauf folgte ein dreiminütiges überwachtes Putzen, um im Anschluss daran wiederum den PI zu ermitteln. Der „Lerneffekt“ wurde durch die prozentuale Plaqueentfernung nach der überwachten Zahnreinigung ausgedrückt. Dieser war bei den Teilnehmern, welche die elektrische Zahnbürste verwendeten, signifikant besser als bei den Probanden, die die Handzahnbürste benutzten. Die Unterschiede zwischen beiden Gruppen hinsichtlich der Plaque- und Gingivitisreduktion waren ebenfalls statistisch signifikant. Sowohl der Plaque- als auch der Gingivaindex verringerte sich in Gruppe I deutlicher als in Gruppe II (LAZARESCU et al. 2003).

Eine Sichtung von 354 Studien zum Thema Effektivität elektrischer Zahnbürsten im Vergleich zu Handzahnbürsten erfolgte durch das Cochrane-Institut (COCHRANE Database 2003). Es wurden nur randomisierte kontrollierte Studien mit einer Untersuchungsdauer von mindestens 28 Tagen in die Metaanalyse eingeschlossen, ausgewertet werden konnten letztendlich 29 Studien. Die Ergebnisse zeigten, dass elektrische Zahnbürsten Plaque und Gingivitis effizienter reduzierten als manuelle Zahnbürsten. Im Jahr 2005 wurde die CochraneUntersuchung unter Einschluss 13 weiterer Studien aktualisiert; die Ergebnisse blieben jedoch gleich (COCHRANE Database 2005): Sowohl rotierend-oszillierende als auch schallaktive Geräte führten im Vergleich zu Handzahnbürsten zu einer statistisch signifikant höheren Plaque- und Gingivitisreduktion (HIMMER und EICKHOLZ 2008).

\subsection{Effektivität rotierend-oszillierender Zahnbürsten im Vergleich mit Schall- zahnbürsten}

Während der größte Teil elektrischer Zahnbürsten den Biofilm mechanisch, d.h. nur unter direktem Kontakt der Bürste zur Zahnoberfläche ablöst, sollen Schallzahnbürsten in der Lage sein, dynamische Strömungsaktivitäten zu erzeugen, welche dentale Plaque auch ohne unmittelbaren Kontakt der Borsten zum Zahn zerstören (ENGEL et al. 1993, JOHNSON und McINNES 1994). Ähnlich der Funktionsweise eines Ultraschall-Scalers, der beispielsweise 
zur Zahnsteinentfernung eingesetzt wird, kommt es durch Schallenergie zu einer raschen Volumenänderung der Gasbläschen im Wasser-Luft-Gemisch. So entstehen lokale Scherkräfte, die die Zahnoberfläche von der Plaque befreien (KHAMBAY und WALMSLEY 1995, STANFORD et al. 1997, HOPE und WILSON 2003). STANFORD stellte in seiner In-vitroUntersuchung heraus, dass Schallzahnbürsten den Biofilm aus einer Entfernung von bis zu 3 mm von der Zahnoberfläche ablösen können (STANFORD et al. 1997). Eine weitere Studie ergab, dass approximale Bereiche unter Verwendung einer Schallzahnbürste besser gereinigt werden als durch rotierend-oszillierende Bürstensysteme (YANKELL et al. 1997). Einige Invivo-Untersuchungen zeigten allerdings andere Ergebnisse. Hinsichtlich der Effektivität konnten hier keine signifikanten Unterschiede zwischen den beiden Zahnbürstentypen festgestellt werden (MORAN et al. 1995). Probanden favorisierten jedoch klar die rotierendoszillierenden Geräte (MORAN et al. 1995, BADER und BOYD 1999, VAN DER WEIJDEN et al. 1996b). In aktuelleren Untersuchungen resultierte der Effektivitätsvergleich von rotierend-oszillierenden und schallaktiven Zahnbürsten in einer höheren Plaque- und Gingivitisreduktion bei den Schallzahnbürsten. SCHAEKEN et al. fanden 2007, dass schallaktive Zahnbürsten supragingivale Plaque besser entfernten als rotierend-oszillierende. HOLT et al. untersuchten die Fähigkeit beider Zahnbürstentypen, Gingivitiden zu reduzieren. Eine Überlegenheit der Schallzahnbürste stellte sich hierbei lediglich für den Interdentalbereich heraus (HOLT et al. 2007).

Aus den Resultaten der bisher zitierten Studien kann keine Überlegenheit der einen gegenüber der anderen elektrischen Zahnbürste herausgestellt werden. Hierzu werden noch weitere klinische Untersuchungen erforderlich sein.

\subsection{Wann sollte eine Zahnbürste ausgewechselt werden?}

Die Zahnbürste ist ein täglich verwendeter Gebrauchsgegenstand mit begrenzter Lebensdauer. Sie unterliegt gewissen Verschleißerscheinungen, weshalb sie regelmäßig ausgetauscht werden sollte. Der regelmäßige Austausch der Zahnbürste stellt einen wichtigen Faktor für deren richtige Anwendung dar. Während im Allgemeinen nach etwa drei Monaten zu einem Zahnbürstenwechsel geraten wird, wird in der Literatur über dieses Anwendungsintervall und die Korrelation von Zahnbürstenabnutzung und Reinigungswirkung kontrovers diskutiert; wissenschaftliche Grundlagen für einen Nutzungszeitraum von drei Monaten finden sich fast keine (KOCH et al. 2007). Verschiedene individuelle Faktoren wie Putztechnik, Putzdauer und aufgewendete Putzkraft beeinflussen den jeweiligen Abnutzungsstatus einer Zahnbürste (Mc KENDRICK et al. 1971, KREIFELDT et al. 1980, DEAN 1991, DEAN et al. 1992). Die Be- 
antwortung der Frage nach der exakten Lebensdauer einer Zahnbürste wird dadurch erschwert.

Während einige Autoren einen Zahnbürstenwechsel aus hygienischen Gründen unabhängig vom Zustand der Zahnbürste nach spätestens sechs bis acht Wochen empfehlen (MÜLLER et al. 1989, GÜLZOW 1995, KLIMM 1997), ersetzt ein Großteil der Bevölkerung seine Zahnbürste erst dann durch eine neue, wenn Verschleißerscheinungen wie beispielsweise deformierte Borsten deutlich erkennbar sind (GLAZE and WADE 1986). VAN NÜSS et al. bewerteten in ihrer Untersuchung den Zustand abgenutzter Zahnbürstenborsten nach einer Gebrauchsdauer von drei bzw. sechs Monaten. Unter den 140 untersuchten Objekten befanden sich sieben verschiedene Erwachsenenzahnbürsten aus drei unterschiedlichen Preiskategorien, von denen 70 Zahnbürsten über drei Monate und 70 Bürsten über sechs Monate verwendet worden waren. Zwar wiesen die Borstenfelder aller getesteten Zahnbürsten Flächenzunahmen durch Borstendeformierungen auf, jedoch ergaben die makroskopische und lichtmikroskopische Untersuchung keine wesentlichen Unterschiede zwischen drei- und sechsmonatiger Gebrauchsdauer. Die Autoren diskutierten, ob eine ausgeprägte Flächenzunahme des Borstenfeldes zwangsläufig eine Verminderung der Borstenqualität bedingt und kamen zu dem Ergebnis, dass aus dem Ausmaß der Flächenzunahme keine Rückschlüsse auf den Zustand der Borsten gezogen werden können (VAN NÜSS et al. 2010).

Eine im Jahr 2005 erhobene unabhängige Umfrage des Marktforschungsinstituts NIELSEN ergab, dass die Deutschen ihre Zahnbürste lediglich 1,3-mal pro Jahr auswechseln; dies entspricht einer Gebrauchsdauer von nahezu einem Jahr. Auch andere Autoren fanden heraus, dass Zahnbürsten für gewöhnlich erheblich länger als drei Monate verwendet werden (DEAN et al 1992). Beim Vergleich der Mundgesundheit zwischen Deutschland und der Schweiz stellten STAEHLE und KERSCHBAUM fest, dass der jährliche Zahnbürstenverbrauch durchschnittlich bei zwei Bürsten pro Person liegt. Während die durchschnittliche Gebrauchsdauer von Handzahnbürsten somit sechs Monate beträgt, werden Bürstenköpfe elektrischer Zahnbürsten wesentlich seltener ausgewechselt; hier werden jährlich lediglich 0,25 Aufsteckbürsten pro Kopf verbraucht (STAEHLE und KERSCHBAUM 2003).

Untersuchungen, in denen die Studienteilnehmer selbst einschätzen sollten, wann sie ihre gebrauchte gegen eine neue Zahnbürste austauschen, zeigten hingegen eine deutlich kürzere Gebrauchsdauer: ZIEBOLZ zeigte mit einer Studie aus dem Jahr 2006, dass 75\% der Befragten ihre Zahnbürste nach eigenen Angaben nicht länger als drei Monate verwendeten. Nur 6\% der Teilnehmer gaben an, länger als sechs Monate mit derselben Zahnbürste zu putzen. Zu einem vergleichbaren Ergebnis kam auch GUNST (1999): Hier äußerten über 80\% der Pro- 
banden, ihre Zahnbürste nicht länger als drei Monate zu verwenden. Hierbei ist jedoch fraglich, inwiefern die tatsächliche mit der angegebenen Verwendungsdauer der Zahnbürsten übereinstimmt.

Nach KOCH et al. (2007) existieren drei Kriterien, die einen Zahnbürstenwechsel rechtfertigen:

1. Ineffizienz bei der Plaquereduktion

2. Mikrobielle Besiedlung der Borsten

3. Potentielle Verletzungsgefahr durch abgenutzte Borsten.

\subsubsection{Ineffizienz abgenutzter Borsten bei der Plaquereduktion}

Einem abgenutzten Borstenfeld wird die Fähigkeit abgesprochen, den oralen Biofilm in Furchen, Nischen und Spalten zu erreichen beziehungsweise zu eliminieren. Die Reinigungseffektivität einer Zahnbürste ist immer dann am besten, wenn die Borsten bei der Bürstenbewegung einem Richtungswechsel unterliegen (KOCH et al. 2007). Durch die Ausführung kleiner Rüttelbewegungen wie etwa bei der modifizierten Bass-Technik (BASS 1954) gelangen die elastischen Borsten auch in schwer zugängliche Bereiche wie den Interdentalraum und den gingivalen Sulkus (RATEITSCHAK et al. 2004). Eine adäquate Plaqueentfernung im Bereich des Sulkuseingangs sowie im Approximalraum ist unter der Verwendung abgenutzter, nicht mehr elastischer Borsten nicht zu erzielen. Folglich müsste die Reinigungswirkung einer abgenutzten Zahnbürste dort deutlich reduziert sein, weshalb ein Zahnbürstenwechsel angeraten wird, bevor die Borsten aufgebogen sind.

Klinische Studien mit künstlich abgenutzten Borsten stellten eine weniger effektive Plaqueentfernung im Vergleich zu neuen Zahnbürsten heraus (WARREN PR et al. 2002, $\mathrm{KOCH}$ et al. 2007). Einige Autoren erkannten, dass abgenutzte Zahnbürsten eine deutlich geringere Reinigungseffektivität besitzen als weniger abgenutzte. Der Abnutzungsstatus der untersuchten Zahnbürsten beeinflusste die Reinigungsfähigkeit, wobei die Plaquereduktion umso unzureichender wurde, je schwieriger die zu reinigenden Regionen zu erreichen waren. Nach Ansicht der Autoren könnten sowohl die Borstenermüdung als auch das mit zunehmender Gebrauchsdauer auftretende Verkürzen der Borsten zu einer solchen Verschlechterung führen (KREIFELDT et al. 1980).

DOHERTY et al. untersuchten ebenfalls den Einfluss der Zahnbürstenabnutzung auf die Plaquekontrolle. Bei über 70 Testpersonen, die über einen Zeitraum von 24 Stunden jegliche 
Mundhygienemaßnahmen eingestellt hatten, wurde der „Refined Modified Navy“Plaqueindex erhoben. Anschließend verwendete die Hälfte der Probanden eine neue Zahnbürste, während die andere Hälfte mit einer künstlich abgenutzten Zahnbürste, durch die eine Gebrauchsperiode von drei Monaten simuliert werden sollte, putzte. Nach einminütigem Zähneputzen wurde der Plaqueindex erneut erhoben und die Plaquereduktion der beiden Gruppen verglichen. Es stellte sich heraus, dass neue, nicht abgenutzte Zahnbürsten etwa ein Drittel mehr Plaque beseitigten als die künstlich abgenutzten Bürsten (DOHERTY et al. 1998).

Verschiedene Studien über die klinische Effizienz „natürlich abgenutzter“ Zahnbürsten zeigten hinsichtlich der Plaqueindizes nach dem Putzen keine statistisch signifikanten Unterschiede im Vergleich zu ungebrauchten, nicht abgenutzten Bürsten (CONFORTI et al. 2003, HEDGE et al. 2005, SFORZA et al. 2000, TAN und DALY 2002). DALY et al. untersuchten 1996 im Rahmen einer In-vivo-Studie den Abnutzungsstatus der verwendeten Zahnbürsten, indem die Vergrößerung der Bürstenoberfläche ermittelt und mit der zu Beginn der Studie gemessenen Oberfläche verglichen wurde. Trotz generell zunehmender, individuell jedoch deutlich variierender Abnutzungserscheinungen konnte anhand der ermittelten Plaquemenge bei den teilnehmenden Probanden keine verminderte Reinigungsfähigkeit festgestellt werden. Die Plaquekontrolle der Studienteilnehmer, die mit geringerem Kraftaufwand putzten, war folglich ebenso effektiv wie diejenige der Probanden, die eine gesteigerte Putzkraft aufwendeten. DEAN dagegen misst dem unterschiedlichen Kraftaufwand eine große Bedeutung für eine adäquate Plaqueentfernung bei. Er stellte 1991 in einer In-vitro-Studie mit Bürsten unterschiedlicher Abnutzungsgrade heraus, dass hinsichtlich der Entfernung künstlicher Plaque am Modell keine Korrelation zwischen der sichtbaren Bürstenabnutzung und der Reinigungseffizienz besteht. Er schloss daraus, dass individuelle Faktoren wie beispielsweise die manuelle Geschicklichkeit des Benutzers, die Putztechnik und die während des Putzens aufgewendete Kraft einen bedeutenderen Einfluss auf die Plaquekontrolle haben könnten als der Grad der Bürstenabnutzung (DEAN 1991).

Dass die Effektivität der Plaqueentfernung mit zunehmender Bürstenabnutzung abnimmt, konnten GLAZE und WADE 1986 in ihrer klinischen Studie konstatieren. Hier wurden 40 Studenten auf zwei Probandengruppen verteilt. Während die eine Gruppe über einen Zeitraum von zehn Wochen dieselbe Bürste anwendete, erhielten die Probanden der anderen Gruppe während der gleichen Zeitspanne alle zwei Wochen eine neue Zahnbürste. Vor Beginn der Studie erhielten alle Teilnehmer eine professionelle Zahnreinigung. Die sich anschließenden, 14-tägig stattfindenden Evaluationen umfassten nicht nur die Erhebung eines Plaque- und eines Gingivaindex, sondern auch die Bestimmung der jeweiligen Zahnbürstenabnutzung: 
Zum einen wurde der Abnutzungsgrad je nach Borstenzustand subjektiv eingeschätzt, und es erfolgte eine Klassifizierung der Zahnbürsten in „gut“, „mittelmäßig“ und „schlecht“, zum anderen wurde die aktiv bürstende Borstenoberfläche (in $\mathrm{mm}^{2}$ ) mit Hilfe eines speziellen Messzirkels ermittelt. Zwar stiegen die Werte des Plaqueindex zwischen Woche zwei und Woche zehn in beiden Gruppen an, jedoch zeigte sich, dass Probanden, die während der gesamten Versuchsperiode mit derselben Zahnbürste putzten, signifikant mehr Plaque aufwiesen als Probanden, die regelmäßig mit neuen Bürsten versorgt wurden. Die Reinigungseffektivität der getesteten Zahnbürsten sank mit wachsender Borstenermüdung. Hinsichtlich des Gingivaindex konnten keine statistisch signifikanten Unterschiede zwischen den beiden Gruppen festgestellt werden. Die subjektive Klassifizierung des Bürstenzustandes in „gut“, „mittelmäßig“ und „schlecht“ ergab, dass die Zahnbürsten einiger Probanden sich bereits nach zwei Wochen in einem schlechten Zustand befanden, während der Zustand anderer Zahnbürsten auch nach zehn Wochen noch als gut eingestuft wurde. Bei der Auswertung der mittels Zirkel gemessenen durchschnittlichen Größe des Borstenfeldes in $\mathrm{mm}^{2}$ wurde eine höhere Größenzunahme bei den länger benutzten Zahnbürsten festgestellt.

HEDGE et al. verglichen im Jahr 2005 mit Hilfe des „wear index“ (RAWLS et al. 1989) den Abnutzungstatus von Zahnbürsten, die nach drei Monaten ausgetauscht wurden (Gruppe II), mit demjenigen von Zahnbürsten nach einmonatigem Gebrauch (Gruppe I). Dieser Index wurde zur wissenschaftlichen Einstufung der Verschleißspuren einer Zahnbürste entwickelt und lässt die objektive Beurteilung eines aufgebogenen Borstenfeldes zu. Mittels des „wear index“ ist eine Kategorisierung beim Vergleich verschiedener Bürsten mit unterschiedlichem Ausmaß der „Borstenaufspreizung“ möglich. Nach HEDGE et al. variierte der Index zwischen 25 und 62\% in Gruppe II, in Gruppe I lagen die erhobenen Werte zwischen sechs bis 24\%, während sich beim Parameter Plaquebefall in beiden Gruppen keine signifikanten Unterschiede ergaben.

\subsubsection{Mikrobielle Besiedlung der Borsten}

Die im Rahmen der mechanischen Plaquekontrolle aus der Mundhöhle beseitigten pathogenen Mikroorganismen lagern sich der Zahnbürstenoberfläche an und können sich dort vermehren (NOGA et al. 1976, GLASS und LARE 1986, NIES et al. 2008). Das Ausmaß der Keimbesiedlung ist zumindest teilweise von der Gebrauchsdauer und dem Abnutzungsstatus einer Zahnbürste abhängig. Wann ein Zahnbürstenwechsel aus mikrobiologischer Sicht erfolgen sollte, ist nach derzeitigem wissenschaftlichen Kenntnisstand jedoch umstritten. Nicht selten, allerdings vor allem in der Laienpresse, wird empfohlen, die Zahnbürste nach Ausheilen einer 
Grippe bzw. eines grippalen Infektes auszutauschen, da andernfalls die Gefahr einer Reinfektion bestehe. In der wissenschaftlichen Literatur findet sich jedoch nur eine Studie, die eine Korrelation des Ausmaßes der bakteriellen Kontamination und der Gebrauchsdauer einer Zahnbürste belegt (HINGST 1989). Nach Ansicht des Autors sollte eine Zahnbürste nach drei, spätestens jedoch sechs Monaten ausgewechselt werden, außerdem immer dann, wenn entzündliche Erkrankungen des Mund- und Hals- bzw. Rachenraumes abgeklungen sind. Dass sich nur so eine Wiederansteckung durch die Verwendung der Zahnbürste verhindern lässt, äußert auch SPLIETH (2000). Aktuelle wissenschaftliche Untersuchungen setzen sich vermehrt mit dem potentiellen Reinfektionsrisiko durch die Keimbesiedlung täglich verwendeter Zahnbürsten auseinander. (BUNETEL et al. 2000, BÖSSMANN 2001, WARREN DP et al. 2001). Zwar weisen verschiedene andere Studien ebenfalls die bakterielle Kontamination von Zahnbürsten nach, jedoch verringert der Einsatz von Zahnpasten mit antibakteriellen Wirkstoffen die Überlebensrate und Menge der Mikroorganismen so deutlich, dass von einem sehr geringen Infektions- bzw. Reinfektionsrisiko ausgegangen werden kann (MALMBERG et al. 1994, TAJI und ROGERS 1998, BÖSSMANN 2001, QUIRYNEN et al. 2001, EFSTRATIOU et al. 2007).

In einer klinischen Studie untersuchten WETZEL et al. die mikrobielle Zusammensetzung der Flora auf gebrauchten Zahnbürsten. Auf den verschiedenen Zahnbürsten, welche von Kindern zwischen sechs und 13 Jahren benutzt worden waren, fand man Mutansstreptokokken am häufigsten, gefolgt von Laktobazillen; seltener zeigte sich Candida albicans (WETZEL et al. 2005).

\subsubsection{Verletzungsgefahr durch abgenutzte Borsten}

Abgenutzte Borsten können verbogen, abradiert oder aufgezwirbelt sein und so möglicherweise zu Verletzungen der oralen Hart- und Weichgewebe führen. Über Traumatisierungen, die durch Zahnbürsten provoziert werden, wird in der Literatur vielfach berichtet. Neben gingivalen Rezessionen (SERINO et al. 1994) und Zahnhalsdefekten (BERGSTRÖM und LAVSTEDT 1979) werden auch gingivale Erosionen beschrieben (SANDHOLM et al. 1982). Ein Indiz auf eine potentiell erhöhte Verletzungsgefahr durch abgenutzte Borsten findet sich in der wissenschaftlichen Literatur allerdings bis heute nicht.

GLAZE und WADE (1986) konnten bei der gingivalen Beurteilung keine statistisch signifikanten Unterschiede zwischen Probanden, die über zehn Wochen mit derselben Zahnbürste putzten, und Probanden, die alle zwei Wochen eine neue Zahnbürste verwendeten, feststellen. Andere Autoren gehen davon aus, dass Zahnbürsten erst nach zweimonatigem Gebrauch den 
Anforderungen an eine zufriedenstellende Endabrundung gerecht werden, während dies zu Studienbeginn nicht bei allen Bürsten der Fall war (BIENENGRÄBER et al. 1995). KREIFELDT et al. beschrieben 1980, dass bereits benutzte Borsten tendenziell weicher und daher hinsichtlich der Plaquereduktion ineffektiver werden. Von einem durch Zähneputzen hervorgerufenen Trauma ist in keiner der erwähnten Studie die Rede.

\subsubsection{Abnutzung, Reinigungswirkung und Bürstenkopfwechsel bei elektrischen}

\section{Zahnbürsten}

Über die Abnutzung und das Austauschintervall elektrischer Zahnbürsten finden sich in der Literatur bislang nur wenige Studien. Untersuchungen von Handzahnbürsten sind nicht direkt auf elektrische Zahnbürsten zu projizieren. Nach Herstellerangaben sollte jedoch, ähnlich wie bei den Handzahnbürsten, ein regelmäßiger Austausch nach drei Monaten stattfinden. Wissenschaftliche Grundlagen für diese Empfehlung existieren allerdings kaum.

CONFORTI et al. stellten beim Vergleich neuer und gebrauchter Bürstenköpfe keine statistisch signifikanten Unterschiede hinsichtlich der Plaquereduktion fest. Einzig die Auswertung des Parameters Plaquebefall derjenigen Untergruppe, deren Bürsten als „schwer“ bzw. „extrem abgenutzt“ eingeschätzt wurden, ergab signifikant schlechtere Werte im Vergleich zu neuen Zahnbürsten (CONFORTI et al. 2003). Eine weitere Untersuchung stellte heraus, dass Bürstenköpfe eines rotierend-oszillierenden Gerätes nach dreimonatiger Anwendung ebenso gründlich reinigten wie neue Bürstenköpfe. Traumatisierungen der Gingiva wurden weder bei Probanden, die mit neuen, noch bei Probanden, die mit abgenutzten Bürstenköpfen putzten, festgestellt (HOGAN et al. 2007).

Wie bei den bereits erwähnten Studien mit manuellen Zahnbürsten, treten auch beim Abnutzungsstatus elektrischer Zahnbürsten nach dreimonatigem Gebrauch deutliche Unterschiede auf. Einige Autoren sind daher der Auffassung, dass die Abnutzung der Borsten allein keinen Bürstenwechsel rechtfertige und somit ein Austauschintervall von drei Monaten nicht unterstützt werden könne (HOGAN et al. 2007). 


\section{Material und Methoden}

\subsection{Administrative Vorbereitungen}

Die generelle Erlaubnis zur Durchführung der Studie wurde bei der Ethik-Kommission der Georg-August-Universität Göttingen beantragt und durch deren Vorsitzenden unter der Antragsnummer 19/03/2007 genehmigt.

\subsection{Probanden}

Die Durchführung der vorliegenden Studie erforderte die freiwillige Teilnahme von Testpersonen über 18 Jahren. Probanden, auf die einer der folgenden Punkte zutraf, wurden von der Studienteilnahme ausgeschlossen:

- Immunsuppression

- Organtransplantation

- Notwendigkeit einer Endokarditisprophylaxe

- Vorliegen von Hepatitis A, B, C, TBC, HIV

- Niereninsuffizienz

- Anfalls- oder Nervenleiden

- Suchterkrankungen

- Schwangerschaft

- Probanden mit weniger als 20 Zähnen

- Probanden mit festsitzenden kieferorthopädischen Apparaturen.

\subsection{Testzahnbürsten und Testzahnpasta}

In der Studie wurden drei verschiedene elektrische Zahnbürsten hinsichtlich des Einflusses ihrer Gebrauchsdauer auf Plaquekontrolle und Ginigivazustand untersucht.

\subsubsection{Rotierend-oszillierende Zahnbürste}

Testzahnbürste 1 war die rotierend-oszillierende ProfessionalCare ${ }^{\mathrm{TM}} 7000$ (Procter\&Gamble Service GmbH, Schwalbach am Taunus, Kronberg, Deutschland). Der in der Studie untersuchte FlexiSoft@-Bürstenkopf verfügt über ein rundes, konkaves Borstenfeld mit 26 kreisförmig angeordneten Borstenbüscheln von je 60 Filamenten.

Die insgesamt sechs blau gefärbten, außen angeordneten Borstenbüschel zeigen an, wann ein Auswechseln der Aufsteckbürste indiziert ist, indem ihre Farbe mit steigender Gebrauchs- 
dauer allmählich verblasst. Wenn die Indicator ${ }^{\circledR}$ Borsten bis zur Hälfte ihrer Länge entfärbt sind, im Mittel nach etwa drei Monaten, sollte der Bürstenkopf nach Angaben des Herstellers ausgetauscht werden.

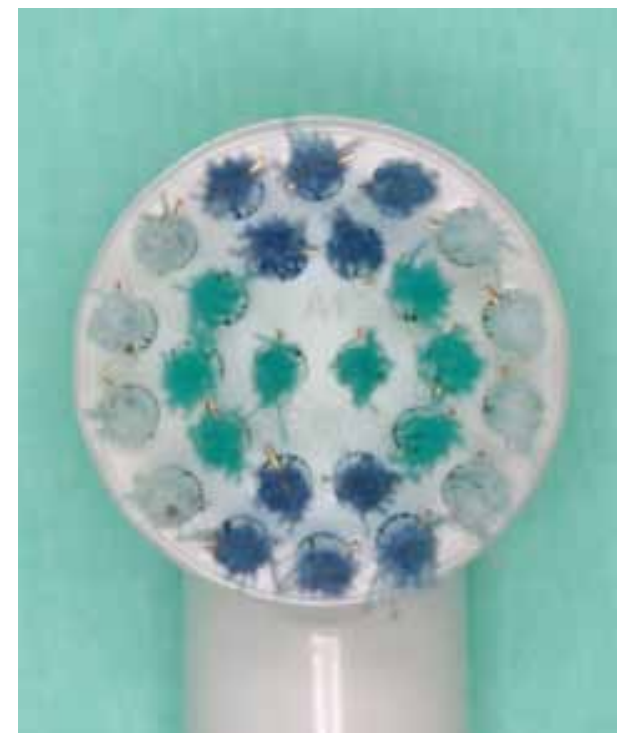

Abb. 1: FlexiSoft@-Bürste (Procter\&Gamble), Borstenfeld

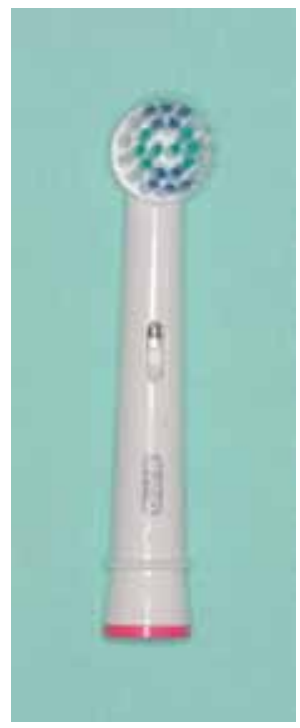

Abb. 2: Draufsicht

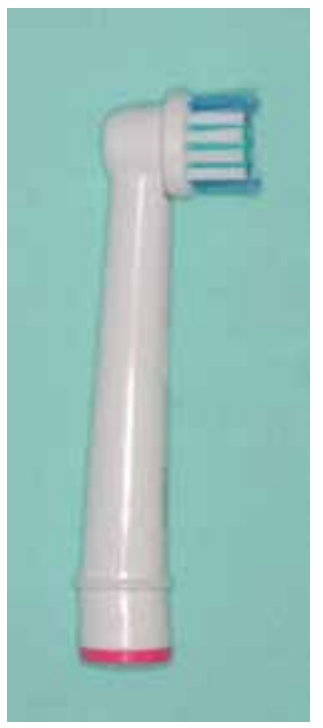

Abb. 3: Seitenansicht

Die verbesserte Reinigungswirkung des Gerätes soll auf der 3D-Technologie beruhen: Neben 40.000 Rotationsbewegungen pro Minute führt der Bürstenkopf sowohl Pulsations-, als auch Oszillationsbewegungen aus. Eine integrierte Andruckkontrolle stoppt pulsierende Bewegungen zum Schutz der Ginigiva bei einem aufgewendeten Zahnputzdruck von > 2,5 Newton, rotierend-oszillierende Bewegungen hingegen werden fortgeführt. Ein zum Gerät gehöriger Timer signalisiert dem Benutzer nach jeweils 30 Sekunden einen Wechsel zum nächsten Quadranten. Außerdem kann zwischen zwei Geschwindigkeitsstufen gewählt werden, wobei Stufe 1 für die Massage der Gingiva und Stufe 2 für die Zahnreinigung einzustellen ist. Im Lieferumfang der Zahnbürste ist ein Akkuladegerät mit einem Netzteil von 240 Volt/ 60 Hertz enthalten.

\subsubsection{Schallaktive Zahnbürste}

Die schallaktive SoniCare ${ }^{\mathrm{TM}}$ - Zahnbürste (Philips GmbH, Hamburg, Deutschland) diente als Testzahnbürste 2. Dieses Gerät verfügt über einen länglichen Bürstenkopf und arbeitet mit einer Frequenz von 260 Hertz. Dies entspricht 31.000 Bürstbewegungen pro Minute. Das Borstenfeld der in dieser Studie verwendeten ProResults Standard-Aufsteckbürste ist multi- 
tufted und weist 32 ellipsoid angeordnete Borstenbüschel mit je 50 Borsten unterschiedlicher Längen und Farben auf.

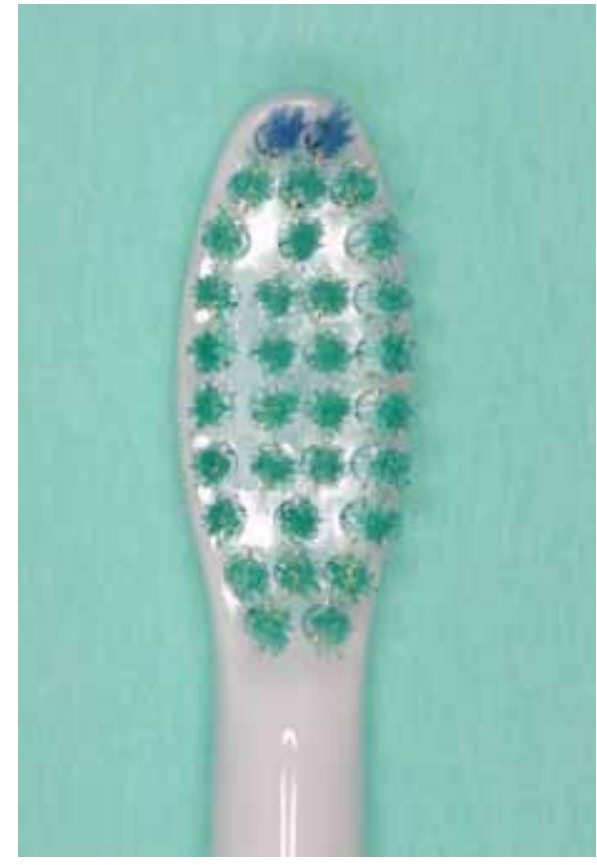

Abb. 4: ProResults-Bürste (Philips), Borstenfeld

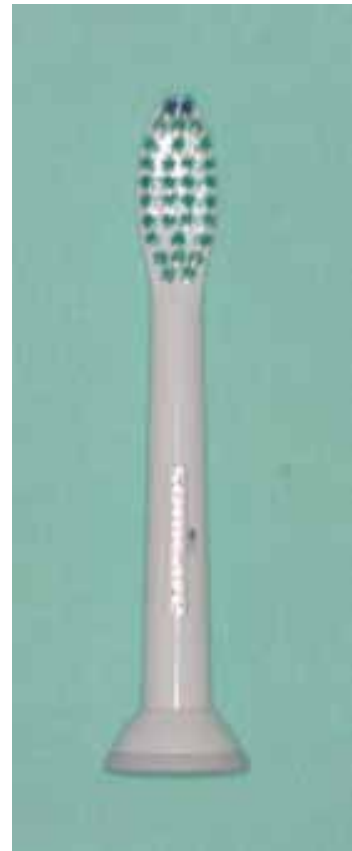

Abb. 5: Draufsicht

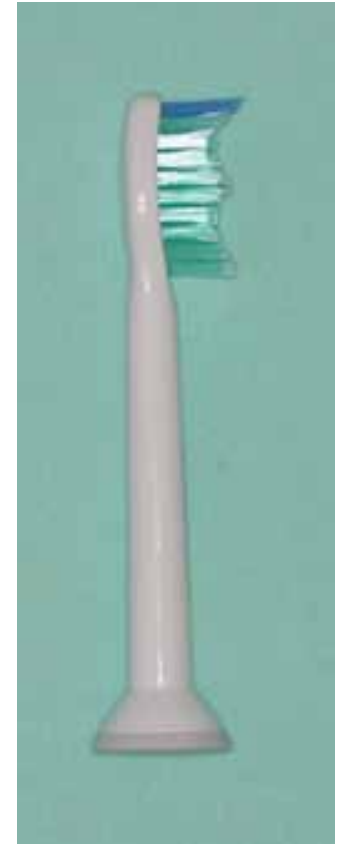

Abb. 6: Seitenansicht

Die Zahnbürste ist mit einem quadpacer ${ }^{\circledR}$ ausgestattet, der dem Benutzer nach 30, 60 und 90 Sekunden die Notwendigkeit eines Wechsels zum nächsten Quadranten signalisiert. Bei dieser Zahnbürste ist ab Werk eine easy start ${ }^{\mathrm{TM}}$ - Funktion eingestellt, welche laut Hersteller eine einfachere Gewöhnung des Nutzers an schallbetriebene Zahnbürsten ermöglichen soll: Die optimale Leistung der Zahnbürste wird erst nach zwölfmaliger, jeweils einminütiger Benutzung durch allmähliche Steigerung erreicht. Für die Durchführung der vorliegenden klinischen Studie wurde diese Funktion deaktiviert. Des Weiteren verfügt das Gerät über drei unterschiedliche Putzmodi; in der vorliegenden Untersuchung wurde der standardmäßig eingestellte zweiminütige Clean-Modus ausgewählt. Zum Lieferumfang des Gerätes gehören die Akkuladestation mit einem Netzteil von 240 Volt/60 Hertz, ein Reise-Akkuladegerät und ein UV-Desinfektionsgerät.

\subsubsection{Elektrisch unterstützte Handzahnbürste}

Testzahnbürste 3 war die Dr. Best Vibration ${ }^{\circledR}$ mittel (GlaxoSmithKline, München, Deutschland) eine elektrisch unterstützte Handzahnbürste. Diese Zahnbürste ist batteriebetrieben und kann, laut Herstellerangaben, bei zweimaligem, jeweils zweiminütigem Putzen pro Tag über einen Zeitraum von etwa drei Monaten benutzt werden. Nach Ablauf der drei Monate ist die 
Batterie leer; ein Aufladen beziehungsweise Austauschen der Batterie ist nicht möglich. Die Zahnbürste weist ein Borstenfeld mit 51 Büscheln von jeweils 42 Filamenten unterschiedlicher Längen und Farben auf.

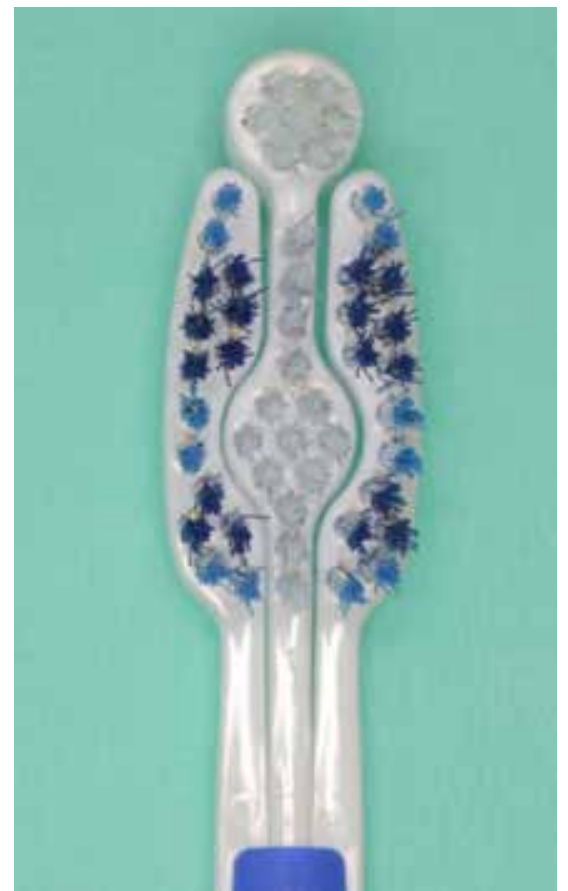

$\begin{array}{ll}\text { Abb. 7: } & \begin{array}{l}\text { Dr. Best Vibration }{ }^{\circledR} \\ \text { (GlaxoSmithKline), }\end{array}\end{array}$

$\begin{array}{ll}\text { Abb. 7: } & \begin{array}{l}\text { Dr. Best Vibration } \\ \end{array} \\ & \text { (GlaxoSmithKline), }\end{array}$ Borstenfeld

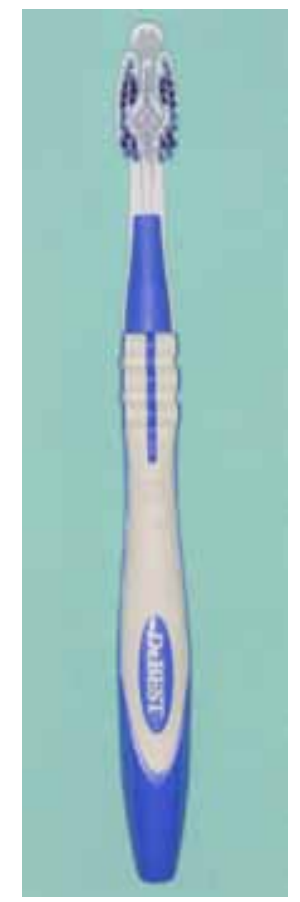

Abb. 8: Draufsicht

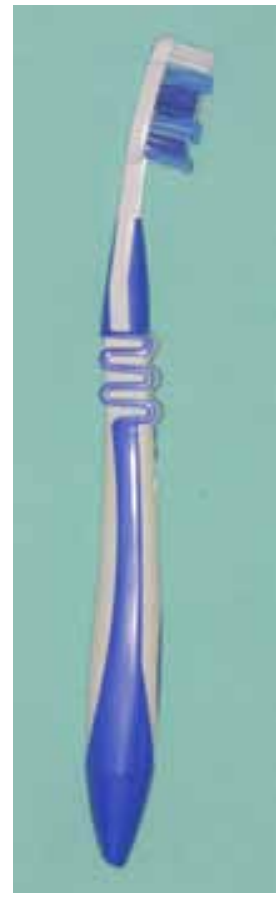

Abb. 9: Seitenansicht

Abb. 8. Draufsicht

Abb. 9: Seitenansicht 


\subsection{Mundhygiene-Indizes und Plaquerelevator}

\subsubsection{Indizes}

\subsubsection{Plaqueindex}

Die Beurteilung der Plaqueausdehnung erfolgte durch den nach Turesky (TURESKY et al. 1972) modifizierten Quigley-Hein-Index (QUIGLEY und HEIN 1962). Der Index bewertet den Plaquebefall der koronalen Zahnoberflächen. Vor der Erhebung werden die Zahnoberflächen mit einem Plaque-Revelator eingefärbt. Folgende sechs Schweregrade werden unterschieden:

Grad 0: keine Plaque

Grad 1: vereinzelte Plaqueinsel

Grad 2: deutlich zusammenhängende, bis zu $1 \mathrm{~mm}$ breite Plaquelinie am

Gingivalrand

Grad 3: Plaqueausdehnung im zervikalen Drittel

Grad 4: Plaqueausdehnung in das mittlere Drittel

Grad 5: Plaqueausdehnung bis in das koronale Drittel

Die Oberfläche jedes zu bewertenden Zahnes wird in jeweils drei vestibuläre und drei orale Areale unterteilt, wodurch sich sechs Messpunkte pro Zahn ergeben.

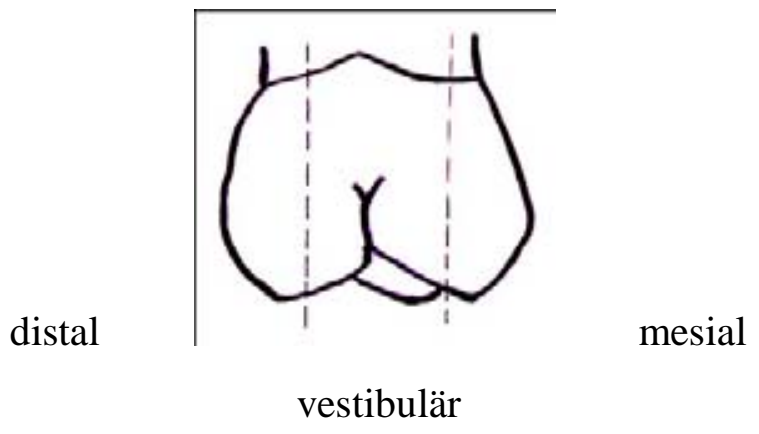

Abb. 10: QHI, Flächeneinteilung des zu bewertenden Zahnes (STEINLE 2003, S.32)

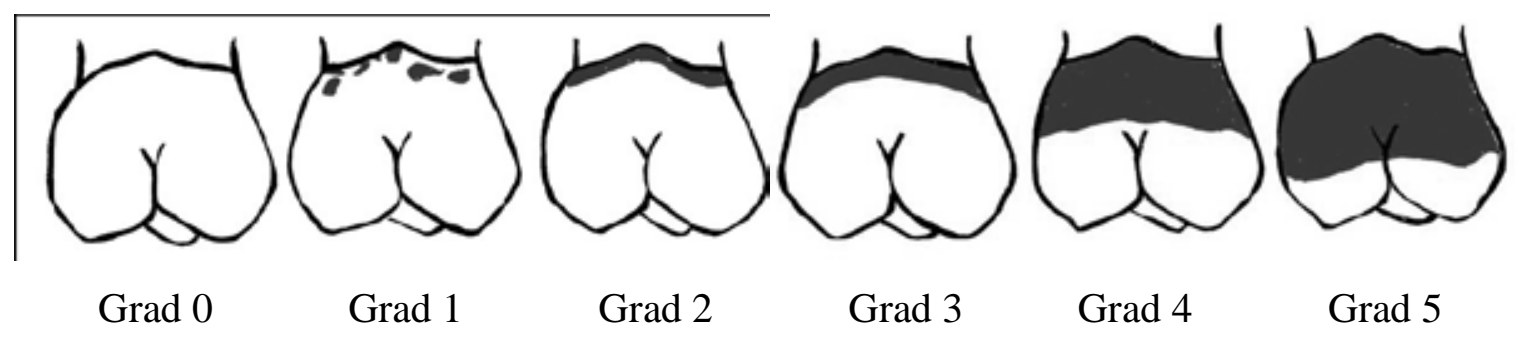

Abb. 11: Graduierung des Plaquebefalls beim QHI

(STEINLE 2003, S.32) 
Der Plaquebefall von überkronten Zähnen und Weisheitszähnen wird nicht mitbewertet.

Der QHI wird als Mittelwert aller Einzelwerte angegeben.

\subsubsection{Gingivaindizes}

Papillen-Blutungs-Index (SAXER und MÜHLEMANN 1975)

Für die Erhebung des PBI wird eine Parodontalsonde von der Basis bis zur Papillenspitze im Bereich des Sulkuseingangs entlang geführt. Der PBI wird in allen vier Quadranten erhoben, wobei alternierend fazial und oral sondiert werden kann. Der PBI kann als Blutungszahl (Summe aller Einzelwerte) oder als Index (Mittelwert aller Einzelwerte) ausgedrückt werden. Unterschieden werden fünf Grade, in denen die Blutungsintensität beurteilt wird (MÜLLER 2001):

Grad 0: keine Blutung

Grad 1: Auftreten eines Blutungspunktes

Grad 2: Auftreten mehrerer Blutungspunkte oder eines schmalen Blutbandes

Grad 3: Ausfüllen des interdentalen Dreiecks mit Blutung unmittelbar nach Sondierung

Grad 4: profuse Blutung nach Sondierung, die Blutung dehnt sich über die marginale Gingiva aus, evtl. mit Tropfenbildung

\section{Gingivaindex nach LÖE und SILNESS (1963)}

Bei diesem Index wird die dem zu bewertenden Zahn anliegende Gingiva in vier Abschnitte unterteilt, von denen drei auf der vestibulären Seite liegen. Es werden vier Bewertungsgrade unterschieden (MÜLLER 2001):

Grad 0: normale Gingiva

Grad 1: leichte Entzündung mit leichten Farbveränderungen, leichtes Ödem, keine Blutung bei Palpation

Grad 2: mäßige Entzündung mit Rötung, Ödem und Blutung bei Ausstreichen mit der Sonde

Grad 3: schwere Entzündung, ausgeprägte Rötung und Ödembildung, Ulzerationen, Tendenz zur Spontanblutung

Der GI-Wert errechnet sich durch Bildung des Mittelwertes der Einzelwerte jedes Zahnes. Für die Erhebung wird eine Parodontalsonde horizontal im Bereich des Sulkuseingangs am Gingivalrand entlang geführt. Die GI-Werte können mit verschiedenen Ausprägungsgraden der Gingivitis in Verbindung gebracht werden: 
Tabelle 1: Gingivaindex und Ausprägungsgrad der gingivalen Entzündung

\begin{tabular}{|cc|}
\hline GI-Werte & Grad der Gingivitis \\
$0,1-1$ & Mild \\
$1,1-2$ & Mäßig \\
$2,1-3$ & Schwer \\
\hline
\end{tabular}

Die Parodontien der dritten Molaren werden nicht evaluiert.

\subsubsection{Plaquerevelator}

Um die auf den Zahnoberflächen befindlichen Beläge sichtbar zu machen, wurden diese vor Erheben des Plaqueindex mit Erythrosin-Lösung rot angefärbt.

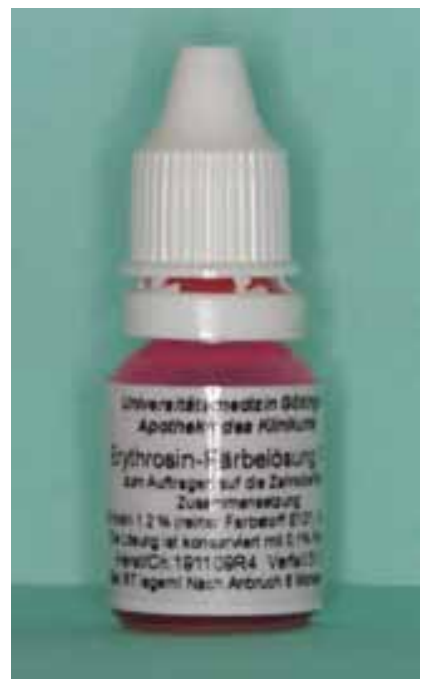

Abb. 12: Erythrosin-Lösung

\subsection{Einteilung der Gruppen}

- Gruppe A: Testzahnbürste 1, Verwendung über 24 Wochen

- Gruppe B: Testzahnbürste 1, Verwendung über 24 Wochen, hierbei alle vier Wochen ein neuer Bürstenkopf

- Gruppe C: Testzahnbürste 2, Verwendung über 24 Wochen

- Gruppe D: Testzahnbürste 2, Verwendung über 24 Wochen, hierbei alle vier Wochen ein neuer Bürstenkopf

- Gruppe E: Testzahnbürste 3, Verwendung bis Ende der Batterie-Laufzeit

- Gruppe F: Testzahnbürste 3, Verwendung über 16 Wochen, hierbei alle vier Wochen eine neue Zahnbürste 
Nach Ablauf der 24 bzw.16 Wochen erhielten die Probanden aller Gruppen einen neuen Bürstenkopf bzw. eine neue Zahnbürste. Die Abschlussuntersuchung erfolgte sieben bis zehn Tage nach dem letzten Kontrolltermin (Abb. 13).

\subsection{Studiendesign}

Die Untersuchung wurde im einfach-blinden Paralleldesign durchgeführt, das heißt, jeder Proband putzte mit nur einer Zahnbürste, und der Untersucher wusste nicht, über welchen Zeitraum der jeweilige Proband die zugewiesene Zahnbürste benutzte.

\subsection{Studienablauf}

\subsubsection{Anamnese und Einverständniserklärung}

Die Teilnahme der Probanden an der Studie war freiwillig, eine Nichtteilnahme zog keinerlei Konsequenzen nach sich. Mit Hilfe eines Anamnesebogens sollten die oben genannten Ausschlusskriterien erfasst und Zahnputzgewohnheiten erfragt werden. Die Probanden wurden über die Studie aufgeklärt und gebeten, eine Einverständniserklärung zu unterschreiben.

\subsubsection{Klinisch-zahnmedizinische Erstuntersuchung}

Bei der klinischen Untersuchung wurde eine intraorale Inspektion vorgenommen, um evtl. vorhandene Auffälligkeiten bzw. Reizfaktoren zu erfassen, sowie ein dentaler Befund mit Hilfe des DMF-T-Index erhoben. Der DMF-T-Index beurteilt die Anzahl der kariösen (D decayed), fehlenden (M missing) und gefüllten (F filled) Zähne (T teeth) (KLEIN et al. 1938).

\subsubsection{Erheben der Indizes}

Die Mundhygiene der Probanden wurde durch die Erhebung eines Plaqueindex (QHI) und zweier Gingivaindizes (PBI, GI) eingestuft.

\subsection{Randomisierung und Instruktion der Probanden/Zahnreinigung}

Die Verteilung der Studienteilnehmer auf die Versuchsgruppen erfolgte anhand des in der Screening-Untersuchung gemessenen Plaqueindex. Die Probanden wurden gemäß QHI in drei Mundhygiene-Kategorien (gut, mittelmäßig, schlecht) eingestuft und anschließend per Losverfahren gleichmäßig auf die sechs Gruppen A-F verteilt. Zudem erfolgte eine Randomisierung der Testpersonen nach Rechts- bzw. Linkshändigkeit. 
Nach der klinischen Untersuchung und der Erhebung der Indizes wurde bei allen Probanden zu Beginn der Studie (Tag 1) eine professionelle Zahnreinigung (PZR) durchgeführt. Vorhandene Reizfaktoren und nicht natürliche Plaqueretentionsstellen wurden vor Studienbeginn beseitigt. Die Probanden erhielten eine Mundhygieneunterweisung sowie eine Instruktion in den Umgang mit der von ihnen zu benutzenden Zahnbürste. Es folgten eine Aufklärung über den Studienablauf und die Ausgabe von Zahnbürste und Zahnpasta. Die Probanden wurden aufgefordert, zweimal täglich für jeweils zwei Minuten sowie unmittelbar vor jeder Kontrolluntersuchung mit der ihnen zugewiesenen Zahnbürste zu putzen. Außerdem wurden sie angewiesen, über den gesamten Zeitraum der Studie auf den Gebrauch von Hilfsmitteln zur Interdentalraumpflege zu verzichten. Probanden der Gruppen A-D wurden darauf hingewiesen, dass der Akku der ihnen ausgehändigten Zahnbürste während der gesamten Laufzeit der Studie stets vollständig geladen sein sollte. Probanden der Gruppen E wurden gebeten, dem Untersucher schnellstmöglich das Ende der Batterielaufzeit ihrer Zahnbürste zu melden, um einen Termin für die sich dadurch ergebende Kontrolluntersuchung zu vereinbaren und um eine durchschnittliche Gebrauchsdauer der batteriebetriebenen Geräte zu ermitteln.

\subsection{Untersuchungs- und Kontrollintervall}

Der erste Kontrolltermin fand zwei Wochen nach Studienbeginn (Tag $14 \pm 3$ ) statt. Weitere Kontrolltermine fanden acht Wochen ( \pm 3 Tage), 12 Wochen ( \pm 3 Tage), 16 Wochen ( \pm 3 Tage) und 24 Wochen ( \pm 3 Tage) nach Studienbeginn statt. In den Kontrollterminen wurden wie bei der Eingangsuntersuchung der Plaqueindex (QHI) und die Gingivaindizes (PBI und GI) erhoben. Nach Ablauf der 24 bzw. 16 Wochen erhielten alle Probanden eine neue Aufsteckbürste bzw. Zahnbürste.

\subsection{Abschlussuntersuchung}

Die Abschlussuntersuchung erfolgte sieben bis zehn Tage nach dem letzten Kontrolltermin. Hier wurden ebenfalls der Plaqueindex und die Gingivaindizes erhoben. 
Erstuntersuchung und Probandenscreening (Tag 0):

- Anamnese und Befund

- Erhebung von Plaqueindex (QHI) und Gingivaindizes (PBI und GI)

- Aufklärung und Einverständniserklärung

- Randomisierung: Plaqueindex (QHI) und Rechts-/Linkshänder

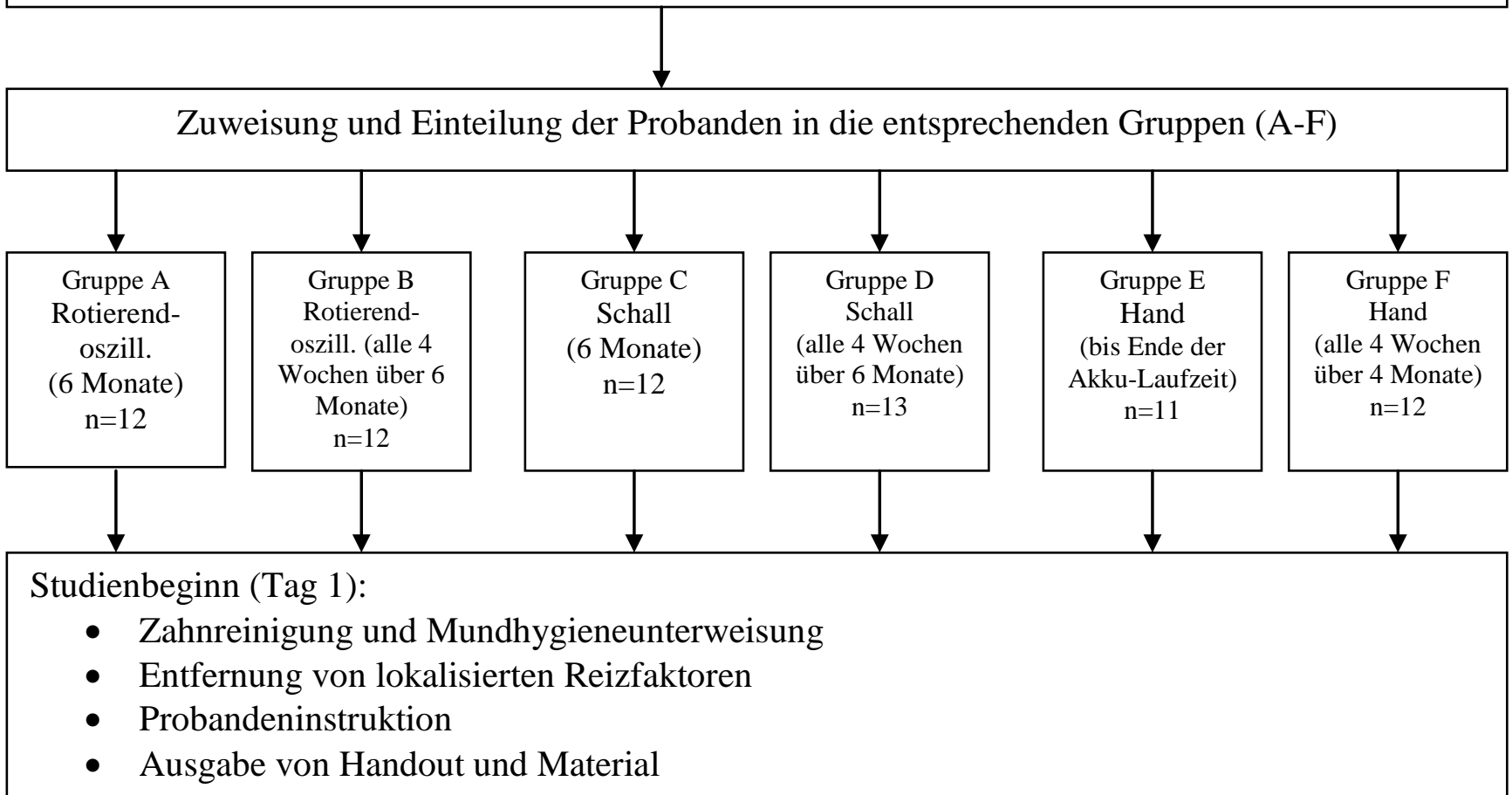

Kontrolle/Recall:

- Erhebung von Plaqueindex (QHI) und Gingivaindizes (PBI und GI)

- Patientenbefragung:

- 2 Wochen nach Studienbeginn

- 8 Wochen nach Studienbeginn

- 12 Wochen nach Studienbeginn

- 16 Wochen nach Studienbeginn

- 24 Wochen nach Studienbeginn (Gruppe A-D)

$\rightarrow$ Ausgabe neuen Materials

Abschlussuntersuchung (16 bzw. 24 Wochen $+7-10$ Tage):

- Erhebung von Plaqueindex (QHI) und Gingivaindizes (PBI und GI)

Abb. 13: Graphische Darstellung des Versuchsablaufs im Flussdiagramm 


\subsection{Dokumentation}

Die Erfassung der erhobenen Befunde und Indizes erfolgte gemäß Prüfplan mittels Prüfbögen.

Die Probanden wurden gemäß Erfassungsbogen am Tag 0 untersucht, anschließend wurde ihnen eine laufende Nummer (1XX) zugeordnet.

\subsection{Auswertung der Ergebnisse}

Die Auswertung der Ergebnisse erfolgte zusammen mit der Abteilung Medizinische Statistik (Direktor: Prof. Dr. Tim Friede) der Universitätsmedizin Göttingen (Arbeitsgruppe „Klinische Studien“, Leiter: Prof. Dr. Reinhard Hilgers, Prof. Dr. Tim Friede).

\subsubsection{Gruppen A-D}

Für die statistische Auswertung der Gruppen A-D wurden, wie 2003 von BARTHKE und BRUNNER beschrieben, Rangverfahren für faktorielle Versuchsanlagen mit einer Adjustierung im Sinne einer nichtparametrischen Kovarianzanalyse mit den Faktoren Zahnbürstentyp, Zeitpunkt und Verschleißgruppe sowie der Kovariablen Baseline-Wert durchgeführt. Zur Analyse wurde die SAS-Prozedur (SAS 9.2) PROC MIXED verwendet. Die Adjustierung des multiplen Niveaus bei den Vergleichen gegen den Baselinewert erfolgte nach DUNNETT (1955).

\subsubsection{Gruppen E und F}

Da aufgrund der limitierten Batterielaufzeit der elektrisch unterstützten Handzahnbürsten eine Studiendauer von sechs Monaten nicht möglich war und somit ein unmittelbarer Vergleich mit den beiden anderen Testzahnbürsten wenig sinnvoll erscheint, wurde auf eine statistische Auswertung, wie sie in den Gruppen A-D erfolgte, verzichtet. Daher wurden die Ergebnisse der in den Gruppen E und F untersuchten Zahnbürsten deskriptiv aufgeführt (Vgl. Kapitel 4.6). 


\section{Ergebnisse}

\subsection{Patientenkollektiv (Gruppen A-F)}

An der Studie nahmen 71 Probanden im Alter zwischen 20 und 70 Jahren teil, darunter 43 Frauen und 28 Männer. Das durchschnittliche Alter der Teilnehmer betrug 28.9 \pm 10.7 Jahre (Tabelle 2). Unter ihnen befanden sich 17 Raucher und 55 Nichtraucher. Neunzehn Probanden putzten vor der Studienteilnahme gewohnheitsgemäß ausschließlich mit manuellen Zahnbürsten, 42 Teilnehmer benutzten vorher elektrische Zahnbürsten, elf Probanden verwendeten zuvor sowohl manuelle als auch elektrische Zahnbürsten.

Tabelle 2: Probandencharakteristik für alle Probanden nach Gruppen

\begin{tabular}{|c|c|c|c|c|c|c|c|}
\hline & Insgesamt & A & B & C & D & $E$ & $\mathrm{~F}$ \\
\hline $\begin{array}{l}\text { Probanden- } \\
\text { anzahl (N) }\end{array}$ & $n=71$ & $n=12$ & $n=12$ & $n=12$ & $n=13$ & $n=11$ & $\mathrm{n}=12$ \\
\hline $\begin{array}{l}\text { Drop outs } \\
(\mathrm{N})\end{array}$ & $\mathrm{n}=1$ & $\mathrm{n}=0$ & $n=0$ & $\mathrm{n}=0$ & $n=0$ & $\mathrm{n}=1$ & $n=0$ \\
\hline $\begin{array}{c}\text { Alter } \\
(\mathrm{MW} \pm \mathrm{SD})\end{array}$ & $28.9 \pm 10.7$ & $24.1 \pm 2.5$ & $33.5 \pm 17.9$ & $26.5 \pm 2.5$ & $30.0 \pm 8.9$ & $26.1 \pm 5.2$ & $33.2 \pm 14.8$ \\
\hline $\begin{array}{c}\text { Geschlecht } \\
\hat{\delta}\end{array}$ & $n=28$ & $n=5$ & $n=5$ & $n=5$ & $n=3$ & $n=3$ & $n=7$ \\
\hline $\begin{array}{l}\text { Linkshänder } \\
\text { (N) }\end{array}$ & $n=0$ & $n=0$ & $n=0$ & $n=0$ & $n=0$ & $n=0$ & $n=0$ \\
\hline $\begin{array}{c}\text { DMF-T } \\
(\mathrm{MW} \pm \mathrm{SD})\end{array}$ & $8.1 \pm 5.4$ & $5.6 \pm 5.8$ & $8.8 \pm 4.7$ & $6.7 \pm 3.8$ & $10.5 \pm 6.0$ & $8.1 \pm 5.2$ & $8.8 \pm 6.0$ \\
\hline
\end{tabular}

\subsection{QHI (Gruppen A-D)}

Unabhängig vom verwendeten Zahnbürstentyp (rotierend-oszillierend/schallaktiv) ließ sich eine signifikante Wechselwirkung zwischen Zeitpunkt und Verschleißgruppe nachweisen ( $p=0.001$ ). Das heißt, der zeitliche Verlauf des QHI zeigt signifikante Unterschiede zwischen der Gruppe der 6-Monats-Anwender und der Gruppe der 4-Wochen-Anwender. Die Auswertung des Plaqueindex zeigte in allen Gruppen eine Verbesserung am ersten Kontrolltermin (nach 14 Tagen) im Vergleich zur Ersterhebung an Tag 0 (Tabelle 3). 
Tabelle 3: Gruppenabhängige Mittelwerte (MW) \pm Standardabweichung (SD) und Medianwerte für den Parameter QHI zu allen Untersuchungszeitpunkten

\begin{tabular}{|ccccc|}
\hline Zeitpunkt & $\mathrm{A}$ & $\mathrm{B}$ & $\mathrm{C}$ & $\mathrm{D}$ \\
& $0.91 \pm 0.54$ & $0.88 \pm 0.53$ & $0.89 \pm 0.61$ & $1.05 \pm 0.55$ \\
Baseline & $(0.92)$ & $(0.84)$ & $(0.80)$ & $(1.02)$ \\
& & & & \\
Tag 14 & $0.72 \pm 0.33$ & $0.59 \pm 0.30$ & $0.71 \pm 0.38$ & $0.84 \pm 0.40$ \\
& $(0.68)$ & $(0.60)$ & $(0.64)$ & $(0.83)$ \\
& $0.77 \pm 0.30$ & $0.65 \pm 0.33$ & $0.65 \pm 0.36$ & $0.86 \pm 0.41$ \\
Woche 8 & $(0.76)$ & $(0.70)$ & $(0.46)$ & $(0.73)$ \\
& $0.81 \pm 0.36$ & $0.76 \pm 0.60$ & $0.63 \pm 0.33$ & $0.88 \pm 0.34$ \\
Woche 12 & $(0.77)$ & $(0.70)$ & $(0.51)$ & $(0.76)$ \\
& & & & \\
Woche 16 & $0.85 \pm 0.41$ & $0.70 \pm 0.38$ & $0.74 \pm 0.35$ & $0.83 \pm 0.30$ \\
& $(0.81)$ & $(0.84)$ & $(0.69)$ & $(0.89)$ \\
& $1.08 \pm 0.38$ & $0.67 \pm 0.40$ & $0.93 \pm 0.43$ & $0.87 \pm 0.28$ \\
Woche 24 & $(1.08)$ & $(0.76)$ & $(0.91)$ & $(0.86)$ \\
& & & & \\
& $0.68 \pm 0.27$ & $0.62 \pm 0.36$ & $0.69 \pm 0.32$ & $0.77 \pm 0.26$ \\
Woche 25 & $(0.69)$ & $(0.57)$ & $(0.65)$ & $(0.73)$ \\
& & & &
\end{tabular}

\subsubsection{Oral-B Professional Care}

Im Zeitraum zwischen dem ersten (Tag 14) und dem vorletzten Kontrolltermin (Woche 24) stieg der Mittelwert des QHI bei den Probanden, die sechs Monate dieselbe Zahnbürste verwendeten, von $0.72 \pm 0.33$ auf $1.08 \pm 0.38$. Bei der Abschlussuntersuchung (Woche 25) hingegen wurden im Vergleich zu Woche 24 erheblich niedrigere Werte erhoben (0.68 \pm 0.27$)$. Die ermittelten Werte derjenigen Testpersonen, die alle vier Wochen mit einer neuen Zahnbürste putzten, blieben zwischen Tag 14 und Woche 24 auf einem ähnlichen Niveau; jedoch zeigte sich auch hier ein geringerer Mittelwert bei der Abschlussuntersuchung (Woche 25) im Vergleich zur Erhebung nach sechs Monaten (Abb. 14). 
Bürstentyp: rotierend

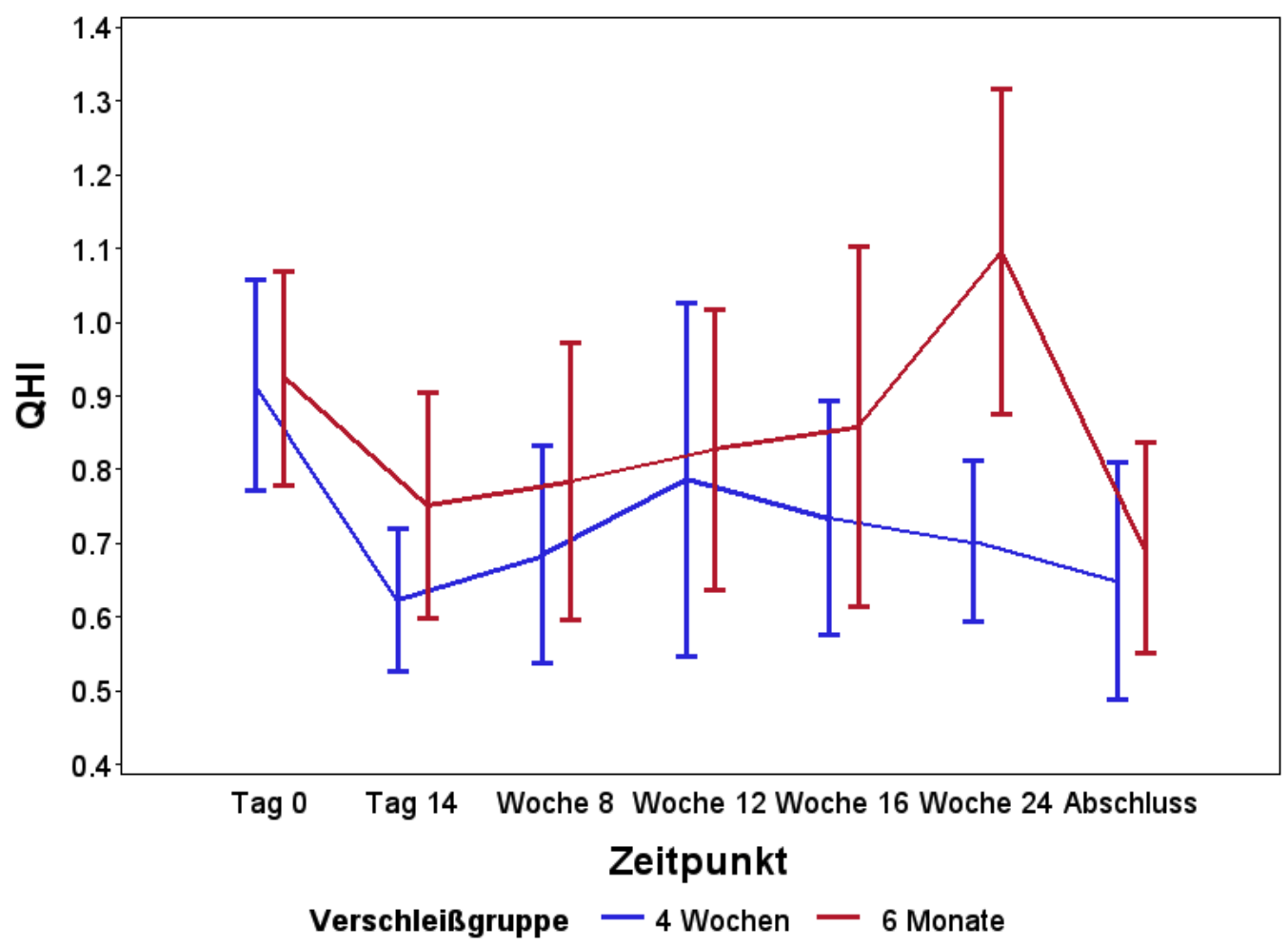

Abb. 14: Verlauf des QHI in den Gruppen A und B. Mittelwerte und 95\%-Konfidenzintervalle zu den jeweiligen Messzeitpunkten. Die zu den einzelnen Untersuchungszeitpunkten ermittelten Mittelwerte sind durch eine Linie verbunden.

\subsubsection{Philips SoniCare}

Bei den Testpersonen, die die Zahnbürste alle vier Wochen wechselten, blieb der QHI zwischen Tag 14 und Woche 24 in etwa konstant, wohingegen er bei der Gruppe der 6-MonatsAnwender zwischen Tag 14 und Woche 8 von $0.71 \pm 0.38$ auf $0.65 \pm 0.36$ weiterhin abfiel, um dann zwischen Woche 8 und Woche 16 kontinuierlich auf einen Mittelwert von $0.74 \pm 0.35$ anzusteigen. Die höchsten Werte in der Gruppe der sechsmonatigen Anwendung wurden nach Ablauf der 24 Wochen gemessen (0.93 \pm 0.43$)$. Im Vergleich hierzu wurde bei der Abschlussuntersuchung, d.h. eine Woche nach Ausgabe einer neuen Zahnbürste, ein geringerer Wert ermittelt (0.69 \pm 0.32$)$. Auch in Gruppe D (vierwöchiger Wechsel) wurde in Woche 25 ein durchschnittlich niedrigerer QHI erhoben als in Woche 24 (Abb. 15). 
Bürstentyp: Schall

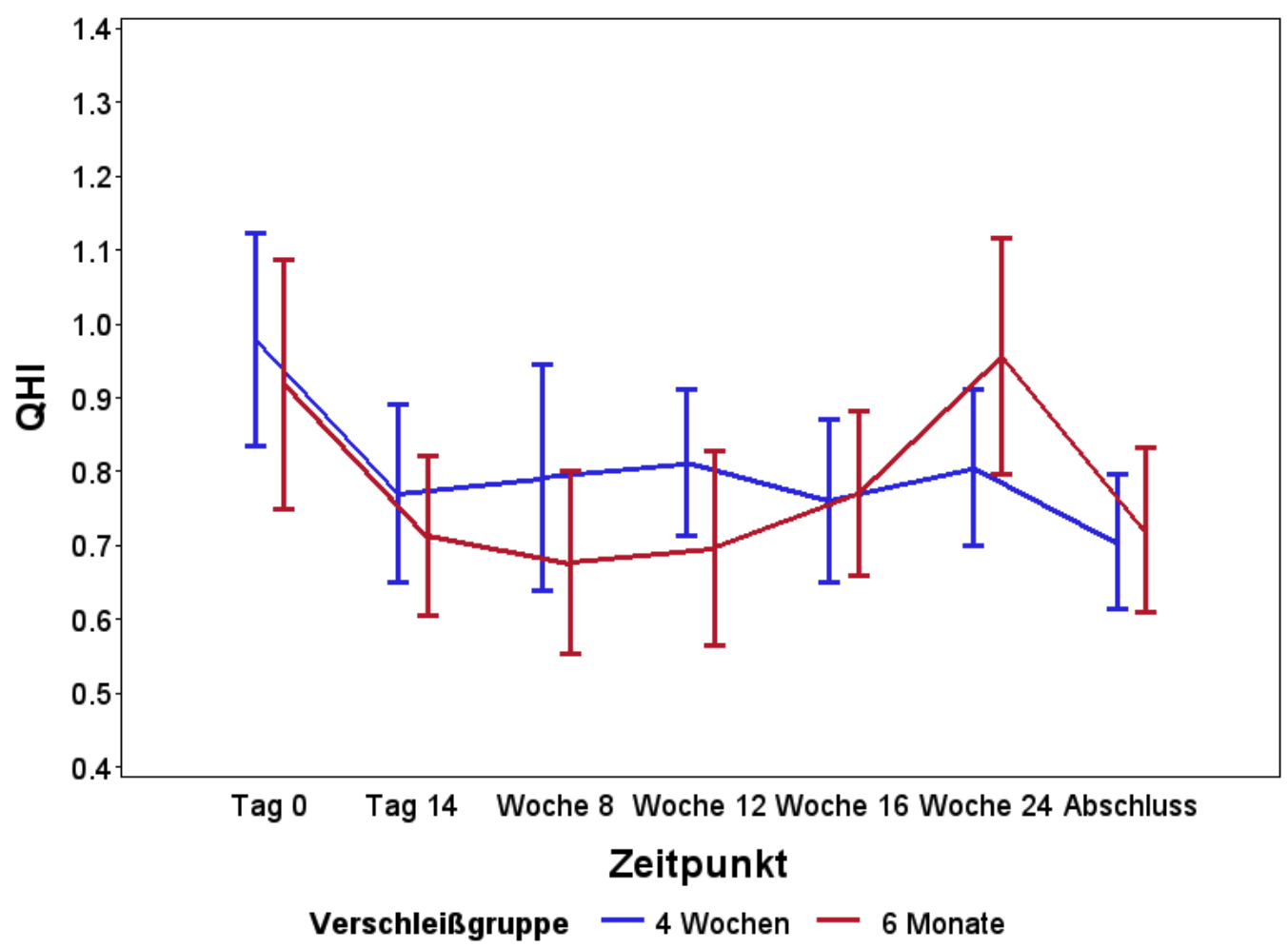

Abb. 15: Verlauf des QHI in den Gruppen C und D. Mittelwerte und 95\%-Konfidenzintervalle zu den jeweiligen Messzeitpunkten. Die zu den einzelnen Untersuchungszeitpunkten ermittelten Mittelwerte sind durch eine Linie verbunden.

\subsubsection{Analyse nach Verschleißgruppen}

Die statistische Auswertung zeigte einen signifikanten Unterschied in den Zeitverläufen der beiden Verschleißgruppen ( $\mathrm{p}=0.001)$. Daher erfolgte eine nach Verschleißgruppen getrennte Analyse.

\subsubsection{Verschleißgruppe: 4 Wochen}

Ein Einfluss des Bürstentyps (rotierend bzw. schallaktiv) wurde in dieser Verschleißgruppe nicht festgestellt $(\mathrm{p}=0.354)$. Daher erfolgte hier eine gemeinsame Auswertung. Der Faktor Zeitpunkt $(\mathrm{p}<.0001)$ und die Kovariable QHI baseline $(\mathrm{p}<.0001)$ hatten einen signifikanten Einfluss auf den zeitlichen Verlauf des QHI. Tabelle 4 zeigt die erhobenen QHI-Werte aller Untersuchungszeitpunkte (t1-t6) im Vergleich zur Baseline. Die Ergebnisse zeigen eine signifikante Verbesserung des Plaqueindex zu allen Zeitpunkten gegenüber Baseline $(\mathrm{p}<0.05)$ mit Ausnahme von Woche $12(\mathrm{p}=0.2559)$. 
Tabelle 4: Paarvergleiche gegen Baseline (Tag 0) für vierwöchigen Zahnbürstenwechsel

\begin{tabular}{|c|c|c|c|c|c|c|c|}
\hline Zeitpunkt & Zeitpunkt & $\begin{array}{c}\text { Schätz- } \\
\text { wert }\end{array}$ & $\begin{array}{l}\text { Standard- } \\
\text { abw. }\end{array}$ & $\begin{array}{l}\text { adjustier- } \\
\text { ter P-Wert }\end{array}$ & Alpha & adj. Lower & adj. Upper \\
\hline Tag 14 & Tag 0 & -0.2498 & 0.06263 & 0.0005 & 0.05 & -0.4034 & -0.09610 \\
\hline Woche 8 & Tag 0 & -0.2082 & 0.07711 & 0.0269 & 0.05 & -0.3973 & -0.01895 \\
\hline Woche 12 & Tag 0 & -0.1469 & 0.08820 & 0.2559 & 0.05 & -0.3633 & 0.06951 \\
\hline Woche 16 & Tag 0 & -0.1986 & 0.07612 & 0.0340 & 0.05 & -0.3854 & -0.01187 \\
\hline Woche 24 & Tag 0 & -0.1926 & 0.06323 & 0.0103 & 0.05 & -0.3478 & -0.03749 \\
\hline Woche 25 & Tag 0 & -0.2694 & 0.07710 & 0.0026 & 0.05 & -0.4586 & -0.08026 \\
\hline
\end{tabular}

Legende

„adj. Lower“/„adj. Upper“: adjustiertes Konfidenzintervall; untere/obere Grenze (siehe auch Tabelle 5, 6, 8, 9, $10,12,13)$

\subsubsection{Verschleißgruppe: 6 Monate}

In der Verschleißgruppe der 6-Monats-Verwender ließ sich eine signifikante Wechselwirkung zwischen Zeitpunkt und Bürstentyp nachweisen $(\mathrm{p}=0.014)$. Die Verschleißerscheinungen schienen demnach abhängig vom Bürstentyp aufzutreten. Es erfolgte daher eine getrennte Auswertung der Bürstentypen. Die Faktoren Zeitpunkt und QHI baseline beeinflussten den zeitlichen Verlauf des Plaqueindex bei beiden Bürstentypen signifikant ( $\mathrm{p}<.0001)$.

\section{Bürstentyp: Schall}

Tabelle 5 zeigt die QHI-Werte aller Untersuchungszeitpunkte im Vergleich zur Baseline für die schallaktive Testzahnbürste bei sechsmonatiger Anwendung. Eine signifikante Verbesserung bzw. Verschlechterung des Index im Vergleich zur Baseline konnte zu keinem Zeitpunkt festgestellt werden $(\mathrm{p}>0.05)$. 
Tabelle 5: Paarvergleiche gegen Baseline (Tag 0) für den sechsmonatigen Zahnbürstengebrauch

\begin{tabular}{|c|c|c|c|c|c|c|c|}
\hline Zeitpunkt & Zeitpunkt & $\begin{array}{c}\text { Schätz- } \\
\text { wert }\end{array}$ & $\begin{array}{l}\text { Standard- } \\
\text { abw. }\end{array}$ & $\begin{array}{l}\text { adjustier- } \\
\text { ter P-Wert }\end{array}$ & Alpha & adj. Lower & adj. Upper \\
\hline Tag 14 & Tag 0 & -0.2056 & 0.09302 & 0.1122 & 0.05 & -0.4602 & 0.04897 \\
\hline Woche 8 & Tag 0 & -0.2411 & 0.1100 & 0.1153 & 0.05 & -0.5421 & 0.05993 \\
\hline Woche 12 & Tag 0 & -0.2200 & 0.1227 & 0.2077 & 0.05 & -0.5557 & 0.1158 \\
\hline Woche 16 & $\operatorname{Tag} 0$ & -0.1481 & 0.1057 & 0.3557 & 0.05 & -0.4374 & 0.1412 \\
\hline Woche 24 & Tag 0 & 0.03750 & 0.1132 & 0.9738 & 0.05 & -0.2723 & 0.3473 \\
\hline Woche 25 & Tag 0 & -0.1971 & 0.1096 & 0.2061 & 0.05 & -0.4970 & 0.1028 \\
\hline
\end{tabular}

Bürstentyp: rotierend

Tabelle 6 stellt die QHI-Werte aller Untersuchungszeitpunkte im Vergleich zur Baseline für die rotierende Zahnbürste bei sechsmonatiger Anwendung dar. Eine signifikante Verbesserung bzw. Verschlechterung des Index im Vergleich zur Baseline konnte zu keinem Zeitpunkt festgestellt werden ( $\mathrm{p}>0.05)$.

Tabelle 6: Paarvergleiche gegen Baseline (Tag 0) für den sechsmonatigen Zahnbürstengebrauch

\begin{tabular}{|ccccccccc|}
\hline Zeitpunkt & Zeitpunkt & $\begin{array}{c}\text { Schätz- } \\
\text { wert }\end{array}$ & $\begin{array}{c}\text { Standard- } \\
\text { abw. }\end{array}$ & $\begin{array}{c}\text { adjustier- } \\
\text { ter P-Wert }\end{array}$ & Alpha & Adj. Lower & adj. Upper \\
Tag 14 & Tag 0 & -0.1731 & 0.1216 & 0.3170 & 0.05 & -0.4981 & 0.1519 \\
Woche 8 & Tag 0 & -0.1407 & 0.1464 & 0.5610 & 0.05 & -0.5319 & 0.2506 \\
Woche 12 & Tag 0 & -0.09717 & 0.1359 & 0.7262 & 0.05 & -0.4605 & 0.2662 \\
& & & & & & & \\
Woche 16 & Tag 0 & -0.06575 & 0.1650 & 0.9336 & 0.05 & -0.5067 & 0.3752 \\
Woche 24 & Tag 0 & 0.1712 & 0.1546 & 0.4736 & 0.05 & -0.2422 & 0.5845 \\
Woche 25 & Tag 0 & -0.2310 & 0.1257 & 0.1778 & 0.05 & -0.5671 & 0.1051 \\
& & & & & & & & \\
\hline
\end{tabular}




\subsection{Papillenblutungsindex (Gruppen A-D)}

Die Auswertung des PBI zeigte, unabhängig vom Bürstentyp, eine signifikante Wechselwir-

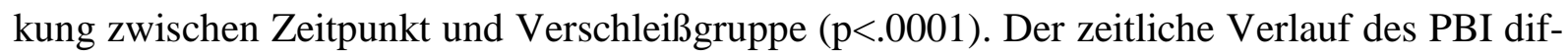
ferierte also je nach Verschleißgruppe (Tabelle 7).

Tabelle 7: Gruppenabhängige Mittelwerte $(\mathrm{MW}) \pm$ Standardabweichung (SD) und Medianwerte für den Parameter PBI zu allen Untersuchungszeitpunkten

\begin{tabular}{|ccccc|}
\hline Zeitpunkt & $\mathrm{A}$ & $\mathrm{B}$ & $\mathrm{C}$ & $\mathrm{D}$ \\
Baseline & $0.12 \pm 0.15$ & $0.27 \pm 0.17$ & $0.32 \pm 0.21$ & $0.28 \pm 0.22$ \\
& $(0.05)$ & $(0.24)$ & $(0.31)$ & $(0.25)$ \\
& $0.14 \pm 0.15$ & $0.24 \pm 0.19$ & $0.20 \pm 0.16$ & $0.20 \pm 0.14$ \\
Tag 14 & $(0.11)$ & $(0.17)$ & $(0.17)$ & $(0.17)$ \\
& $0.15 \pm 0.13$ & $0.28 \pm 0.17$ & $0.17 \pm 0.12$ & $0.23 \pm 0.19$ \\
Woche 8 & $(0.08)$ & $(0.28)$ & $(0.17)$ & $(0.21)$ \\
& $0.13 \pm 0.12$ & $0.25 \pm 0.19$ & $0.16 \pm 011$ & $0.21 \pm 0.09$ \\
Woche 12 & $(0.09)$ & $(0.18)$ & $(0.13)$ & $(0.17)$ \\
& $0.16 \pm 0.11$ & $0.25 \pm 0.16$ & $0.22 \pm 0.14$ & $0.22 \pm 0.14$ \\
Woche 16 & $(0.13)$ & $(0.19)$ & $(0.21)$ & $(0.20)$ \\
& & & $0.28 \pm 0.18$ & $0.22 \pm 0.11$ \\
Woche 24 & $0.31 \pm 0.16$ & $0.23 \pm 0.14$ & $(0.29)$ & $(0.21)$ \\
& $(0.25)$ & $(0.23)$ & $(0.25)$ & \\
& $0.12 \pm 0.13$ & $0.24 \pm 0.16$ & $0.20 \pm 0.15$ & $0.17 \pm 0.10$ \\
Woche 25 & $(0.07)$ & $(0.25)$ & $(0.21)$ & $(0.17)$ \\
& & & & \\
\hline
\end{tabular}

\subsubsection{Oral-B Professional Care}

Im Gegensatz zu der Verschleißgruppe des sechsmonatigen Zahnbürstengebrauchs (Gruppe A) verbesserten sich die Werte des PBI in der Gruppe des vierwöchigen Wechsels (Gruppe B) zwischen Tag 0 und Tag 14. Zwischen Tag 14 und Woche 16 blieb der PBI in beiden Gruppen auf einem ähnlichen Niveau. Während in Gruppe B über das gesamte Versuchsintervall hinweg keine erwähnenswerten Veränderungen zu verzeichnen waren, stiegen die ermittelten Werte in Gruppe A von Woche 16 an bis zum Ablauf der sechs Monate deutlich von $0.16 \pm 0.11$ auf $0.31 \pm 0.16$. Bei der Abschlussuntersuchung hingegen zeigte sich jedoch ein erheblich geringerer PBI (Abb. 16). 
Bürstentyp: rotierend

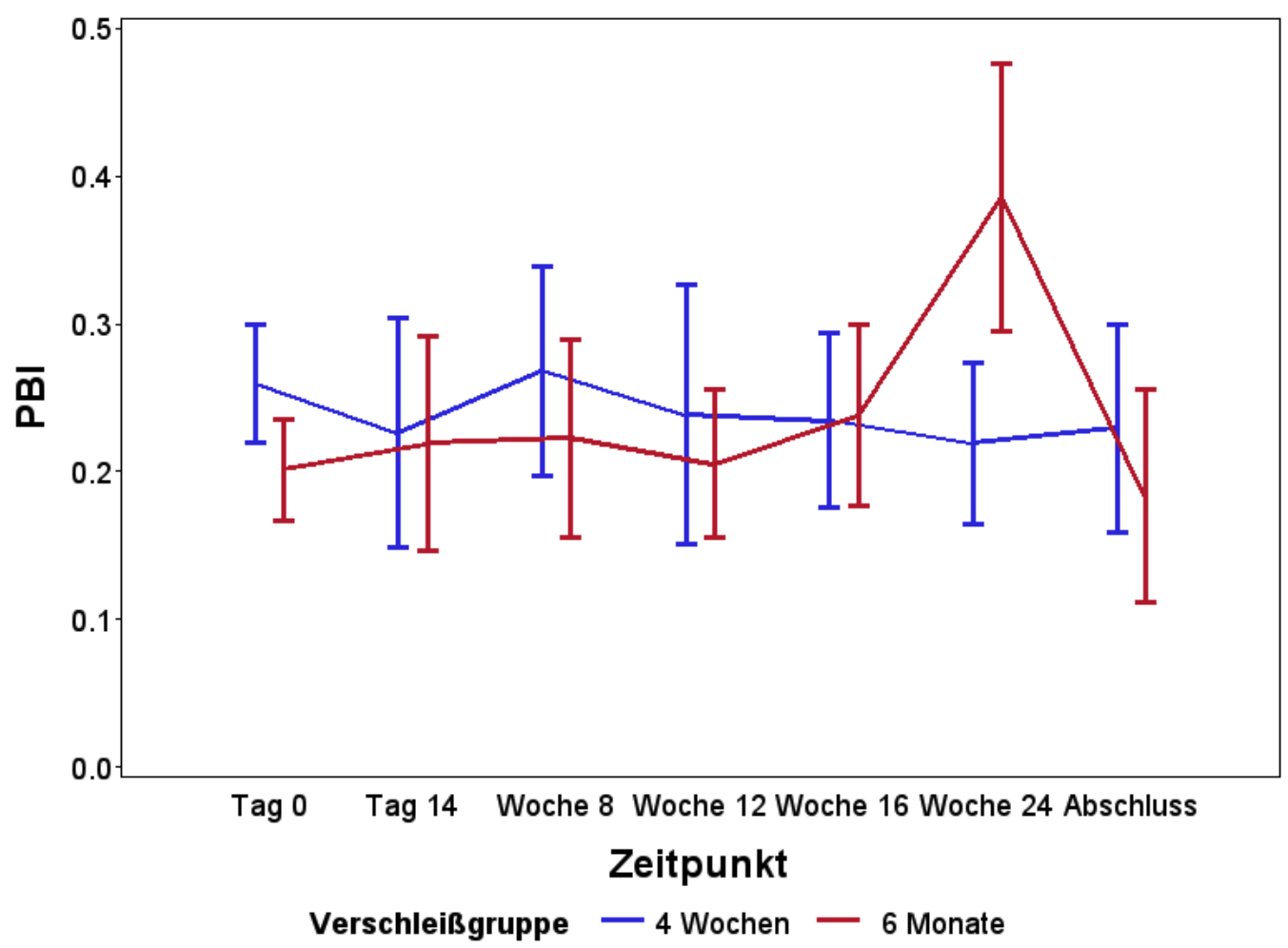

Abb. 16: Verlauf des PBI in den Gruppen A und B. Mittelwerte und 95\%-Konfidenzintervalle zu den jeweiligen Messzeitpunkten. Die zu den einzelnen Untersuchungszeitpunkten ermittelten Mittelwerte sind durch eine Linie verbunden.

\subsubsection{Philips Sonicare}

In beiden Verschleißgruppen konnte eine Verringerung des PBI am Tag 14 im Vergleich zu Tag 0 festgestellt werden. In der Gruppe der 6-Monats-Anwender blieb der PBI zwischen Tag 14 und Woche 12 auf nahezu konstantem Niveau, zwischen Woche 12 und Woche 24 stieg der Blutungsindex auf einen Mittelwert von 0.28 \pm 0.18 an, wohingegen er zwischen Woche 24 und 25 auf einen Mittelwert von 0.20 \pm 0.15 sank. Die Auswertung des PBI derjenigen Probanden, die alle vier Wochen eine neue Bürste benutzten, ließ zwischen Tag 14 und Woche 24 lediglich geringe Schwankungen erkennen; der Vergleich zwischen Woche 24 und 25 zeigte eine Verbesserung des PBI (Abb. 17). 
Bürstentyp: Schall

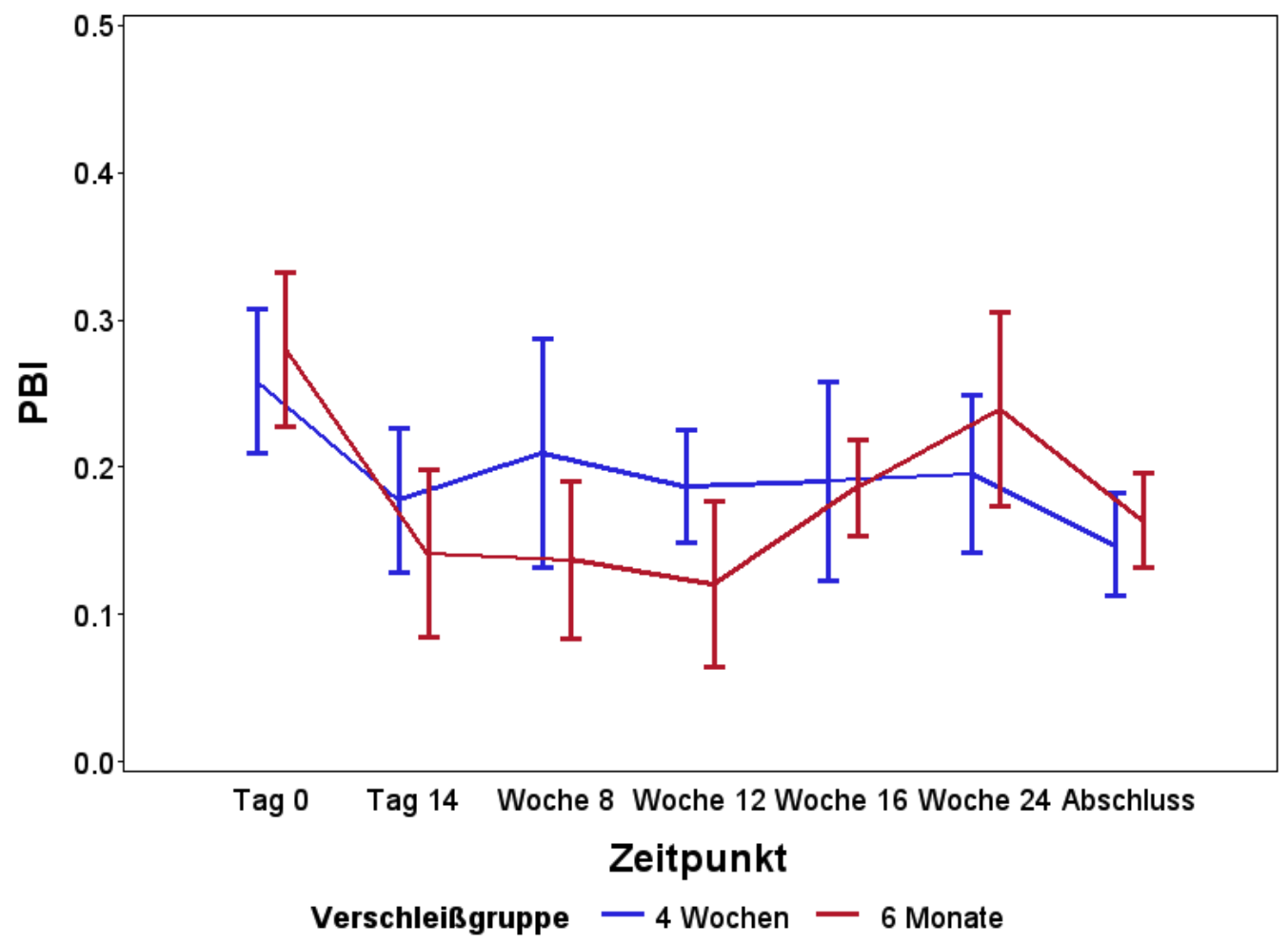

Abb. 17: Verlauf des PBI in den Gruppen C und D. Mittelwerte und 95\%-Konfidenzintervalle zu den jeweiligen Messzeitpunkten. Die zu den einzelnen Untersuchungszeitpunkten ermittelten Mittelwerte sind durch eine Linie verbunden.

\subsubsection{Analyse nach Verschleißgruppen}

\subsubsection{Verschleißgruppe: 4 Wochen}

Bei der vierwöchigen Zahnbürstenverwendung ergaben sich keine signifikanten Unterschiede zwischen der rotierend-oszillierenden und der schallaktiven Zahnbürste ( $p=0.1062)$. Daher erfolgt hier eine gemeinsame Auswertung beider Bürstentypen. Der Faktor Zeitpunkt beeinflusste den Verlauf des PBI signifikant ( $\mathrm{p}=0.0068$ ). Die Kovariable PBI baseline hatte einen signifikanten Einfluss auf den zeitlichen Verlauf des PBI ( $<<.0001)$.

In Tabelle 8 ist sind die Werte des PBI aller Untersuchungszeitpunkte im Vergleich zur Baseline dargestellt. Die Ergebnisse zeigten zu keinem Zeitpunkt signifikante Veränderungen des Index gegenüber Baseline $(\mathrm{p}<0.05)$. 
Tabelle 8: Paarvergleiche gegen Baseline (Tag 0) für den vierwöchigen Zahnbürstengebrauch

\begin{tabular}{|ccccccccc|}
\hline Zeitpunkt & Zeitpunkt & $\begin{array}{c}\text { Schätz- } \\
\text { wert }\end{array}$ & $\begin{array}{c}\text { Standard- } \\
\text { abw. }\end{array}$ & $\begin{array}{c}\text { adjustier- } \\
\text { ter P-Wert }\end{array}$ & Alpha & adj. Lower & adj. Upper \\
Tag 14 & Tag 0 & -0.05713 & 0.02686 & 0.1289 & 0.05 & -0.1252 & 0.01098 \\
Woche 8 & Tag 0 & -0.02023 & 0.03063 & 0.9400 & 0.05 & -0.09791 & 0.05744 \\
Woche 12 & Tag 0 & -0.04654 & 0.03268 & 0.4549 & 0.05 & -0.1294 & 0.03632 \\
Woche 16 & Tag 0 & -0.04643 & 0.02968 & 0.3681 & 0.05 & -0.1217 & 0.02885 \\
Woche 24 & Tag 0 & -0.05155 & 0.02838 & 0.2388 & 0.05 & -0.1235 & 0.02041 \\
Woche 25 & Tag 0 & -0.07035 & 0.02834 & 0.0571 & 0.05 & -0.1422 & 0.001527 \\
\hline
\end{tabular}

\subsubsection{Verschleißgruppe: 6 Monate}

Bei Verwendung derselben Zahnbürste über sechs Monate ließ sich eine signifikante Wechselwirkung zwischen Zeitpunkt und Bürstentyp nachweisen $(\mathrm{p}=0.0031)$. Die Verschleißerscheinungen schienen also abhängig vom verwendeten Bürstentyp aufzutreten. Daher erfolgte eine getrennte Auswertung der Bürstentypen. Statistisch signifikante Unterschiede zwischen Rauchern und Nichtrauchern wurden nicht festgestellt ( $p=0.9213)$.

\section{Bürstentyp: Schall}

Tabelle 9 zeigt die PBI-Werte aller Untersuchungszeitpunkte im Vergleich zur Baseline für die Schallzahnbürste bei 6-Monats-Anwendung. Signifikante Verbesserungen des Index im Vergleich zur Baseline ( $>00.05$ ) wurden zu allen Messzeitpunkten mit Ausnahme von Woche $24(\mathrm{p}=0.7254)$ festgestellt. 
Tabelle 9: Paarvergleiche gegen Baseline (Tag 0) für den sechsmonatigen Zahnbürstengebrauch

\begin{tabular}{|ccccccccc|}
\hline Zeitpunkt & Zeitpunkt & $\begin{array}{c}\text { Schätz- } \\
\text { wert }\end{array}$ & $\begin{array}{c}\text { Standard- } \\
\text { abw. }\end{array}$ & $\begin{array}{c}\text { adjustier- } \\
\text { ter P-Wert }\end{array}$ & Alpha & Adj. Lower & adj. Upper \\
Tag 14 & Tag 0 & -0.1389 & 0.03544 & 0.0132 & 0.05 & -0.2464 & -0.03136 \\
Woche 8 & Tag 0 & -0.1429 & 0.04323 & 0.0331 & 0.05 & -0.2741 & -0.01174 \\
Woche 12 & Tag 0 & -0.1595 & 0.04873 & 0.0348 & 0.05 & -0.3073 & -0.01160 \\
Woche 16 & Tag 0 & -0.09375 & 0.02973 & 0.0417 & 0.05 & -0.1840 & -0.00353 \\
Woche 24 & Tag 0 & -0.04050 & 0.03846 & 0.7254 & 0.05 & -0.1572 & 0.07620 \\
Woche 25 & Tag 0 & -0.1156 & 0.02559 & 0.0056 & 0.05 & -0.1932 & -0.03792 \\
\hline
\end{tabular}

\section{Bürstentyp: Rotierend}

Tabelle 10 zeigt die PBI-Werte aller Untersuchungszeitpunkte im Vergleich zur Baseline für die rotierend-oszillierende Bürste bei 6-Monats-Anwendung. In Woche 24 zeigte sich ein im Vergleich zur Baseline signifikant schlechterer Wert $(\mathrm{p}=0.0187)$. Zu allen anderen Messzeitpunkten waren die Veränderungen der PBI-Werte statistisch nicht signifikant ( $\mathrm{p}>0.05)$.

Tabelle 10: Paarvergleiche gegen Baseline (Tag 0) für den sechsmonatigen Zahnbürstengebrauch

\begin{tabular}{|ccccccccc|}
\hline Zeitpunkt & Zeitpunkt & Schätzwert & $\begin{array}{c}\text { Standard- } \\
\text { abw. }\end{array}$ & $\begin{array}{c}\text { adjustier- } \\
\text { ter P-Wert }\end{array}$ & Alpha & adj. Lower & adj. Upper \\
Tag 14 & Tag 0 & 0.01800 & 0.04243 & 0.9675 & 0.05 & -0.1026 & 0.1386 \\
Woche 8 & Tag 0 & 0.02175 & 0.04144 & 0.9300 & 0.05 & -0.09600 & 0.1395 \\
Woche 12 & Tag 0 & 0.004250 & 0.03197 & 0.9999 & 0.05 & -0.08658 & 0.09508 \\
Woche 16 & Tag 0 & 0.03692 & 0.04140 & 0.7110 & 0.05 & -0.08071 & 0.1545 \\
Woche 24 & Tag 0 & 0.1847 & 0.05299 & 0.0187 & 0.05 & 0.03409 & 0.3352 \\
Woche 25 & Tag 0 & -0.01717 & 0.04466 & 0.9781 & 0.05 & -0.1441 & 0.1097 \\
\hline
\end{tabular}




\subsection{Gingivaindex nach Löe und Silness (Gruppen A-D)}

Unabhängig vom verwendeten Zahnbürstentyp ließ sich eine signifikante Wechselwirkung zwischen Zeitpunkt und Verschleißgruppe nachweisen $(\mathrm{p}<.0001)$. Das heißt, der zeitliche Verlauf des QHI unterschied sich je nach Verschleißgruppe (Abb. 18 und Abb. 19, Tabelle 11).

Tabelle 11: Gruppenabhängige Mittelwerte (MW) \pm Standardabweichung (SD) und Medianwerte für den Parameter GI zu allen Untersuchungszeitpunkten

\begin{tabular}{|ccccc|}
\hline Zeitpunkt & $\mathrm{A}$ & $\mathrm{B}$ & $\mathrm{C}$ & $\mathrm{D}$ \\
& $0.59 \pm 0.26$ & $0.57 \pm 0.27$ & $0.73 \pm 0.43$ & $0.67 \pm 0.15$ \\
Baseline & $(0.67)$ & $(0.52)$ & $(0.67)$ & $(0.64)$ \\
& & & & \\
& $0.45 \pm 0.25$ & $0.57 \pm 0.23$ & $0.65 \pm 0.36$ & $0.57 \pm 0.18$ \\
Tag 14 & $(0.49)$ & $(0.54)$ & $(0.56)$ & $(0.53)$ \\
& $0.50 \pm 0.23$ & $0.56 \pm 0.25$ & $0.65 \pm 0.34$ & $0.66 \pm 0.22$ \\
Woche 8 & $(0.44)$ & $(0.58)$ & $(0.65)$ & $(0.61)$ \\
& $0.57 \pm 0.22$ & $0.58 \pm 0.23$ & $0.59 \pm 0.29$ & $0.66 \pm 0.25$ \\
Woche 12 & $(0.51)$ & $(0.55)$ & $(0.46)$ & $(0.59)$ \\
& $0.62 \pm 0.20$ & $0.58 \pm 0.21$ & $0.68 \pm 0.34$ & $0.65 \pm 0.23$ \\
Woche 16 & $(0.58)$ & $(0.54)$ & $(0.63)$ & $(0.59)$ \\
& & & & \\
Woche 24 & $0.83 \pm 0.26$ & $0.57 \pm 0.23$ & $0.88 \pm 0.33$ & $0.67 \pm 0.28$ \\
& $(0.78)$ & $(0.54)$ & $(0.91)$ & $(0.58)$ \\
& $0.47 \pm 0.22$ & $0.58 \pm 0.20$ & $0.67 \pm 0.33$ & $0.68 \pm 0.25$ \\
Woche 25 & $(0.47)$ & $(0.57)$ & $(0.64)$ & $(0.63)$ \\
& & & & \\
\hline
\end{tabular}

\subsubsection{Oral-B Professional Care}

Der zeitliche Verlauf des GI zeigte in Gruppe B (vierwöchiger Zahnbürstenwechsel) zu keinem Zeitpunkt nennenswerte Veränderungen. In der Gruppe der 6-Monats-Anwendung (Gruppe A) hingegen war zwischen Tag 0 und Tag 14 eine deutliche Verringerung des GI zu erkennen; vom ersten Kontrolltermin an stiegen die erhobenen Werte kontinuierlich bis zur vorletzten Untersuchung (Woche 24) von $0.45 \pm 0.25$ auf $0.83 \pm 0.26$. Zwischen Woche 24 und 25 gingen die Werte in der Gruppe der 6-Monats-Anwender deutlich zurück auf einen Mittelwert von 0.47 \pm 0.22 (Abb. 18). 
Bürstentyp: rotierend

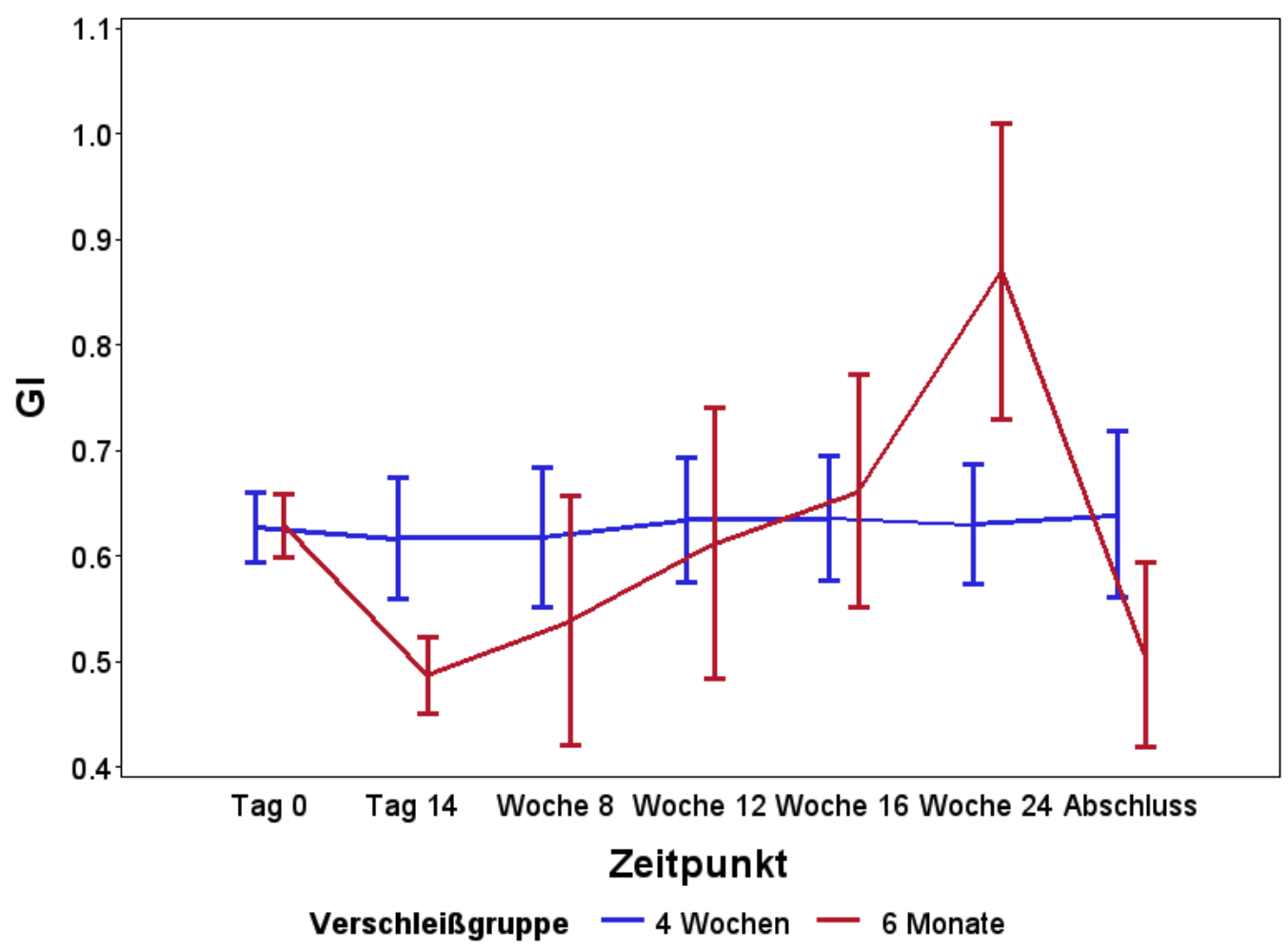

Abb. 18: Verlauf des GI in den Gruppen A und B. Mittelwerte und 95\%-Konfidenzintervalle zu den jeweiligen Messzeitpunkten. Die zu den einzelnen Untersuchungszeitpunkten ermittelten Mittelwerte sind durch eine Linie verbunden.

\subsubsection{Philips Sonicare}

Bei den Schallzahnbürsten zeigte sich in beiden Verschleißgruppen ein verbesserter GI an Tag 14 im Vergleich zu Tag 0 (Tabelle 11). Bei denjenigen Probanden, die alle vier Wochen eine neue Zahnbürste erhielten, stieg der GI zwischen Tag 14 und Woche 8 von $0.57 \pm 0.18$ auf $0.66 \pm 0.22$ an, blieb anschließend jedoch bis zum Abschluss der Untersuchung nahezu unverändert. In Gruppe C (Verwendung über sechs Monate) unterlag der GI zwischen Tag 14 und Woche 16 nur geringen Schwankungen. Zwischen Woche 16 und 24 verschlechterten sich die Mittelwerte deutlich von $0.68 \pm 0.34$ auf $0.88 \pm 0.33$, wohingegen sie sich bei der Abschlussuntersuchung wieder verbesserten (Abb. 19). 
Bürstentyp: Schall

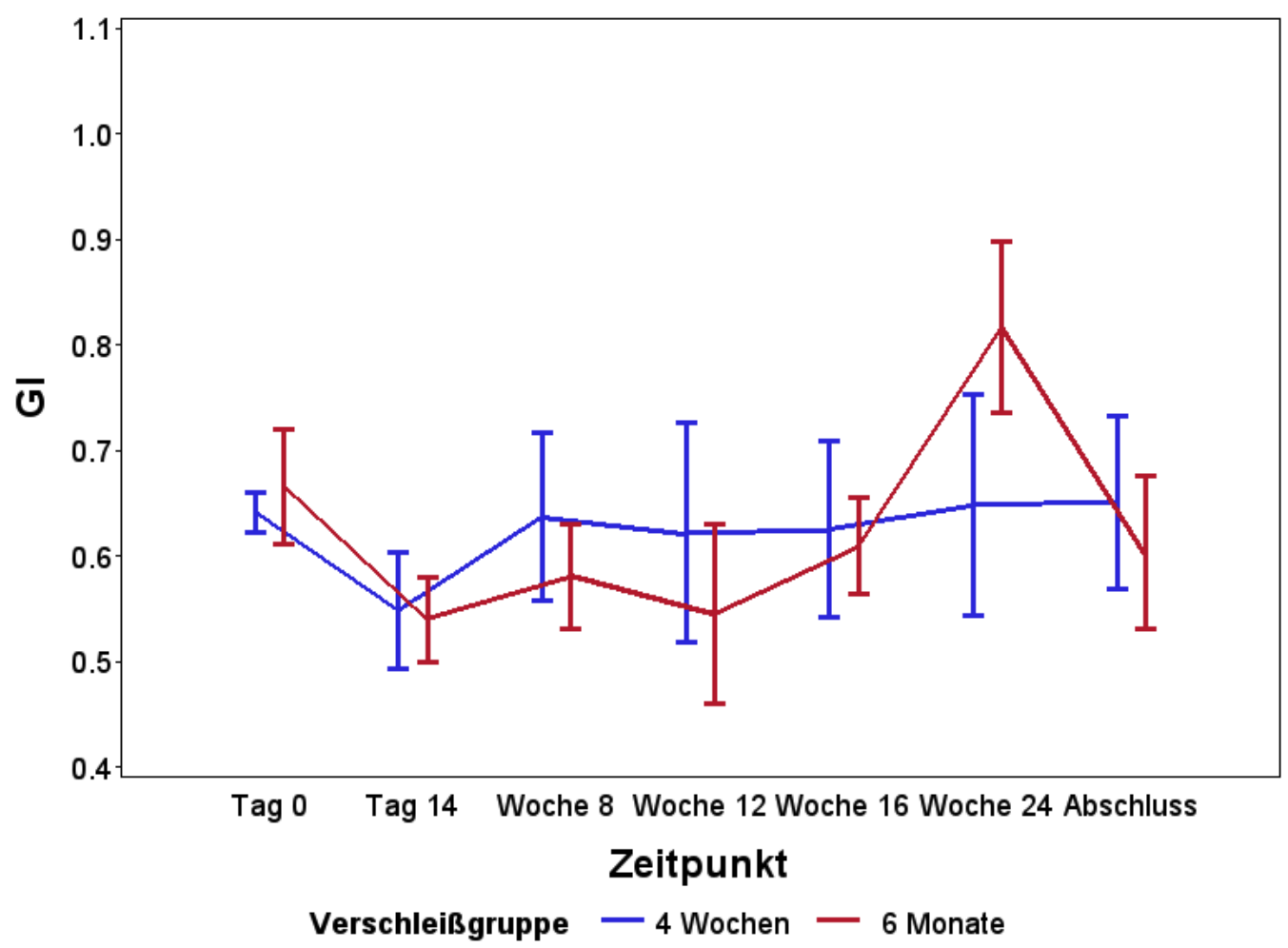

Abb. 19: Verlauf des GI in den Gruppen C und D. Mittelwerte und 95\%-Konfidenzintervalle zu den jeweiligen Messzeitpunkten. Die zu den einzelnen Untersuchungszeitpunkten ermittelten Mittelwerte sind durch eine Linie verbunden.

\subsubsection{Analyse nach Verschleißgruppen}

Die Auswertung ergab signifikante Unterschiede in den zeitlichen Verläufen des GI zwischen den 4-Wochen- und den 6-Monats-Anwendern ( $\mathrm{p}>.0001)$. Daher erfolgte eine nach Verschleißgruppen getrennte Analyse.

\subsubsection{Verschleißgruppe: 4 Wochen}

Innerhalb dieser Verschleißgruppe ließ sich eine signifikante Wechselwirkung zwischen Zeitpunkt und Bürstentyp nachweisen ( $\mathrm{p}=0.0392)$. In den nach Bürstentyp getrennten Subanalysen stellte sich allerdings kein signifikanter Zeiteffekt heraus (Schall: $p=0.08$, rotierend: $\mathrm{p}=0.89)$.

\subsubsection{Verschleißgruppe: 6 Monate}

Bei Verwendung derselben Zahnbürste über sechs Monate ließ sich eine signifikante Wechselwirkung zwischen Zeitpunkt und Bürstentyp nachweisen $(\mathrm{p}<.0001)$. Demnach traten die 
Verschleißerscheinungen abhängig vom verwendeten Bürstentyp auf, sodass eine separate Auswertung der Bürstentypen erfolgte.

\section{Bürstentyp: Schall}

Tabelle 12 stellt die GI-Werte aller Untersuchungszeitpunkte im Vergleich zur Baseline für die Schallzahnbürste bei 6-Monats-Anwendung dar. An Tag 14 zeigte sich ein im Vergleich zur Baseline signifikant besserer Wert $(\mathrm{p}=0.0018)$. Zu allen anderen Messzeitpunkten waren die Veränderungen der erhobenen Werte statistisch nicht signifikant ( $\mathrm{p}>0.05)$.

Tabelle 12: Paarvergleiche gegen Baseline (Tag 0) für den sechsmonatigen Zahnbürstengebrauch

\begin{tabular}{|ccccccccc|}
\hline Zeitpunkt & Zeitpunkt & Schätzwert & $\begin{array}{c}\text { Standard- } \\
\text { abw. }\end{array}$ & $\begin{array}{c}\text { adjustier- } \\
\text { ter P-Wert }\end{array}$ & Alpha & adj. Lower & adj. Upper \\
Tag 14 & Tag 0 & -0.1246 & 0.02374 & 0.0018 & 0.05 & -0.1945 & -0.05475 \\
Woche 8 & Tag 0 & -0.08500 & 0.03805 & 0.1458 & 0.05 & -0.1970 & 0.02696 \\
Woche 12 & Tag 0 & -0.1186 & 0.06360 & 0.2479 & 0.05 & -0.3058 & 0.06848 \\
Woche 16 & Tag 0 & -0.05625 & 0.03775 & 0.4083 & 0.05 & -0.1673 & 0.05481 \\
Woche 24 & Tag 0 & 0.1505 & 0.05622 & 0.0749 & 0.05 & -0.01490 & 0.3159 \\
Woche 25 & Tag 0 & -0.06283 & 0.05087 & 0.5524 & 0.05 & -0.2125 & 0.08684 \\
\hline
\end{tabular}

\section{Bürstentyp: Rotierend}

Die GI-Werte aller Untersuchungszeitpunkte im Vergleich zur Baseline für die rotierendoszillierende Zahnbürste bei sechsmonatiger Anwendung sind in Tabelle 13 dargestellt. Eine signifikante Verbesserung des Wertes zeigte sich an Tag $14(\mathrm{p}=0.0003)$. Nach Ablauf der 24 Wochen ergab sich ein signifikant schlechterer GI $(p=0.0341)$. 
Tabelle 13: Paarvergleiche gegen Baseline (Tag 0) für den sechsmonatigen Zahnbürstengebrauch

\begin{tabular}{|c|c|c|c|c|c|c|c|}
\hline Zeitpunkt & Zeitpunkt & $\begin{array}{l}\text { Schätz- } \\
\text { wert }\end{array}$ & $\begin{array}{c}\text { Standard- } \\
\text { abw. }\end{array}$ & $\begin{array}{l}\text { adjustier- } \\
\text { ter P-Wert }\end{array}$ & Alpha & Adj. Lower & adj. Upper \\
\hline Tag 14 & Tag 0 & -0.1416 & 0.02091 & 0.0003 & 0.05 & -0.2021 & -0.08103 \\
\hline Woche 8 & Tag 0 & -0.08975 & 0.06769 & 0.4805 & 0.05 & -0.2858 & 0.1063 \\
\hline Woche 12 & Tag 0 & -0.01700 & 0.07443 & 0.9978 & 0.05 & -0.2326 & 0.1986 \\
\hline Woche 16 & $\operatorname{Tag} 0$ & 0.03300 & 0.06529 & 0.9435 & 0.05 & -0.1561 & 0.2221 \\
\hline Woche 24 & Tag 0 & 0.2418 & 0.07693 & 0.0341 & 0.05 & 0.01903 & 0.4646 \\
\hline Woche 25 & Tag 0 & -0.1216 & 0.05098 & 0.1101 & 0.05 & -0.2692 & 0.02606 \\
\hline
\end{tabular}

\subsection{Zusammenfassung der Ergebnisse (Gruppen A-D)}

Bei allen drei erhobenen Indizes ergaben sich in der Gruppe der 6-Monats-Anwender signifikant schlechtere Werte als bei Testpersonen, die alle vier Wochen eine neue Zahnbürste verwendeten.

Beim Parameter Plaquebefall (QHI) zeigte sich in der Verschleißgruppe des vierwöchigen Zahnbürstenwechsels eine in etwa konstante Verbesserung des Plaqueindex gegenüber dem Baseline-Wert über den gesamten Zeitraum bei beiden Zahnbürstentypen (Abb. 14 und Abb. 15). Ein Einfluss des Bürstentyps (rotierend-oszillierend bzw. schallaktiv) wurde in dieser Verschleißgruppe nicht festgestellt $(\mathrm{p}=0.354)$. Dagegen ließ sich in der Gruppe der 6-MonatsAnwender ein im Vergleich zur Baseline niedrigerer QHI lediglich über einen Zeitraum von vier Monaten feststellen. Ab Woche 16, besonders deutlich jedoch nach sechsmonatiger Anwendung derselben Zahnbürste, war schließlich ein erhöhter Plaquebefall, besonders in Gruppe A (rotierend-oszillierende Zahnbürste), zu beobachten. Bei Anwendung der rotierendoszillierenden Zahnbürste ergaben sich, verglichen mit der Baseline, in Woche 24 höhere Werte als bei Verwendung der Schallzahnbürste.

Ähnlich gestaltete sich der zeitliche Verlauf des PBI: Während sich die erhobenen Werte derjenigen Probanden, die über sechs Monate mit derselben Zahnbürste putzten, über die Zeit deutlich gegenüber Baseline verschlechterten, unterlagen sie in der Gruppe des vierwöchigen Zahnbürstenwechsels unabhängig vom verwendeten Bürstentyp lediglich geringen Schwankungen (Abb. 16 und Abb. 17). In der Gruppe der 6-Monats-Anwender ließ sich eine signifikante Wechselwirkung zwischen Zeitpunkt und Bürstentyp nachweisen ( $p=0.0031$ ). 
Folglich traten die Verschleißerscheinungen abhängig vom verwendeten Bürstentyp auf: Während sich der PBI bei Probanden, die die rotierend-oszillierende Bürste benutzten, nach Ablauf der 24 Wochen signifikant verschlechterte ( $\mathrm{p}=0.0187)$, unterschied sich der Index bei Anwendern der Schallzahnbürste nach sechs Monaten nicht signifikant von der Baseline $(p=0.7254)$. Im Gegensatz zu den Anwendern der rotierend-oszillierenden Bürste wurde bei den 6-Monats-Anwendern der schallaktiven Zahnbürste eine Verbesserung des PBI im Vergleich zur Baseline $(0.32 \pm 0.21)$ sogar noch in Woche $24(0.28 \pm 0.18)$ beobachtet.

Die Auswertung des GI zeigte ebenfalls, dass sich der zeitliche Verlauf in der Gruppe der 4-Wochen-Anwender signifikant vom Verlauf in der Gruppe des sechsmonatigen Gebrauchs unterschied. Der Index verschlechterte sich bei den 6-Monats-Anwendern in Woche 24 im Vergleich zur Baseline bei beiden Zahnbürstentypen. Dagegen blieben die Werte beim vierwöchigen Zahnbürstenwechsel über den gesamten Zeitraum stabil (Abb. 18 und Abb. 19). Zwar ergab die Auswertung innerhalb der 4-Wochen-Verschleißgruppe eine signifikante Wechselwirkung zwischen Zeitpunkt und Bürstentyp ( $\mathrm{p}=0.0392)$, jedoch stellte sich in den nach Bürs-tentyp getrennten Subanalysen kein signifikanter Zeiteffekt heraus (Schall: $\mathrm{p}=0.0830$, rotierend $\mathrm{p}=0.8872$ ). Bei Verwendung derselben Zahnbürste über sechs Monate ließ sich eine signifikante Wechselwirkung zwischen Zeitpunkt und Bürstentyp nachweisen ( $\mathrm{p}<.0001)$. Die Verschleißerscheinungen schienen abhängig vom verwendeten Bürstentyp aufzutreten: Während sich der GI bei Anwendern der rotierend-oszillierenden Bürste nach Ablauf der 24 Wochen signifikant verschlechterte $(\mathrm{p}=0.0341)$, zeigte sich bei Verwendern der Schallzahnbürste nach sechs Monaten keine signifikante Verschlechterung des Index $(p=0.0749)$.

\subsection{Ergebnisse der elektrisch unterstützten Handzahnbürsten (Gruppen E und F)}

Aufgrund der limitierten Batterielaufzeit der elektrisch unterstützten Handzahnbürsten war eine Studiendauer von sechs Monaten in den Gruppen E und F nicht möglich. Ein unmittelbarer Vergleich mit den beiden anderen Testzahnbürsten erschien daher wenig sinnvoll, sodass die Ergebnisse der untersuchten Handzahnbürsten gesondert aufgeführt wurden. Auf eine statistische Auswertung wie sie in den Gruppen A-D erfolgte, wurde aus den oben genannten Gründen ebenfalls verzichtet. Die Ergebnisse der in den Gruppen E und F untersuchten Zahnbürsten werden daher deskriptiv aufgeführt. 


\subsubsection{QHI}

\subsubsection{Gruppe E}

Der niedrigste QHI wurde bei der Eingangsuntersuchung (t0) gemessen (0.67 \pm 0.45 ), der höchste Wert ergab sich bei derjenigen Untersuchung, die unmittelbar nach Ablauf der Batterielaufzeit stattfand $(0.87 \pm 0.35)$. Eine Woche nach diesem Kontrolltermin, das heißt nach Ausgabe einer neuen Zahnbürste, wurde ein vergleichsweise geringerer QHI erhoben (0.71 \pm 0.32$)$. Bei lediglich zwei der insgesamt elf Testpersonen dieser Gruppe konnten die Mundhygieneindizes auch noch nach Woche 12 erfasst werden, da bei allen anderen Testzahnbürsten der Akku bereits zu einem früheren Zeitpunkt aufgebraucht war. Daher wurden in den Tabellen 14-16 für diesen Untersuchungszeitpunkt die Mittelwerte (bzw. Standardabweichungen und Medianwerte) nicht bewertet.

\subsubsection{Gruppe F}

Bei Probanden, die alle vier Wochen eine neue Bürste erhielten, ergab sich der niedrigste Mittelwert $(0.77 \pm 0.34)$ bei der Abschlussuntersuchung; der höchste Wert wurde in Woche 8 ermittelt $(0.86 \pm 0.37)$.

Tabelle 14: Gruppenabhängige Mittelwerte (MW) \pm Standardabweichung (SD) und Medianwerte für den Parameter QHI zu allen Messzeitpunkten

\begin{tabular}{|ccc|}
\hline Zeitpunkt & Gruppe E & Gruppe F \\
Baseline & $0.67 \pm 0.45$ & $0.85 \pm 0.50$ \\
& $(0.55)$ & $(0.67)$ \\
Tag 14 & $0.69 \pm 0.36$ & $0.79 \pm 0.43$ \\
& $(0.72)$ & $(0.72)$ \\
Woche 8 & $0.84 \pm 0.44$ & $0.86 \pm 0.37$ \\
& $(0.64)$ & $(0.89)$ \\
Woche 12 & nicht bewertet & $0.83 \pm 0.35$ \\
Kontrolle nach Ende der & $0.87 \pm 0.35$ & $(0.88)$ \\
Akkulaufzeit & $(0.93)$ & nicht bewertet \\
& & $0.80 \pm 0.38$ \\
Woche 16 & nicht bewertet & $(0.81)$ \\
& $0.71 \pm 0.32$ & $0.77 \pm 0.34$ \\
Abschlusskontrolle & $(0.70)$ & $(0.82)$ \\
& &
\end{tabular}




\subsubsection{PBI}

\subsubsection{Gruppe E}

Der Mittelwert des PBI verbesserte sich zwischen Tag 0 (0.22 \pm 0.08$)$ und Tag $14(0.16 \pm 0.13)$. Der schlechteste Wert innerhalb dieser Gruppe wurde an jenem Termin ermittelt, der unmit-

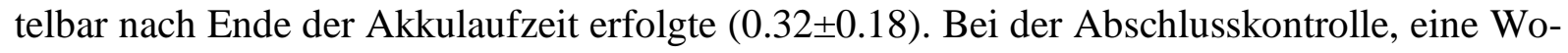
che nach Ausgabe einer neuen Bürste, ergab sich ein vergleichsweise geringerer Mittelwert von $0.21 \pm 0.13$ (Tabelle 15).

\subsubsection{Gruppe F}

In der Gruppe der 4-Wochen-Anwender zeigte sich an Tag 14 ein verbesserter Mittelwert $(0.23 \pm 0,24)$ im Vergleich zu Tag $0(0.36 \pm 0.35)$. Der höchste Wert wurde in Woche 8 gemessen (0.29 \pm 0.30$)$, der niedrigste Mittelwert zeigte sich bei der Abschlussuntersuchung

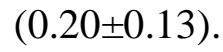

Tabelle 15: Gruppenabhängige Mittelwerte (MW) \pm Standardabweichung (SD) und Medianwerte für den Parameter PBI zu allen Messzeitpunkten

\begin{tabular}{|ccc|}
\hline Zeitpunkt & Gruppe E & Gruppe F \\
Baseline & $0.22 \pm 0.08$ & $0.36 \pm 0.35$ \\
& $(0.24)$ & $(0.27)$ \\
Tag 14 & $0.16 \pm 0.13$ & $0.23 \pm 0.24$ \\
& $(0.19)$ & $(0.17)$ \\
Woche 8 & $0.21 \pm 0.13$ & $0.29 \pm 0.30$ \\
& $(0.17)$ & $(0.21)$ \\
Woche 12 & & $0.21 \pm 0.17$ \\
& nicht bewertet & $(0.17)$ \\
Kontrolle nach Ende der & $0.32 \pm 0.18$ & nicht bewertet \\
Akkulaufzeit & $(0.33)$ & $0.27 \pm 0.34$ \\
& & $(0.15)$ \\
Woche 16 & nicht bewertet & $0.20 \pm 0.24$ \\
& $0.21 \pm 0.13$ & $(0.13)$ \\
Abschlusskontrolle & $(0.21)$ & \\
& &
\end{tabular}




\subsubsection{GI}

\subsubsection{Gruppe E}

Die Mittelwerte des Gingivaindex bewegten sich innerhalb des Kontrollintervalls zwischen 0.54 \pm 0.29 (Tag 14) und 0.69 \pm 0.29 (Untersuchung nach Ende der Akkulaufzeit). Nach Ausgabe einer neuen Zahnbürste verbesserte sich der Mittelwert von $0.69 \pm 0.29$ auf $0.58 \pm 0.30$ bei der Abschlussuntersuchung (Tabelle 16).

\subsubsection{Gruppe F}

Bei Testpersonen, die alle vier Wochen eine neue Zahnbürste verwendeten, variierten die er-

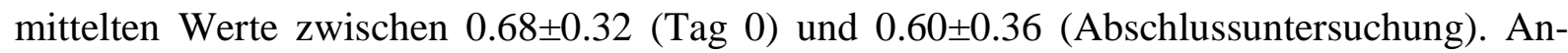
hand des an Tag 14 erhobenen Wertes (0.62 \pm 0.32$)$ konnte eine Verbesserung des GI im Vergleich zur Eingangsuntersuchung (Tag 0) festgestellt werden.

Tabelle 16: Gruppenabhängige Mittelwerte (MW) \pm Standardabweichung (SD) und Medianwerte für den Parameter GI zu allen Messzeitpunkten

\begin{tabular}{|ccc|}
\hline Zeitpunkt & Gruppe E & Gruppe F \\
Baseline & $0.56 \pm 0.32$ & $0.68 \pm 0.37$ \\
& $(0.38)$ & $(0.62)$ \\
Tag 14 & $0.54 \pm 0.29$ & $0.62 \pm 0.32$ \\
& $(0.41)$ & $(0.54)$ \\
Woche 8 & $0.59 \pm 0.28$ & $0.63 \pm 0.30$ \\
& $(0.46)$ & $(0.54)$ \\
Woche 12 & & $0.66 \pm 0.33$ \\
& nicht bewertet & $(0.66)$ \\
Kontrolle nach Ende der & $0.69 \pm 0.29$ & nicht bewertet \\
Akkulaufzeit & $(0.56)$ & $0.60 \pm 0.34$ \\
& & $(0.52)$ \\
Woche 16 & nicht bewertet & $0.60 \pm 0.36$ \\
& $0.58 \pm 0.30$ & $(0.49)$ \\
Abschlusskontrolle & $(0.46)$ & \\
& &
\end{tabular}




\subsubsection{Durchschnittliche Batterielaufzeiten}

Die durchschnittliche Batterielaufzeit der getesteten Handzahnbürsten betrug 84.45 \pm 9.24 Tage.

\subsubsection{Zusammenfassung der Ergebnisse}

Sowohl beim Plaqueindex, als auch bei den beiden Gingivaindizes zeigten sich die höchsten Mittelwerte jeweils an jenem Kontrolltermin, der unmittelbar nach dem Ende der Batterielaufzeit der getesteten Zahnbürsten stattfand. Mit Ausnahme des QHI in Gruppe E verbesserten sich die Mittelwerte aller drei Mundhygieneindizes zwischen der Eingangsuntersuchung (Tag 0) und Tag 14 (Tabelle 14-16). Des Weiteren stellten sich die während der Abschlusskontrolle ermittelten Werte niedriger dar als die Werte, die beim letzten Kontrolltermin erhoben wurden. Die Ausnahme bildet hier allerdings der GI in der Gruppe der 4-WochenAnwender. 


\section{Diskussion}

Gegenstand der vorliegenden Arbeit war die Untersuchung des Einflusses der Gebrauchsdauer verschiedener elektrischer Zahnbürsten auf die mechanische Plaquekontrolle und den gingivalen Zustand. Hierbei sollte die Hypothese überprüft werden, dass die Gebrauchsdauer einer Zahnbürste mit ihrer Reinigungswirkung korreliert.

\subsection{Studiendesign}

\subsubsection{Paralleldesign}

Generell finden bei Zahnbürstenstudien zwei verschiedene Studiendesigns Anwendung. Bei dem in dieser Studie durchgeführten Paralleldesign verwendeten die Probanden zeitlich parallel nur jeweils eine Zahnbürste. Dieses Vorgehen bietet den Vorteil einer verkürzten Studienlaufzeit. Da die Dauer der vorliegenden Untersuchung mit mehr als 24 Wochen ohnehin sehr lang war, kam eine Durchführung im Cross-over-Design nicht in Frage, weil sich hierdurch eine deutliche Verlängerung der Studienlaufzeit ergeben hätte. Um statistisch repräsentative Ergebnisse zu erzielen, ist beim Paralleldesign zwar eine höhere Probandenanzahl erforderlich, jedoch können mögliche Wechselwirkungen, welche bedingt durch die einzelnen Testintervalle beim Cross-over-Design auftreten können, ausgeschlossen werden (CHILTON und FLEISS 1986). Des Weiteren besteht bei Studien, die im Paralleldesign durchgeführt werden, kein Risiko, dass die Testergebnisse durch bewusste oder unbewusste Begünstigung einer der verwendeten Zahnbürsten seitens der Probanden verfälscht werden, da jeder Studienteilnehmer nur jeweils einen Zahnbürstentyp anwendet. Auch Carry-over-Effekte wie Motivationsschwankungen, Neuheits- oder Trainingseffekte und Ermüdung sind beim Paralleldesign nicht zu befürchten (BORTZ 2005).

\subsubsection{Reliabilität}

Zur Gewährleistung der Reliabilität wurden alle Messungen untersucherblind von derselben Zahnärztin vorgenommen. Um die einzelnen Untersuchungen nicht durch eventuelle eigene Erwartungen zu beeinflussen, wurde die Einteilung der Probanden in die jeweiligen Gruppen durch eine zweite Person mit Hilfe eines Losverfahrens durchgeführt. Die Zuteilung der neuen Zahn- bzw. Aufsteckbürsten erfolgte ebenfalls durch eine zweite Person. Über welchen Zeitraum ein Proband die ihm zugewiesene Zahnbürste verwendete und um welche Bürste es sich handelte, war der Untersucherin somit zu keinem Zeitpunkt der Studie bekannt. 
Die möglichst präzise Erhebung der Indizes und die daraus resultierende Reproduzierbarkeit der Ergebnisse wurden durch eine Kalibrierung der Untersucherin im Vorfeld der Studie gesichert.

\subsubsection{Studiendauer/Kontrollintervall}

Die Studiendauer der meisten Zahnbürstenstudien beträgt zwischen 30 und 90 Tagen (GLAZE and WADE 1986, SFORZA et al. 2000, CONFORTI et al. 2001, CRONIN et al. 2001, TAN und DALY 2002, HOGAN et al. 2007); je nach Zielsetzung der Untersuchung kann jedoch eine kürzere oder längere Testphase indiziert sein. Um festzustellen, ob die Reinigungswirkung einer Zahnbürste, die länger als drei Monate benutzt wurde, nachlässt und um zu überprüfen, ob ein Zahnbürstenwechsel nach etwa drei Monaten, wie im Allgemeinen empfohlen, sinnvoll erscheint, wurde für diese Studie ein Zeitraum von mehr als 12 Wochen festgelegt. Die mit 25 Wochen erheblich längere Studiendauer begründet sich darin, dass in der vorliegenden Arbeit zusätzlich untersucht werden sollte, inwiefern sich die Reinigungswirkung der verschiedenen Zahnbürsten nach Ablauf der empfohlenen drei Monate, nämlich nach vier- bzw. sechsmonatiger Gebrauchsdauer verändert.

Der erste Kontrolltermin fand 14 Tage nach der zu Studienbeginn durchgeführten professionellen Zahnreinigung statt, da sich frühestens innerhalb dieser Zeitspanne eine Gingivitis etablieren kann (LÖE et al. 1965). Die Erhebung eines Gingivaindex zu einem früheren Zeitpunkt wäre deshalb wenig aussagefähig gewesen. Um die Anzahl der jeweiligen Kontrolltermine auf ein sinnvolles Maß zu reduzieren, fanden diese nicht vierwöchentlich, sondern lediglich 8, 12, 16 und 24 Wochen nach Studienbeginn statt. Der letzte Kontrolltermin, eine Woche nach Ablauf der 24 Wochen, diente zur Erfassung der Mundhygienesituation nach der einwöchigen Verwendung einer neuen Zahnbürste. Hierbei sollte überprüft werden, welchen Veränderungen die Plaque- und Gingivaindizes derjenigen Probanden unterlagen, die über die gesamte Studiendauer dieselbe Zahnbürste benutzten.

\subsubsection{Probandenanzahl}

Für eine valide statistische Auswertung ist eine möglichst hohe Anzahl an Probanden von Vorteil. Um aussagekräftige Ergebnisse zu erlangen, wurde im Falle dieser Studie eine Probandenanzahl von 15 Personen in jeder der sechs Gruppen angestrebt. Schließlich konnten die erhobenen Daten von 71 Probanden statistisch ausgewertet werden. Die Teilnehmerzahl anderer vergleichender Zahnbürstenstudien variiert zwischen 15 (NIEMI 1987) und 193 
(SHARMA et al.1994). Nach den Richtlinien der American Dental Association (ADA 1998) sollte die Mindestgruppengröße klinischer Studien 25 Probanden betragen, jedoch bleibt zu berücksichtigen, dass dies die erste Untersuchung ist, die den Einfluss der Gebrauchsdauer von Zahnbürsten auf die Effizienz der Plaquereduktion und den Entzündungsgrad der Gingiva über einen Zeitraum von 25 Wochen untersuchte.

\subsubsection{Probandenauswahl}

Mit Hilfe eines Anamnesebogens und der Erhebung eines klinischen Befundes wurden Patienten mit Allgemeinerkrankungen, bestimmter Medikation, Parodontitiden, festsitzenden kieferorthopädischen Apparaturen oder herausnehmbarem Zahnersatz von der Studienteilnahme ausgeschlossen. Auch Patienten mit einem Zahnbestand von weniger als 20 Zähnen nahmen nicht an der Studie teil (vgl. Kap. 3.1). Somit wurden eine Vielzahl möglicher beeinflussender Faktoren bereits im Vorfeld der Untersuchung eliminiert und die Validität der Untersuchung gesichert.

Wie für zahlreiche andere Zahnbürstenstudien (GLAZE und WADE 1986, VAN DER WEIJDEN et al. 1998) wurden auch in der vorliegenden Untersuchung Zahnmedizinstudenten und zahnärztliches Personal als Probanden rekrutiert. Da dieser Personenkreis eine relativ homogene Gruppe mit konstanten Zahnpflegegewohnheiten darstellt, die über ein gewisses Maß an Fachwissen verfügt, kann davon ausgegangen werden, dass der Einfluss individueller, probandenbedingter Faktoren relativ niedrig gehalten werden konnte und die Testergebnisse auf diese Weise nur minimal beeinflusst wurden.

\subsubsection{Zahnputzdauer und Mundhygieneinstruktion}

Allen Studienteilnehmern wurde eine Putzdauer von zwei Minuten bei zweimaligem Zähneputzen pro Tag vorgegeben, um standardisierte Voraussetzungen zu schaffen. Während die American Dental Association eine Zahnputzdauer von drei Minuten empfiehlt (ADA 1998), stellte sich der Hauptputzeffekt in Studien von VAN DER WEIJDEN et al. schon nach 30 Sekunden pro Quadrant ein (VAN DER WEIJDEN et al. 1993 und 1996a). Da sowohl die zu untersuchende schallaktive als auch die rotierend-oszillierende Zahnbürste über integrierte Timer verfügen, die pro Quadrant ebenfalls eine Putzdauer von 30 Sekunden vorsehen, entschied man sich bei den elektrisch unterstützten Handzahnbürsten ebenfalls für eine Putzzeit von zwei Minuten. In allen Gruppen wurden gleichermaßen qualifizierte Mundhygieneinstruktionen durch die Untersucherin erteilt; die Probanden wurden angewiesen, während der 
gesamten Studiendauer keine weiteren Mundhygienehilfsmittel wie Zahnseide oder Interdentalraumbürsten zu verwenden.

\subsubsection{Randomisierung}

Die Verteilung der Studienteilnehmer auf die Versuchsgruppen erfolgte anhand des QHI, um sicherzustellen, dass das Mundhygieneniveau zu Beginn der Studie in allen Gruppen ähnlich war. Die Probanden wurden anhand der in der Screening-Untersuchung gemessenen QHIWerte in drei Mundhygiene-Kategorien (gut, mittelmäßig, schlecht) eingestuft und anschließend per Losverfahren gleichmäßig auf die sechs Gruppen A-F verteilt. Außerdem wurde eine Randomisierung nach Rechts- und Linkshändigkeit vorgenommen.

\subsection{Material}

\subsubsection{Testzahnbürsten}

Um alle derzeit auf dem Markt angebotenen Arten elektrischer Zahnbürsten zu untersuchen und miteinander zu vergleichen, wurden für diese Studie eine rotierend-oszillierende Zahnbürste, eine schallaktive Zahnbürste und eine elektrisch unterstützte Handzahnbürste als Testzahnbürsten ausgewählt. Obwohl die Beurteilung der Reinigungswirkung nach drei- bzw. viermonatiger Verwendung bei den elektrisch unterstützten Handzahnbürsten aufgrund ihrer limitierten Batterielaufzeit erschwert ist, sollte auch dieser Zahnbürstentyp in der Studie untersucht werden.

\subsubsection{Plaquerevelator}

Um die den Zahnoberflächen anhaftenden Beläge sichtbar zu machen, wurde, wie in zahlreichen anderen klinischen Untersuchungen (SHARMA et al. 1998, CHAVA 2000, SCHAFER et al. 2003), Erythrosin-Lösung verwendet. Bei der Anwendung des Revelators ist zu beachten, dass die Verteilung der Lösung und die Anfärbung der zu beurteilenden Flächen unter anderem von der Einwirkzeit abhängig sind. Die Aktivität der Wangenmuskulatur scheint hierbei ebenfalls eine Rolle zu spielen (SAXER und YANKELL 1997a). Auch die Menge des aufgetragenen Erythrosins unterliegt Schwankungen, wodurch die Intensität der Anfärbung und somit die Beurteilung der Plaquemenge beeinflusst werden können. Um diese Schwankungen so gering wie möglich zu halten, wurde in jeder Sitzung nach demselben Prinzip angefärbt: Die Untersucherin verwendete pro Kiefer stets ein mit je einem Tropfen Erythrosin 
getränktes Schaumstoff-Pellet. Anschließend wurde die Testperson gebeten, die überschüssige Revelatormenge durch einmaliges Spülen des Mundes mit Wasser zu beseitigen.

\subsubsection{Testzahnpasta}

Wie von SAXER und YANKELL (1997b) empfohlen, verwendeten alle Probanden während der Laufzeit der Studie dieselbe Zahnpasta, um eine Beeinflussung der Ergebnisse durch die Art der Zahnpasta auszuschließen. In der vorliegenden Untersuchung benutzten die Probanden Odol Med 3-Zahnpasta (GlaxoSmithKline).

\subsection{Methodik}

\subsubsection{PZR}

Um eine möglichst ähnliche Ausgangssituation zu schaffen, erhielten alle Studienteilnehmer nach der Einteilung in die entsprechenden Gruppen eine professionelle Zahnreinigung.

\subsubsection{Auswahl der Mundhygiene-Indizes}

Das Ziel dieser Untersuchung war die Überprüfung des Einflusses der Gebrauchsdauer dreier verschiedener elektrischer Zahnbürsten auf deren Reinigungseffektivität. Um die Mundhygiene der Probanden objektiv beurteilen und somit Rückschlüsse auf die Effizienz der verwendeten Zahnbürste ziehen zu können, war die Erhebung parodontaler Indizes obligat. Da ein Zusammenhang zwischen mikrobiellen Zahnbelägen und entzündlichen Veränderungen des Parodontiums besteht (LÖE et al. 1965, MÜLLER 2001, RATEITSCHAK et al. 2004), wurden für diese Studie zur Beurteilung der Mundhygiene ein Plaqueindex und zwei Gingivaindizes ausgewählt.

\subsubsection{Plaqueindex}

Die Plaquemenge auf den koronalen Zahnoberflächen wurde mit Hilfe des QHI in der von TURESKY et al. (1972) modifizierten Form ermittelt. Da dieser Index an sechs Messpunkten pro Zahn erhoben wird, eignet er sich besonders gut zur genaueren Bewertung der Approximal-bereiche. Laut Richtlinienkonzept der American Dental Association (ADA 1998) wird der nach Turesky modifizierte QHI als „verlässlich“ bis „sehr verlässlich“ eingestuft und daher für Zahnbürstenstudien empfohlen. Da an jedem zu bewertenden Zahn sechs Messungen durchzuführen sind, ist die Erhebung des QHI im Vergleich zu anderen Plaqueindizes relativ aufwändig, jedoch bietet der Index den Vorteil der Quantifizierung der Plaque. Bei der 
Erhebung des Approximalraum-Plaqueindex (API) nach LANGE hingegen erfolgt die Erfassung der Zahnbeläge lediglich nach dem Ja-Nein-Prinzip und mit einer anschließenden prozen-tualen Auswertung (LANGE et al. 1977); eine Aussage über die Plaquemenge wird nicht getroffen. Daher war die Erhebung des API für die Durchführung der vorliegenden Studie ungeeignet.

\subsubsection{Gingivaindizes}

Die alleinige Erhebung eines Plaqueindex lässt keine ausreichende Bewertung der Mundhygiene zu, da der Proband durch außergewöhnlich gründliche Zahnpflege unmittelbar vor dem jeweiligen Kontrolltermin die Testergebnisse verfälschen kann. Um eine derartige Verfälschung auszuschließen und um die Mundhygiene möglichst exakt zu bewerten, sollte ein Plaqueindex immer in Kombination mit mindestens einem Gingivaindex erhoben werden. Da sich eine Gingivitis bei inadäquater Plaqueentfernung stets verzögert etabliert und umgekehrt erst nach mehreren Tagen der Plaquefreiheit abklingt (LÖE et al. 1965), kann die Qualität der Mundhygiene nur durch die zusätzliche Erhebung eines Gingivaindex verlässlich klassifiziert werden.

Um den gingivalen Zustand besonders präzise beurteilen zu können, wurden in dieser Untersuchung sowohl ein invasiver (PBI), als auch ein non-invasiver Gingivaindex (GI) erhoben.

\subsection{Papillen-Blutungs-Index}

In der Zahnmedizin stehen verschiedene Indizes zur Verfügung, um den Grad der gingivalen Entzündung möglichst objektiv einstufen zu können. Als Beispiel sei neben dem PBI (SAXER und MÜHLEMANN 1975), welcher die Blutungsintensität beurteilt, der SulkusBlutungs-Index (SBI) genannt (MÜHLEMANN und SON 1971). Der wesentliche Nachteil des SBI ist, dass dieser Index lediglich mit einer Ja-/Nein-Entscheidung Auskunft über die Blutungsneigung der Gingiva gibt; die Intensität der Blutung kann durch den SBI nicht eingestuft werden. Daher wurde für die Durchführung der vorliegenden Arbeit der PBI verwendet, der den papillären Entzündungsgrad als Indikator für die Interdentalhygiene heranzieht. Aufgrund seiner Spezifität und Graduierung ist der PBI besonders für die individuelle Befunderhebung geeignet (RATEITSCHAK et al. 2004) und wird als „verlässlich“ eingestuft.

\subsection{Gingivaindex}

Obwohl bei der Erhebung des primär non-invasiven Gingivaindex (GI) nach LÖE und SILNESS (1963) auf das Kriterium „Blutung bei Sondierung“ verzichtet wird, verbleiben zur 
Beurteilung des Entzündungsgrades der Gingiva die Leitsymptome Farb- und Formveränderung. Diese sind zwar in den Anfangsstadien einer Gingivitis nur mäßig ausgeprägt und bei einer kurzen Testphase nicht adäquat zu beurteilen, bei einer Studiendauer von mehr als 24 Wochen kann der gingivale Zustand mit Hilfe des GI jedoch besonders präzise bewertet werden.

\subsection{Ergebnisse}

\subsubsection{Rotierend-oszillierende und schallaktive Zahnbürste (Gruppen A-D)}

Die Ergebnisse der vorliegenden Untersuchung zeigten, dass die Effektivität der Plaqueentfernung und somit der Grad der gingivalen Entzündung mit der Nutzungsdauer der Zahnbürsten korrelieren, unabhängig von den in der vorliegenden Arbeit untersuchten Zahnbürsten-typen. Dabei unterschieden sich die Parameter Plaquebefall (QHI) und gingivale Entzündung (PBI und GI) signifikant zwischen vierwöchigem und sechsmonatigem Gebrauch. So zeigte sich beim vierwöchigen Zahnbürstenwechsel eine in etwa konstante Verbesserung des Plaqueindex gegenüber dem Baseline-Wert über den gesamten Zeitraum bei beiden Zahnbürstentypen. Dieser Effekt ließ sich in der Gruppe der 6-Monats-Anwender nur in einem Zeitraum von vier Monaten feststellen. Zwischen Woche 16 und Woche 24 war schließlich ein erhöhter Plaquebefall, besonders in Gruppe A (rotierend-oszillierende Zahnbürste), zu beobachten: In der 6-Monats-Verschleißgruppe ergaben sich, verglichen mit der Baseline, höhere QHI-Werte bei Anwendung der rotierend-oszillierenden Zahnbürste als bei Verwendung der Schallzahnbürste. Dieses Phänomen könnte in den individuellen Unterschieden der Borstenbeschaffenheit begründet sein. Vorstellbar ist, dass die Borsten der rotierend-oszillierenden Zahnbürste schneller verschleißen bzw. unelastisch werden und somit keine gleichbleibend gute Plaquereduktion gewährleistet werden kann. Dass die Effektivität der Plaqueentfernung mit zunehmender Bürstenabnutzung sinkt, konnten GLAZE und WADE 1986 in ihrer klinischen Studie mit zwei Verschleißgruppen konstatieren. Während die eine Gruppe über einen Zeitraum von zehn Wochen dieselbe Bürste benutzte, erhielten die Probanden der anderen Gruppe während der gleichen Zeitspanne alle zwei Wochen eine neue Zahnbürste. Zwar stieg der Plaqueindex zwischen Woche zwei und Woche zehn in beiden Gruppen an, jedoch zeigte sich, dass Probanden, die während der gesamten Versuchsperiode mit derselben Zahnbürste putzten, signifikant mehr Plaque aufwiesen als Probanden, die regelmäßig mit neuen Bürsten versorgt wurden. Die Reinigungseffektivität der getesteten Zahnbürsten sank mit zunehmender Borstenermüdung (Vgl. Kapitel 2.8.1). 
Eine vergleichbare Tendenz zeigte auch der zeitliche Verlauf des PBI: Während die erhobenen Werte in der Gruppe des vierwöchigen Zahnbürstenwechsels unabhängig vom verwendeten Bürstentyp lediglich geringen Schwankungen unterlagen, verschlechterten sie sich bei denjenigen Probanden, die über sechs Monate mit einer rotierend-oszillierenden Zahnbürste putzten, signifikant gegenüber der Baseline. Wie bereits der zeitliche Verlauf des QHI erwarten ließ, stiegen auch die mit dem Biofilm korrelierenden Entzündungswerte in dieser Probandengruppe deutlich an, wohingegen sie bei Verwendung der Schallzahnbürste auch noch nach einer Nutzungsdauer von sechs Monaten konstant blieben. Da ein Zusammenhang zwischen mikrobiellen Zahnbelägen und entzündlichen Veränderungen des Parodontiums besteht (LÖE et al. 1965, MÜLLER 2001, RATEITSCHAK et al. 2004), überrascht der beschriebene Effekt nicht. Im Gegensatz hierzu konnte in der Gruppe der 6-Monats-Anwender der schallaktiven Zahnbürste sogar noch nach Woche 24 eine Verbesserung des PBI im Vergleich zur Baseline beobachtet werden. Dieser Effekt hängt möglicherweise damit zusammen, dass die Probanden der Gruppe A (rotierende Zahnbürste) einen ohnehin deutlich geringeren BaselinePBI aufwiesen $(0.12 \pm 0.15)$ als Testpersonen der Gruppe C (Schallzahnbürste) mit einem Baseline-PBI von (0.32 \pm 0.21$)$, sodass eine Verbesserung des Index in Gruppe A kaum realistisch erschien. An dieser Stelle sei noch einmal erwähnt, dass die Randomisierung gemäß Plaqueindex (QHI) und nicht gemäß PBI erfolgt war. Verglichen mit dem am Tag 14 erhobenen PBI $(0.20 \pm 0.16)$ zeigte sich allerdings auch bei Verwendung der Schallzahnbürste ein höherer Wert nach Ablauf der sechs Monate (0,28 \pm 0.18$)$.

Korrespondierend mit den Ergebnissen des PBI zeigte die Auswertung des GI, dass sich der zeitliche Verlauf in der Gruppe der 4-Wochen-Anwender signifikant vom Verlauf in der Gruppe des sechsmonatigen Gebrauchs unterschied: Unabhängig vom Zahnbürstentyp verschlechterte sich der GI bei den 6-Monats-Anwendern in Woche 24 im Vergleich zur Baseline. Dagegen blieben die Werte beim vierwöchigen Zahnbürstenwechsel über den gesamten Zeitraum stabil. Bei Anwendern der rotierenden Bürste ergab sich nach Ablauf der 24 Wochen ein, verglichen mit Baseline, signifikant schlechterer GI ( $\mathrm{p}=0.0341)$. Auch bei Probanden, die über 24 Wochen die Schallzahnbürste anwendeten, verschlechterte sich der GI, jedoch waren hier die Veränderungen nicht signifikant.

In der wissenschaftlichen Literatur wird der Einfluss der Zahnbürstenabnutzung auf deren Reinigungswirkung kontrovers diskutiert. Klinische Studien mit künstlich abgenutzten Borsten stellten eine weniger effektive Plaqueentfernung im Vergleich zu neuen Zahnbürsten heraus (WARREN PR et al. 2002, KOCH et al. 2007). Auch andere Autoren erkannten, dass abgenutzte Zahnbürsten eine deutlich geringere Reinigungseffektivität besitzen als weniger 
abgenutzte. Der Abnutzungsstatus der untersuchten Zahnbürsten beeinflusste die Reinigungsfähigkeit, wobei die Plaquereduktion umso unzureichender wurde, je schwieriger die zu reinigenden Regionen zu erreichen waren. Nach Ansicht der Autoren könnten sowohl die Borstenermüdung als auch das mit zunehmender Gebrauchsdauer auftretende Verkürzen der Borsten zu einer solchen Verschlechterung führen (KREIFELDT et al. 1980) (Vgl. Kapitel 2.8.1.)

Verschiedene klinische Untersuchungen mit „natürlich abgenutzten“ Borsten zeigen keine verminderte Effizienz hinsichtlich der Plaqueentfernung im Vergleich zu neuen Zahnbürsten (SFORZA et al. 2000, CONFORTI et al. 2003). In den meisten Studien ist der Untersuchungszeitraum jedoch auf maximal vier Monate begrenzt (DALY et al. 1996, TAN und DALY 2002, HEDGE et al. 2005), sodass sich hinsichtlich der Plaqueentfernung keine nennenswerten Unterschiede ergaben. In der vorliegenden Arbeit umfasste der Untersuchungszeitraum jedoch sechs Monate. So konnte gezeigt werden, dass die Reinigungswirkung der getesteten Zahnbürsten erst nach einem Beobachtungszeitraum von etwa vier Monaten nachlässt, in den ersten 16 Wochen hingegen konnte eine gleichbleibend gute Plaqueentfernung erzielt werden. So ist die vorliegende Studie die erste, welche diesen Sachverhalt im Rahmen einer klinischen Untersuchung aufzeigt.

Über den Einfluss der Nutzungsdauer von Zahnbürsten auf den gingivalen Zustand existieren nur wenige Untersuchungen. GLAZE und WADE (1986) konnten bei der gingivalen Beurteilung keine statistisch signifikanten Unterschiede zwischen Probanden, die über zehn Wochen mit derselben Zahnbürste putzten, und Probanden, die alle zwei Wochen eine neue Zahnbürste verwendeten, feststellen (Vgl. Kapitel 2.8.3). Demgegenüber zeigen die Ergebnisse der vorliegenden Untersuchung, dass die gingivalen Verhältnisse (PBI) bis zu einer Gebrauchsdauer von vier Monaten stabil sind, vom fünften Monat an ist jedoch mit vermehrten Entzündungszeichen der Gingiva (PBI und GI) zu rechnen.

Ein durch das Putzen mit abgenutzten Borsten hervorgerufenes Trauma wird in keiner der bisher zitierten Studien erwähnt, dieser Aspekt wurde in der vorliegenden Untersuchung ebenfalls nicht erfasst. Vielmehr gehen einige Autoren davon aus, dass abgenutzte Borsten tendenziell weicher und daher zwar hinsichtlich der Plaquereduktion ineffektiver werden (KREIFELDT et al. 1980), dass deren Verletzungspotenzial allerdings im Vergleich zu neuen, unbenutzten Borsten geringer ausfalle.

Ein weiterer Effekt, der nicht nur bei der Entwicklung aller Hygieneindizes, sondern auch in fast allen Gruppen beobachtet werden konnte, war eine verbesserte Mundhygiene an Tag 14 im Vergleich zu Tag 0 (Baseline). Zurückzuführen ist dies vermutlich auf die am Tag 0 durchgeführte Zahnreinigung, durch die bei allen Probanden eine nahezu vollständige 
Plaquefreiheit erreicht wurde, welche nach 14 Tagen in einer Verringerung der gingivalen Entzündungszeichen resultieren könnte. Als weitere vorstellbare Ursache für den oben beschriebenen Effekt ist ein möglicher „Motivationsschub“ der Probanden zu Studienbeginn, bedingt durch die neue elektrische Zahnbürste, zu diskutieren. So könnte besonders gründliches Putzen zu Beginn der Studie zu einer Verminderung der erhobenen Indizes geführt haben. Das im Vergleich zur Baseline-Untersuchung insgesamt bessere Mundhygieneniveau, welches sich bei allen Indizes sowie in beiden Verschleißgruppen bis zum Ablauf der ersten vier Monate zeigte, könnte ebenfalls durch den oben genannten „Motivationsschub“ begründet sein, sofern dieser auch über die Phase des Studienbeginns angehalten haben sollte. Denkbar wäre allerdings auch, dass die Studienteilnehmer jeweils unmittelbar vor den Kontrollterminen besonders intensiv putzen, um dem Untersucher möglichst einwandfreie oralhygienische Verhältnisse zu präsentieren. Zumindest beim Parameter Plaquebefall wäre eine derartige Verfälschung des Testergebnisses vorstellbar. Um diesen Effekt zu vermeiden, erfolgte die Erhebung des Plaqueindex in Kombination mit zwei Entzündungsindizes (Vgl. Kapitel 5.3.3.2).

Eine Verbesserung der Mundhygiene-Indizes nach dem einwöchigen Gebrauch einer neuen Zahnbürste (zwischen Woche 24 und 25) konnte vor allem bei denjenigen Probanden beobachtet werden, welche über die gesamten 24 Wochen zuvor dieselbe Zahnbürste benutzt hatten. So lagen die Mittelwerte aller Indizes bei den 6-Monats-Anwendern in Woche 25 stets unter den Mittelwerten der in der Vorwoche erhobenen Indizes. Folglich scheint eine neue, nicht abgenutzte Bürste in der Lage zu sein, den Biofilm effektiver zu beseitigen und somit entzündlichen Veränderungen des Parodontiums vorzubeugen als eine Zahnbürste, die über einen Zeitraum von sechs Monaten verwendet wurde. Als Ursachen hierfür sind wiederum Verschleißerscheinungen wie Borstenermüdung, Aufspreizung der Borsten und mangelnde Borstenelastizität zu nennen (Vgl. Kapitel 2.8.1).

\subsubsection{Elektrisch unterstützte Handzahnbürste (Gruppen E und F)}

Da aufgrund der limitierten Batterielaufzeit der elektrisch unterstützten Handzahnbürsten eine Studiendauer von sechs Monaten in den Gruppen E und F nicht möglich war, ist ein unmittelbarer Vergleich mit den beiden anderen Testzahnbürsten nicht statthaft. Die Diskussion der Ergebnisse der untersuchten Handzahnbürsten erfolgt daher separat.

Sowohl beim Plaqueindex, als auch bei den beiden Gingivaindizes zeigten sich die höchsten Mittelwerte jeweils an jenem Kontrolltermin, der unmittelbar nach dem Ende der Batterie- 
laufzeit der getesteten Zahnbürsten, also nach der maximalen Gebrauchsdauer, stattfand. Ähnlich wie bei den beiden anderen untersuchten Bürsten würde das Auftreten dieses Effekts dafür sprechen, dass die Reinigungswirkung einer Zahnbürste mit zunehmender Verwendungsdauer nachlässt. Mit Ausnahme des Plaqueindex in Gruppe E verbesserten sich die Mittelwerte aller drei Mundhygieneindizes im Zeitraum zwischen der Eingangsuntersuchung (Tag 0) und Tag 14. Wie bereits diskutiert, ist als Ursache hierfür die an Tag 0 durchgeführte Zahnreinigung oder aber eine zu Studienstart auftretende, besonders hohe Motivation der Testpersonen anzunehmen. Des Weiteren stellten sich die während der Abschlusskontrolle ermittelten Werte aller erhobenen Indizes in beiden Gruppen niedriger dar als die Werte, die beim letzten Kontrolltermin (Ausgabe einer neuen Zahnbürste) erhoben wurden. Die Ausnahme bildete hier allerdings der GI in der Gruppe der 4-Wochen-Anwender. Die Verbesserung der Mundhygiene nach einwöchigem Putzen mit einer neuen Zahnbürste gibt, wie bereits diskutiert, Grund zur Annahme, dass eine neue, unbenutzte Zahnbürste eine bessere Reinigungswirkung besitzt als eine bereits benutzte Zahnbürste. Die effektivere Plaquereduktion würde somit auch den Rückgang der Entzündungsparameter (GI und PBI) erklären, da sich eine Gingivitis bei inadäquater Plaqueentfernung stets verzögert etabliert und umgekehrt erst nach mehreren Tagen der Plaquefreiheit abklingt (LÖE et al. 1965).

Die relativ stark variierenden Akku-Laufzeiten (Mittelwert $=84.45 \pm 9.24$ Tage) in der Gruppe, die über den gesamten Zeitraum mit derselben Zahnbürste putzte, sind vermutlich weniger durch individuelle Unterschiede der Geräte vom Werk zu begründen, sondern vielmehr als Resultat unterschiedlichen Zahnputzverhaltens bzw. differierender Zahnputzzeiten zu werten. 


\section{Zusammenfassung}

Das wichtigste Hilfsmittel oralhygienischer Maßnahmen ist die Zahnbürste. Da diese als täglich verwendeter Gebrauchsgegenstand gewissen Verschleißvorgängen unterliegt, sollte ein regelmäßiger Austausch erfolgen (KOCH et al. 2007). Während im Allgemeinen ein Zahnbürstenwechsel nach etwa 12 Wochen angeraten wird, besteht in der Literatur hinsichtlich dieser Empfehlung und der Korrelation von Zahnbürstenabnutzung und -effektivität Uneinigkeit; wissenschaftliche Grundlagen für einen Nutzungszeitraum von drei Monaten finden sich kaum.

Gegenstand der vorliegenden Studie war die Untersuchung des Einflusses der Anwendungsdauer von verschiedenen elektrischen Zahnbürsten auf die Plaquereduktion und den gingivalen Zustand. Hierbei sollte die Hypothese, dass die Reinigungswirkung einer Zahnbürste mit zunehmender Gebrauchsdauer nachlässt, überprüft werden.

Um festzustellen, inwiefern die Reinigungseffizienz einer Zahnbürste mit ihrer Gebrauchsdauer korreliert, wurden drei unterschiedliche Typen elektrischer Zahnbürsten an 71 gesunde Testpersonen im Alter zwischen 20 und 70 Jahren ausgegeben. Die Studie folgte einem untersucherblinden Parallel-Design. Die Testzahnbürsten wurden auf drei Gruppen, randomisiert gemäß Baseline-QHI, verteilt. Nach der Screening-Untersuchung erhielten alle Probanden eine professionelle Zahnreinigung. Jede der drei „Zahnbürstengruppen“ wurde in zwei Untergruppen eingeteilt, von denen jeweils einer Gruppe alle vier Wochen eine neue Bürste zugewiesen wurde (Verschleißgruppe 1). Die Probanden der zweiten Untergruppe putzten über sechs Monate (bzw. bis zum Ende der Batterielaufzeit in den Gruppen E und F) mit derselben Bürste (Verschleißgruppe 2). In den während des Studienverlaufs stattfindenden Kontrollterminen nach zwei, acht, zwölf, 16, 24 und 25 Wochen wurden jeweils ein Plaqueindex (QHI) sowie zwei Gingivaindizes (PBI und GI) erhoben, um den Mundhygienezustand der Probanden zu bewerten und somit Rückschlüsse auf die Reinigungswirkung der Zahnbürste zu ziehen. Während der gesamten Testphase verwendeten die Probanden die jeweilige Testzahnbürste mit einheitlicher Zahnpasta zweimal täglich für zwei Minuten.

Die gewonnenen Daten wurden zur statistischen Auswertung folgender Zusammenhänge verwertet:

- Vergleich des zeitlichen Verlaufs der Indizes in den verschiedenen Verschleißgruppen

- Vergleich der Bürstentypen untereinander.

Die Auswertung ergab bei den Parametern Plaquebefall (QHI) und gingivale Entzündung (PBI, GI) bei den getesteten Zahnbürsten in der Gruppe der 6-Monats-Anwender signifikant 
schlechtere Werte als bei Testpersonen, die alle vier Wochen eine neue Zahnbürste verwendeten. Somit wurde konstatiert, dass die Effektivität der Plaqueentfernung und infolgedessen der Grad der gingivalen Entzündung mit der Nutzungsdauer einer Zahnbürste korrelieren, unabhängig von den in der vorliegenden Arbeit untersuchten Zahnbürstentypen.

Allerdings ließ die Auswertung der Plaque- und Entzündungsindizes vermuten, dass die Verschleißerscheinungen bei der rotierend-oszillierenden Zahnbürste schneller auftreten als bei der schallaktiven Bürste. Zwischen Woche 16 und Woche 24 war ein erhöhter Plaquebefall bei den Anwendern der rotierend-oszillierenden Zahnbürste zu beobachten. Ähnliches zeigte sich auch im zeitlichen Verlauf des PBI: Bei Probanden, die über sechs Monate mit einer rotierend-oszillierenden Zahnbürste putzten, stiegen die Werte deutlich gegenüber der Baseline an, wohingegen sie bei Verwendung der Schallzahnbürste auch nach einer Nutzungsdauer von sechs Monaten konstant blieben. Die Auswertung des GI ergab ebenfalls signifikant höhere Werte bei Anwendern der rotierend-oszillierenden Bürste nach Ablauf der 24 Wochen, während sich bei Verwendern der Schallzahnbürste nach sechs Monaten keine signifikante Verschlechterung des Index zeigte. Die erhobenen Werte bei den 4-Wochen-Anwendern unterlagen, unabhängig vom verwendeten Bürstentyp, lediglich geringen Schwankungen.

Anwender der elektrisch unterstützten Handzahnbürsten (Gruppe E und F) wiesen die höchsten Plaque- und Entzündungsindizes jeweils an demjenigen Kontrolltermin auf, der unmittelbar nach dem Ende der Batterielaufzeit der getesteten Zahnbürsten stattfand, also nach der maximalen Anwendungsdauer.

Die Ergebnisse der vorliegenden Untersuchung deuten an, dass die Reinigungswirkung einer Zahnbürste mit zunehmender Nutzungsdauer abnimmt. Während das Ausmaß des Plaquebefalls und die gingivalen Verhältnisse bis zu einer Gebrauchsdauer von etwa vier Monaten stabil waren, waren ab Beginn des fünften Monats erhöhte Plaquemengen (QHI) und vermehrte Entzündungszeichen der Gingiva (PBI und GI) zu verzeichnen.

Die Tatsache, dass die Abnutzung der Bürsten durch verschiedene individuelle Faktoren beeinflusst wird, erschwert die Angabe einer allgemeingültigen Gebrauchsdauer einer Zahnbürste. Vor allem über die Abnutzung und das Austauschintervall elektrischer Zahnbürsten existieren kaum wissenschaftliche Grundlagen, jedoch sollte nach Herstellerangaben ein regelmäßiger Austausch nach drei Monaten stattfinden.

Die Ergebnisse dieser Untersuchung legen einen Anwendungszeitraum von zwölf bis maximal 16 Wochen nahe, da sich die Mundhygiene-Indizes anschließend bei allen Probanden verschlechterten. Folglich ist nach einem Nutzungsintervall von mehr als vier Monaten mit einer reduzierten Reinigungswirkung zu rechnen. 


\section{Anhang}

\subsection{Anamnesebogen}

\section{Fragebogen}

„Klinische Studie zur Überprüfung des Einflusses der Gebrauchsdauer verschiedener elektrischer Zahnbürsten auf die mechanische Plaquekontrolle und den gingivalen Zustand“

Name:

Adresse:

Geburtsdatum:

Telefon:

Pat.-Nr.:

Bitte beantworten Sie die folgenden Fragen bzw. kreuzen Sie j a oder n e i n an.

Die gewissenhafte Beantwortung ist eine Voraussetzung für eine erfolgreiche Behandlung!

1. Sind Sie in ständiger ärztlicher Behandlung?

ja nein

Wenn ja, weswegen?

2. Sind Sie Diabetiker? Insulinpflichtig? (HbA1c Wert?

3. Leiden Sie an einer Herzerkrankung? (z.B. A. pectoris, Endokarditis, Klappenfehler)

4. Leiden Sie an Bluthochdruck (Werte?

5. Müssen Sie ständig Medikamente einnehmen?

Wenn ja, welche?

(z.B. zur Blutzuckersenkung; gegen Herzbeschwerden, Bluthochdruck; zur

Hemmung der Blutgerinnung; Rheumamittel; Beruhigungs-/Schlaftabletten)

6. Sind Sie zur Zeit in ärztlicher Behandlung?

Wenn ja, weswegen?

7. Nehmen Sie zur Zeit Medikamente ein?

Wenn ja, welche?

8. Haben Sie in den letzten 3 Monaten ein Antibiotikum einnehmen müssen? Wenn ja, weshalb?

9. Nehmen Sie Hormonpräparate ein?

Wenn ja, welche?

10. Sind Sie je operiert worden?

11. Sind Ihnen jemals Blut oder Blutprodukte übertragen worden?

Wenn ja, weswegen?

Wenn ja, welche?

(z.B. Schmerzmittel, Penicillin, Sulfonamide, Jod, Latex)

13. Haben Sie einen Allergie-Pass?

Wenn ja, für welche Substanzen?

14. Wann sind Sie zum letzten Mal zahnärztlich untersucht worden? 
15. Sind Ihre Zähne temperaturempfindlich?

$\left[\begin{array}{ll}{[} & {[}\end{array}\right]$

16. Blutet Ihr Zahnfleisch?

17. Bemerken Sie Stellungsveränderungen Ihrer Zähne?

18. Atmen Sie häufig durch den Mund?

19. Haben Sie manchmal einen schlechten Geschmack im Mund?

20. Haben Sie eine Zahnspange getragen?

21. Haben Sie wegen Zahnlockerung bzw. Zahnfleischbeschwerden schon einmal einen Zahnarzt aufgesucht?

Wenn ja, was wurde gemacht?

22. Haben Sie schon einmal einen Zahn verloren?

Wenn ja, was war die Ursache?

Karies ( ) Parodontose/ Zahnlockerung ( ) Trauma/Unfall

23. Wurde bei Ihnen bereits eine "Parodontose"-Behandlung durchgeführt? Wenn ja, wann?

24. Rauchen Sie oder haben Sie geraucht?

25. Wie viel Zigaretten/ Schachteln pro Tag etwa? . Zigaretten/ Tag, Schachteln/ Tag

26. In welchem Lebensalter haben Sie mit dem Rauchen begonnen? Mit Jahren

27. Vor wie viel Monaten/Jahren haben Sie mit dem Rauchen aufgehört? Vor Monaten/ Vor Jahren

28. Konsumieren Sie alkoholische Getränke? (Bier, Wein, Sekt, Spirituosen) nie [ ] gelegentlich [ ] täglich [ ]

29. Konsumieren Sie zuckerhaltige Genussmittel (z.B. gesüßte Getränke, Schokoriegel, Eis u. ä.)

nie [ ] gelegentlich [ ] täglich [ ] mehrmals täglich [ ]

30. Wie sehen Ihre Zahnputzgewohnheiten aus?
Wie oft putzen Sie? 1mal täglich
( ) morgens und abends nach jeder Mahlzeit ( ) seltener

Wie putzen Sie? Handzahnbürste ( ) elektrische Zahnbürste ( )

$\begin{array}{llll}\text { Benutzen Sie Zahnseide? } & \text { Nie ( ) wöchentlich ( ) } \\ & \text { Gelegentlich ( ) täglich ( ) }\end{array}$

Leiden/ Litten Sie an folgenden Erkrankungen? (Bitte ankreuzen)

[ ] Haben Sie häufig Erkältungskrankheiten?

[ ] Sind Sie ständig durstig?

[ ] Bekommen Sie schnell blaue Flecken?

[ ] Leiden Sie an längeren Blutungen, z.B. nach Schnittverletzungen oder Zahnextraktion?

Sonstige Angaben:

Göttingen, den Unterschrift: 


\subsection{Patientenaufklärung}

Prof. Dr. med. dent. R. Mausberg; Georg-August-Universität Göttingen, Zentrum Zahn-, Mund- und Kieferheilkunde; Abteilung Präventive Zahnheilkunde, Kariologie und Parodontologie; Robert-Koch-Str. 40, 37075 Göttingen; Tel. 0551/39 22878

\section{Patientenaufklärung}

„Klinische Studie zur Überprüfung des Einflusses der Gebrauchsdauer verschiedener elektrischer Zahnbürsten auf die mechanische Plaquekontrolle und den gingivalen Zustand“

\section{SEHR GEEHRTER PATIENT!}

Dentale Plaque (Zahnbelag) ist Ursache von Karies und entzündlichen Veränderungen an Zahnfleisch bzw. Zahnhalteapparat. Plaque ist ein sogenannter Biofilm, der sich ausschließlich mechanisch, d. h. durch Verwendung von Zahnbürste, Zahnpasta und Zahnseide entfernen lässt. Plaquekontrolle ist der effektivste Weg zur Vermeidung von Karies und Zahnfleischentzündungen, und ist daher nicht nur Voraussetzung für die Schaffung gesunder oraler Verhältnisse, sondern auch unabdingbar für den dauerhaften Erhalt der Zahngesundheit.

Diese wissenschaftliche Untersuchung soll Aufschluss darüber geben, ob Alter und Abnutzung von elektrischen Zahnbürsten die Plaquekontrolle und das Zahnfleischverhalten beeinflussen.

Um Aussagen über den jeweiligen Mundhygienezustand treffen zu können, werden spezielle Indizes erhoben, welche zum einen die Menge der Plaque erfassen (Plaqueindex), zum anderen der Beurteilung des Entzündungsgrades des Zahnfleisches dienen (Entzündungsindex). Mit Hilfe dieser Indizes können somit Rückschlüsse auf die Reinigungswirkung bzw. Effektivität der verwendeten Zahnbürste gezogen werden.

Die Untersuchung Ihrer Zähne bzw. Ihres Zahnfleischs führt eine Doktorandin (Zahnärztin) unserer Abteilung durch. Des Weiteren bitten wir Sie, einige Fragen zu Ihrem Allgemeinzustand, Ihren Lebensgewohnheiten und Zahnpflegegewohnheiten zu beantworten.

Risiken und Nebenwirkungen bei der Durchführung der Studie sind nicht zu erwarten, da keine Medikamente oder Röntgenaufnahmen notwendig sind. Ihre Patientendaten und Angaben werden pseudonymisiert, nur zu Forschungszwecken verwendet und nach Auswertung ver- 
nichtet. Der Zeitraum für die Untersuchung ist ein halbes Jahr. Während dieses Untersuchungszeitraumes sollten keine weiteren Mundhygienehilfsmittel Anwendung finden. Die Terminvergabe für die Kontrolluntersuchungen erfolgt nach individueller Absprache.

Wir bitten um die freiwillige Teilnahme an der Studie. Sie können die Teilnahme jederzeit widerrufen, ohne Angabe von Gründen und ohne Nachteile erwarten zu müssen. 


\subsection{Einverständniserklärung}

Prof. Dr. med. dent. R. Mausberg; Georg-August-Universität Göttingen, Zentrum Zahn-, Mund- und Kieferheilkunde; Abteilung Präventive Zahnheilkunde, Kariologie und Parodontologie; Robert-Koch-Str. 40, 37075 Göttingen; Tel. 0551/3922878

\section{Einwilligungserklärung}

„Klinischen Studie zur Überprüfung des Einflusses der Gebrauchsdauer verschiedener elektrischer Zahnbürsten auf die mechanische Plaquekontrolle und den gingivalen Zustand“

Ich, wurde von meinem Arzt vollständig über Wesen, Bedeutung und Tragweite der klinischen Prüfung mit dem Titel „Klinischen Studie zur Überprüfung des Einflusses der Gebrauchsdauer verschiedener elektrischer Zahnbürsten auf die mechanische Plaquekontrolle und den gingivalen Zustand“ aufgeklärt.

Ich habe den Aufklärungstext gelesen und verstanden. Ich hatte die Möglichkeit, Fragen zu stellen, habe die Antworten verstanden und akzeptiere diese. Mein Arzt hat mich über die mit der Teilnahme an der Studie verbundenen Risiken und den möglichen Nutzen informiert.

Mir ist bekannt, dass bei dieser Untersuchung personenbezogene Daten, insbesondere medizinische Befunde, über mich erhoben, gespeichert und ausgewertet werden sollen. Die Verwendung der Angaben über meine Gesundheit erfolgt nach gesetzlichen Bestimmungen und setzt vor der Teilnahme an der klinischen Prüfung die folgende freiwillig abgegebene Einwilligungserklärung voraus.

Ich hatte ausreichend Zeit, mich zur Teilnahme an dieser Untersuchung zu entscheiden und weiß, dass die Teilnahme freiwillig ist.

Mir ist bekannt, dass ich jederzeit und ohne Angaben von Gründen diese Zustimmung widerrufen kann, ohne dass sich dieser Entschluss nachteilig auswirkt.

Ich habe eine Kopie der Patienteninformation und dieser Einwilligungserklärung erhalten. Ich erkläre hiermit meine freiwillige Teilnahme an dieser Studie. 
7.4 Dokumentationsbögen zur Erhebung der Indizes

Woche:

Datum:

QHI modifiziert nach Turesky

\begin{tabular}{|c|c|c|c|c|c|c|c|c|c|c|c|c|c|c|c|c|c|}
\hline & 18 & 17 & 16 & 15 & 14 & 13 & 12 & 11 & 21 & 22 & 23 & 24 & 25 & 26 & 27 & 28 & \\
\hline Bukkal & & & & & & & & & & & & & & & & & Bukkal \\
\hline Oral & & & & & & & & & & & & & & & & & Oral \\
\hline Oral & & & & & & & & & & & & & & & & & Oral \\
\hline Bukkal & & & & & & & & & & & & & & & & & Bukkal \\
\hline & 48 & 47 & 46 & 45 & 44 & 43 & 42 & 41 & 31 & 32 & 33 & 34 & 35 & 36 & 37 & 38 & \\
\hline
\end{tabular}

Wert:

PBI

\begin{tabular}{|c|c|c|c|c|c|c|c|c|c|c|c|c|c|c|c|c|c|}
\hline & 18 & 17 & 16 & 15 & 14 & 13 & 12 & 11 & 21 & 22 & 23 & 24 & 25 & 26 & 27 & 28 & \\
\hline Bukkal & & & & & & & & & & & & & & & & & Oral \\
\hline Oral & & & & & & & & & & & & & & & & & Bukkal \\
\hline & 48 & 47 & 46 & 45 & 44 & 43 & 42 & 41 & 31 & 32 & 33 & 34 & 35 & 36 & 37 & 38 & \\
\hline
\end{tabular}

Wert:

GI nach Löe und Silness

\begin{tabular}{|c|c|c|c|c|c|c|c|c|c|c|c|c|c|c|c|c|c|}
\hline & 18 & 17 & 16 & 15 & 14 & 13 & 12 & 11 & 21 & 22 & 23 & 24 & 25 & 26 & 27 & 28 & \\
\hline Bukkal & & & & & & & & & & & & & & & & & Bukkal \\
\hline Oral & & & & & & & & & & & & & & & & & Oral \\
\hline Oral & & & & & & & & & & & & & & & & & Oral \\
\hline Bukkal & & & & & & & & & & & & & & & & & Bukkal \\
\hline & 48 & 47 & 46 & 45 & 44 & 43 & 42 & 41 & 31 & 32 & 33 & 34 & 35 & 36 & 37 & 38 & \\
\hline
\end{tabular}

Wert: 
Name des Patienten:

Gruppe:

\begin{tabular}{|c|l|l|l|l|l|}
\hline & Datum & QHI & PBI & GI & DMF-T \\
\hline Tag 0 & & & & \\
\hline Tag 14 & & & & \\
\hline Woche 8 & & & & \\
\hline Woche 12 & & & & \\
\hline Woche 16 & & & & \\
\hline Woche 24 & & & & \\
\hline Woche 25 & & & & \\
\hline
\end{tabular}




\subsection{Excel-Tabelle zur statistischen Auswertung der Indizes}

\section{Legende:}

Pat.: $\quad$ Patient (laufende Nummer)

ZB: $\quad$ in der Studie verwendete Zahnbürste

Verschl.: Verschleißgruppe: $0=4$ Wochen, $1=6$ Monate

ZB vorher: Zahnbürste, die der Proband gewohnheitsgemäß vor der Studienteilnahme benutzte: 0 = Handzahnbürste, 1 = elektrische Zahnbürste,

2 = sowohl Handzahnbürste, als auch elektrische Zahnbürste

R/NR: $\quad$ Raucher/Nichtraucher: $0=$ Raucher, $1=$ Nichtraucher

Geschl.: Geschlecht des Probanden: $0=\hat{\jmath}, 1=q$

Alter: $\quad$ Alter des Probanden

Laufz. in d: Laufzeit der Batterie der elektrisch unterstützten Handzahnbürste in Tagen (Gruppen E und F)

Akku leer: Kontrolltermin unmittelbar nach Ende der Akkulaufzeit

(Gruppen E und F)

n.b.: nicht bewertet

\begin{tabular}{|c|c|c|c|c|c|c|c|c|c|c|c|}
\hline Pat. & Zeitpunkt & ZB & Verschl. & ZB vorher & $\mathrm{R} / \mathrm{NR}$ & Geschl. & Alter & $\begin{array}{c}\text { DMF- } \\
\mathrm{T}\end{array}$ & $\mathrm{QHI}$ & $\mathrm{PBI}$ & $\mathrm{Gl}$ \\
\hline 1 & base & rotierend & 1 & 1 & 0 & 1 & 23 & 9 & 0,44 & n.b. & n.b. \\
\hline 1 & Tag 0 & rotierend & 1 & 1 & 0 & 1 & 23 & 9 & n.b. & 0,05 & 0,375 \\
\hline 1 & Tag 14 & rotierend & 1 & 1 & 0 & 1 & 23 & 9 & 0,792 & 0,05 & 0,302 \\
\hline 1 & Woche 8 & rotierend & 1 & 1 & 0 & 1 & 23 & 9 & 0,7 & 0,05 & 0,333 \\
\hline 1 & Woche 12 & rotierend & 1 & 1 & 0 & 1 & 23 & 9 & 0,485 & 0,05 & 0,396 \\
\hline 1 & Woche 16 & rotierend & 1 & 1 & 0 & 1 & 23 & 9 & 0,886 & 0,1 & 0,427 \\
\hline 1 & Woche 24 & rotierend & 1 & 1 & 0 & 1 & 23 & 9 & 1,045 & 0,2 & 0,552 \\
\hline 1 & Woche 25 & rotierend & 1 & 1 & 0 & 1 & 23 & 9 & 0,455 & 0 & 0,208 \\
\hline 2 & base & rotierend & 1 & 2 & 0 & 0 & 21 & 0 & 0,43 & n.b. & n.b. \\
\hline 2 & Tag 0 & rotierend & 1 & 2 & 0 & 0 & 21 & 0 & n.b. & 0,036 & 0,232 \\
\hline 2 & Tag 14 & rotierend & 1 & 2 & 0 & 0 & 21 & 0 & 0,607 & 0 & 0,018 \\
\hline 2 & Woche 8 & rotierend & 1 & 2 & 0 & 0 & 21 & 0 & 1,232 & 0,321 & 0,777 \\
\hline 2 & Woche 12 & rotierend & 1 & 2 & 0 & 0 & 21 & 0 & 1,304 & 0,143 & 0,964 \\
\hline 2 & Woche 16 & rotierend & 1 & 2 & 0 & 0 & 21 & 0 & 1,339 & 0,286 & 0,902 \\
\hline 2 & Woche 24 & rotierend & 1 & 2 & 0 & 0 & 21 & 0 & 1,393 & 0,429 & 1,152 \\
\hline 2 & Woche 25 & rotierend & 1 & 2 & 0 & 0 & 21 & 0 & 0,786 & 0 & 0,607 \\
\hline 3 & base & rotierend & 1 & 1 & 0 & 1 & 23 & 0 & 0,98 & n.b. & n.b. \\
\hline 3 & Tag 0 & rotierend & 1 & 1 & 0 & 1 & 23 & 0 & n.b. & 0 & 0,344 \\
\hline 3 & Tag 14 & rotierend & 1 & 1 & 0 & 1 & 23 & 0 & 0,326 & 0,15 & 0,229 \\
\hline 3 & Woche 8 & rotierend & 1 & 1 & 0 & 1 & 23 & 0 & 0,521 & 0,05 & 0,229 \\
\hline 3 & Woche 12 & rotierend & 1 & 1 & 0 & 1 & 23 & 0 & 0,451 & 0,1 & 0,458 \\
\hline 3 & Woche 16 & rotierend & 1 & 1 & 0 & 1 & 23 & 0 & 0,375 & 0,05 & 0,396 \\
\hline 3 & Woche 24 & rotierend & 1 & 1 & 0 & 1 & 23 & 0 & 0,507 & 0,15 & 0,563 \\
\hline 3 & Woche 25 & rotierend & 1 & 1 & 0 & 1 & 23 & 0 & 0,326 & 0 & 0,208 \\
\hline 4 & base & rotierend & 1 & 1 & 0 & 1 & 23 & 10 & 1,27 & n.b. & n.b. \\
\hline 4 & Tag 0 & rotierend & 1 & 1 & 0 & 1 & 23 & 10 & n.b. & 0,045 & 0,663 \\
\hline 4 & Tag 14 & rotierend & 1 & 1 & 0 & 1 & 23 & 10 & 0,963 & 0,455 & 0,5 \\
\hline 4 & Woche 8 & rotierend & 1 & 1 & 0 & 1 & 23 & 10 & 0,885 & 0,318 & 0,462 \\
\hline 4 & Woche 12 & rotierend & 1 & 1 & 0 & 1 & 23 & 10 & 1,391 & 0,227 & 0,519 \\
\hline 4 & Woche 16 & rotierend & 1 & 1 & 0 & 1 & 23 & 10 & 1,583 & 0,273 & 0,74 \\
\hline
\end{tabular}




\begin{tabular}{|c|c|c|c|c|c|c|c|c|c|c|c|}
\hline 4 & Woche 24 & rotierend & 1 & 1 & 0 & 1 & 23 & 10 & 1,692 & 0,591 & 1,038 \\
\hline 4 & Woche 25 & rotierend & 1 & 1 & 0 & 1 & 23 & 10 & 0,974 & 0,364 & 0,577 \\
\hline 5 & base & rotierend & 1 & 1 & 0 & 1 & 26 & 0 & 1 & n.b. & n.b. \\
\hline 5 & Tag 0 & rotierend & 1 & 1 & 0 & 1 & 26 & 0 & n.b. & 0,125 & 0,705 \\
\hline 5 & Tag 14 & rotierend & 1 & 1 & 0 & 1 & 26 & 0 & 0,839 & 0,042 & 0,571 \\
\hline 5 & Woche 8 & rotierend & 1 & 1 & 0 & 1 & 26 & 0 & 0,83 & 0,083 & 0,634 \\
\hline 5 & Woche 12 & rotierend & 1 & 1 & 0 & 1 & 26 & 0 & 0,863 & 0,083 & 0,589 \\
\hline 5 & Woche 16 & rotierend & 1 & 1 & 0 & 1 & 26 & 0 & 0,827 & 0,125 & 0,598 \\
\hline 5 & Woche 24 & rotierend & 1 & 1 & 0 & 1 & 26 & 0 & 1,077 & 0,208 & 0,866 \\
\hline 5 & Woche 25 & rotierend & 1 & 1 & 0 & 1 & 26 & 0 & 0,875 & 0,042 & 0,527 \\
\hline 6 & base & rotierend & 1 & 1 & 0 & 0 & 29 & 1 & 0,93 & n.b. & n.b. \\
\hline 6 & Tag 0 & rotierend & 1 & 1 & 0 & 0 & 29 & 1 & n.b. & 0,208 & 0,705 \\
\hline 6 & Tag 14 & rotierend & 1 & 1 & 0 & 0 & 29 & 1 & 0,577 & 0,167 & 0,482 \\
\hline 6 & Woche 8 & rotierend & 1 & 1 & 0 & 0 & 29 & 1 & 0,524 & 0,083 & 0,313 \\
\hline 6 & Woche 12 & rotierend & 1 & 1 & 0 & 0 & 29 & 1 & 0,702 & 0,042 & 0,509 \\
\hline 6 & Woche 16 & rotierend & 1 & 1 & 0 & 0 & 29 & 1 & 0,53 & 0,083 & 0,527 \\
\hline 6 & Woche 24 & rotierend & 1 & 1 & 0 & 0 & 29 & 1 & 0,923 & 0,125 & 0,554 \\
\hline 6 & Woche 25 & rotierend & 1 & 1 & 0 & 0 & 29 & 1 & 0,565 & 0,083 & 0,411 \\
\hline 7 & base & rotierend & 1 & 0 & 0 & 1 & 22 & 7 & 2,1 & n.b. & n.b. \\
\hline 7 & Tag 0 & rotierend & 1 & 0 & 0 & 1 & 22 & 7 & n.b. & 0,55 & 1,177 \\
\hline 7 & Tag 14 & rotierend & 1 & 0 & 0 & 1 & 22 & 7 & 1,451 & 0,4 & 1,01 \\
\hline 7 & Woche 8 & rotierend & 1 & 0 & 0 & 1 & 22 & 7 & 1,34 & 0,4 & 1,031 \\
\hline 7 & Woche 12 & rotierend & 1 & 0 & 0 & 1 & 22 & 7 & 1,306 & 0,45 & 1,052 \\
\hline 7 & Woche 16 & rotierend & 1 & 0 & 0 & 1 & 22 & 7 & 1,234 & 0,35 & 1,042 \\
\hline 7 & Woche 24 & rotierend & 1 & 0 & 0 & 1 & 22 & 7 & 1,438 & 0,55 & 1,313 \\
\hline 7 & Woche 25 & rotierend & 1 & 0 & 0 & 1 & 22 & 7 & 1,111 & 0,3 & 0,99 \\
\hline 8 & base & rotierend & 1 & 2 & 1 & 0 & 22 & 0 & 0,23 & n.b. & n.b. \\
\hline 8 & Tag 0 & rotierend & 1 & 2 & 1 & 0 & 22 & 0 & n.b. & 0,042 & 0,384 \\
\hline 8 & Tag 14 & rotierend & 1 & 2 & 1 & 0 & 22 & 0 & 0,22 & 0 & 0,304 \\
\hline 8 & Woche 8 & rotierend & 1 & 2 & 1 & 0 & 22 & 0 & 0,28 & 0 & 0,375 \\
\hline 8 & Woche 12 & rotierend & 1 & 2 & 1 & 0 & 22 & 0 & 0,28 & 0 & 0,348 \\
\hline 8 & Woche 16 & rotierend & 1 & 2 & 1 & 0 & 22 & 0 & 0,22 & 0,083 & 0,455 \\
\hline 8 & Woche 24 & rotierend & 1 & 2 & 1 & 0 & 22 & 0 & 0,381 & 0,25 & 0,696 \\
\hline 8 & Woche 25 & rotierend & 1 & 2 & 1 & 0 & 22 & 0 & 0,304 & 0 & 0,277 \\
\hline 9 & base & rotierend & 1 & 0 & 0 & 0 & 25 & 18 & 1,56 & n.b. & n.b. \\
\hline 9 & Tag 0 & rotierend & 1 & 0 & 0 & 0 & 25 & 18 & n.b. & 0,208 & 0,723 \\
\hline 9 & Tag 14 & rotierend & 1 & 0 & 0 & 0 & 25 & 18 & 0,589 & 0,167 & 0,5 \\
\hline 9 & Woche 8 & rotierend & 1 & 0 & 0 & 0 & 25 & 18 & 0,512 & 0,125 & 0,42 \\
\hline 9 & Woche 12 & rotierend & 1 & 0 & 0 & 0 & 25 & 18 & 0,696 & 0,083 & 0,482 \\
\hline 9 & Woche 16 & rotierend & 1 & 0 & 0 & 0 & 25 & 18 & 0,536 & 0,125 & 0,563 \\
\hline 9 & Woche 24 & rotierend & 1 & 0 & 0 & 0 & 25 & 18 & 0,911 & 0,25 & 0,679 \\
\hline 9 & Woche 25 & rotierend & 1 & 0 & 0 & 0 & 25 & 18 & 0,595 & 0,083 & 0,438 \\
\hline 10 & base & rotierend & 1 & 1 & 0 & 1 & 24 & 11 & 0,38 & n.b. & n.b. \\
\hline 10 & Tag 0 & rotierend & 1 & 1 & 0 & 1 & 24 & 11 & n.b. & 0,05 & 0,396 \\
\hline 10 & Tag 14 & rotierend & 1 & 1 & 0 & 1 & 24 & 11 & 0,682 & 0,05 & 0,323 \\
\hline 10 & Woche 8 & rotierend & 1 & 1 & 0 & 1 & 24 & 11 & 0,712 & 0,05 & 0,354 \\
\hline 10 & Woche 12 & rotierend & 1 & 1 & 0 & 1 & 24 & 11 & 0,53 & 0,05 & 0,365 \\
\hline 10 & Woche 16 & rotierend & 1 & 1 & 0 & 1 & 24 & 11 & 0,735 & 0,05 & 0,448 \\
\hline 10 & Woche 24 & rotierend & 1 & 1 & 0 & 1 & 24 & 11 & 1,09 & 0,2 & 0,594 \\
\hline
\end{tabular}




\begin{tabular}{|c|c|c|c|c|c|c|c|c|c|c|c|}
\hline 10 & Woche 25 & rotierend & 1 & 1 & 0 & 1 & 24 & 11 & 0,424 & 0,05 & 0,292 \\
\hline 11 & base & rotierend & 1 & 1 & 0 & 1 & 23 & 3 & 0,7 & n.b. & n.b. \\
\hline 11 & Tag 0 & rotierend & 1 & 1 & 0 & 1 & 23 & 3 & n.b. & 0,125 & 0,696 \\
\hline 11 & Tag 14 & rotierend & 1 & 1 & 0 & 1 & 23 & 3 & 0,863 & 0,083 & 0,616 \\
\hline 11 & Woche 8 & rotierend & 1 & 1 & 0 & 1 & 23 & 3 & 0,815 & 0,083 & 0,616 \\
\hline 11 & Woche 12 & rotierend & 1 & 1 & 0 & 1 & 23 & 3 & 0,839 & 0,125 & 0,616 \\
\hline 11 & Woche 16 & rotierend & 1 & 1 & 0 & 1 & 23 & 3 & 0,786 & 0,125 & 0,679 \\
\hline 11 & Woche 24 & rotierend & 1 & 1 & 0 & 1 & 23 & 3 & 1,155 & 0,292 & 0,92 \\
\hline 11 & Woche 25 & rotierend & 1 & 1 & 0 & 1 & 23 & 3 & 0,839 & 0,083 & 0,491 \\
\hline 12 & base & rotierend & 1 & 1 & 1 & 0 & 28 & 8 & 0,91 & n.b. & n.b. \\
\hline 12 & Tag 0 & rotierend & 1 & 1 & 1 & 0 & 28 & 8 & n.b. & 0,045 & 0,673 \\
\hline 12 & Tag 14 & rotierend & 1 & 1 & 1 & 0 & 28 & 8 & 0,944 & 0,136 & 0,519 \\
\hline 12 & Woche 8 & rotierend & 1 & 1 & 1 & 0 & 28 & 8 & 0,891 & 0,182 & 0,452 \\
\hline 12 & Woche 12 & rotierend & 1 & 1 & 1 & 0 & 28 & 8 & 0,917 & 0,182 & 0,571 \\
\hline 12 & Woche 16 & rotierend & 1 & 1 & 1 & 0 & 28 & 8 & 1,09 & 0,277 & 0,692 \\
\hline 12 & Woche 24 & rotierend & 1 & 1 & 1 & 0 & 28 & 8 & 1,372 & 0,455 & 1,048 \\
\hline 12 & Woche 25 & rotierend & 1 & 1 & 1 & 0 & 28 & 8 & 0,904 & 0,273 & 0,588 \\
\hline 13 & base & rotierend & 0 & 1 & 0 & 0 & 23 & 3 & 0,41 & n.b. & n.b. \\
\hline 13 & Tag 0 & rotierend & 0 & 1 & 0 & 1 & 23 & 3 & n.b. & 0,179 & 0,473 \\
\hline 13 & Tag 14 & rotierend & 0 & 1 & 0 & 1 & 23 & 3 & 0,417 & 0,143 & 0.313 \\
\hline 13 & Woche 8 & rotierend & 0 & 1 & 0 & 1 & 23 & 3 & 0,649 & 0,143 & 0,313 \\
\hline 13 & Woche 12 & rotierend & 0 & 1 & 0 & 1 & 23 & 3 & 1,536 & 0,5 & 0,402 \\
\hline 13 & Woche 16 & rotierend & 0 & 1 & 0 & 1 & 23 & 3 & 0,952 & 0,286 & 0,384 \\
\hline 13 & Woche 24 & rotierend & 0 & 1 & 0 & 1 & 23 & 3 & 0,327 & 0,107 & 0,446 \\
\hline 13 & Woche 25 & rotierend & 0 & 1 & 0 & 1 & 23 & 3 & 0,631 & 0,321 & 0,563 \\
\hline 14 & base & rotierend & 0 & 1 & 1 & 0 & 31 & 8 & 1,45 & n.b. & n.b. \\
\hline 14 & Tag 0 & rotierend & 0 & 1 & 1 & 0 & 31 & 8 & n.b. & 0,107 & 0,313 \\
\hline 14 & Tag 14 & rotierend & 0 & 1 & 1 & 0 & 31 & 8 & 0,857 & 0,107 & 0,393 \\
\hline 14 & Woche 8 & rotierend & 0 & 1 & 1 & 0 & 31 & 8 & 1,06 & 0,286 & 0,295 \\
\hline 14 & Woche 12 & rotierend & 0 & 1 & 1 & 0 & 31 & 8 & 1,553 & 0,071 & 0,545 \\
\hline 14 & Woche 16 & rotierend & 0 & 1 & 1 & 0 & 31 & 8 & 1,345 & 0,107 & 0,491 \\
\hline 14 & Woche 24 & rotierend & 0 & 1 & 1 & 0 & 31 & 8 & 1,44 & 0,25 & 0,402 \\
\hline 14 & Woche 25 & rotierend & 0 & 1 & 1 & 0 & 31 & 8 & 1,482 & 0,286 & 0,58 \\
\hline 15 & base & rotierend & 0 & 0 & 0 & 0 & 27 & 7 & 1,55 & n.b. & n.b. \\
\hline 15 & Tag 0 & rotierend & 0 & 0 & 0 & 0 & 27 & 7 & n.b. & 0,036 & 0,232 \\
\hline 15 & Tag 14 & rotierend & 0 & 0 & 0 & 0 & 27 & 7 & 1,036 & 0,036 & 0,527 \\
\hline 15 & Woche 8 & rotierend & 0 & 0 & 0 & 0 & 27 & 7 & 0,31 & 0,107 & 0,554 \\
\hline 15 & Woche 12 & rotierend & 0 & 0 & 0 & 0 & 27 & 7 & 0,565 & 0,071 & 0,455 \\
\hline 15 & Woche 16 & rotierend & 0 & 0 & 0 & 0 & 27 & 7 & 1,06 & 0,143 & 0,518 \\
\hline 15 & Woche 24 & rotierend & 0 & 0 & 0 & 0 & 27 & 7 & 0,827 & 0,143 & 0,518 \\
\hline 15 & Woche 25 & rotierend & 0 & 0 & 0 & 0 & 27 & 7 & 0,488 & 0,143 & 0,598 \\
\hline 16 & base & rotierend & 0 & 1 & 0 & 1 & 22 & 2 & 1,45 & n.b. & n.b. \\
\hline 16 & Tag 0 & rotierend & 0 & 1 & 0 & 1 & 22 & 2 & n.b. & 0,375 & 0,991 \\
\hline 16 & Tag 14 & rotierend & 0 & 1 & 0 & 1 & 22 & 2 & 0,815 & 0,708 & 0,955 \\
\hline 16 & Woche 8 & rotierend & 0 & 1 & 0 & 1 & 22 & 2 & 1,173 & 0,667 & 1,054 \\
\hline 16 & Woche 12 & rotierend & 0 & 1 & 0 & 1 & 22 & 2 & 1,054 & 0,167 & 0,982 \\
\hline 16 & Woche 16 & rotierend & 0 & 1 & 0 & 1 & 22 & 2 & 0,857 & 0,5 & 0,911 \\
\hline 16 & Woche 24 & rotierend & 0 & 1 & 0 & 1 & 22 & 2 & 1,06 & 0,5 & 0,946 \\
\hline 16 & Woche 25 & rotierend & 0 & 1 & 0 & 1 & 22 & 2 & 0,827 & 0,5 & 0,973 \\
\hline
\end{tabular}




\begin{tabular}{|c|c|c|c|c|c|c|c|c|c|c|c|}
\hline 17 & base & rotierend & 0 & 0 & 0 & 1 & 23 & 12 & 0,86 & n.b. & n.b. \\
\hline 17 & Tag 0 & rotierend & 0 & 0 & 0 & 1 & 23 & 12 & n.b. & 0,25 & 0,563 \\
\hline 17 & Tag 14 & rotierend & 0 & 0 & 0 & 1 & 23 & 12 & 0,78 & 0,167 & 0,536 \\
\hline 17 & Woche 8 & rotierend & 0 & 0 & 0 & 1 & 23 & 12 & 0,756 & 0,333 & 0,598 \\
\hline 17 & Woche 12 & rotierend & 0 & 0 & 0 & 1 & 23 & 12 & 0,768 & 0,542 & 0,527 \\
\hline 17 & Woche 16 & rotierend & 0 & 0 & 0 & 1 & 23 & 12 & 0,946 & 0,167 & 0,571 \\
\hline 17 & Woche 24 & rotierend & 0 & 0 & 0 & 1 & 23 & 12 & 0,768 & 0,208 & 0,563 \\
\hline 17 & Woche 25 & rotierend & 0 & 0 & 0 & 1 & 23 & 12 & 0,786 & 0,25 & 0,536 \\
\hline 18 & base & rotierend & 0 & 0 & 0 & 1 & 70 & 16 & 0,55 & n.b. & n.b. \\
\hline 18 & Tag 0 & rotierend & 0 & 0 & 0 & 1 & 70 & 16 & n.b. & 0,273 & 0,423 \\
\hline 18 & Tag 14 & rotierend & 0 & 0 & 0 & 1 & 70 & 16 & 0,398 & 0,182 & 0,431 \\
\hline 18 & Woche 8 & rotierend & 0 & 0 & 0 & 1 & 70 & 16 & 0,472 & 0,273 & 0,452 \\
\hline 18 & Woche 12 & rotierend & 0 & 0 & 0 & 1 & 70 & 16 & 0,398 & 0,136 & 0,404 \\
\hline 18 & Woche 16 & rotierend & 0 & 0 & 0 & 1 & 70 & 16 & 0,38 & 0,182 & 0,471 \\
\hline 18 & Woche 24 & rotierend & 0 & 0 & 0 & 1 & 70 & 16 & 0,5 & 0,273 & 0,375 \\
\hline 18 & Woche 25 & rotierend & 0 & 0 & 0 & 1 & 70 & 16 & 0,407 & 0,182 & 0,452 \\
\hline 19 & base & rotierend & 0 & 0 & 0 & 0 & 72 & 16 & 0,81 & n.b. & n.b. \\
\hline 19 & Tag 0 & rotierend & 0 & 0 & 0 & 0 & 72 & 16 & n.b. & 0,238 & 0,65 \\
\hline 19 & Tag 14 & rotierend & 0 & 0 & 0 & 0 & 72 & 16 & 0,771 & 0,238 & 0,59 \\
\hline 19 & Woche 8 & rotierend & 0 & 0 & 0 & 0 & 72 & 16 & 0,906 & 0,286 & 0,63 \\
\hline 19 & Woche 12 & rotierend & 0 & 0 & 0 & 0 & 72 & 16 & 0,83 & 0,19 & 0,64 \\
\hline 19 & Woche 16 & rotierend & 0 & 0 & 0 & 0 & 72 & 16 & 0,813 & 0,19 & 0,66 \\
\hline 19 & Woche 24 & rotierend & 0 & 0 & 0 & 0 & 72 & 16 & 0,75 & 0,19 & 0,7 \\
\hline 19 & Woche 25 & rotierend & 0 & 0 & 0 & 0 & 72 & 16 & 0,719 & 0,143 & 0,68 \\
\hline 20 & base & rotierend & 0 & 0 & 0 & 1 & 25 & 8 & 0,21 & n.b. & n.b. \\
\hline 20 & Tag 0 & rotierend & 0 & 0 & 0 & 1 & 25 & 8 & n.b. & 0,125 & 0,214 \\
\hline 20 & Tag 14 & rotierend & 0 & 0 & 0 & 1 & 25 & 8 & 0,194 & 0,083 & 0,179 \\
\hline 20 & Woche 8 & rotierend & 0 & 0 & 0 & 1 & 25 & 8 & 0,238 & 0,083 & 0,205 \\
\hline 20 & Woche 12 & rotierend & 0 & 0 & 0 & 1 & 25 & 8 & 0,185 & 0,042 & 0,17 \\
\hline 20 & Woche 16 & rotierend & 0 & 0 & 0 & 1 & 25 & 8 & 0,196 & 0,083 & 0,17 \\
\hline 20 & Woche 24 & rotierend & 0 & 0 & 0 & 1 & 25 & 8 & 0,179 & 0,042 & 0,188 \\
\hline 20 & Woche 25 & rotierend & 0 & 0 & 0 & 1 & 25 & 8 & 0,167 & 0,042 & 0,161 \\
\hline 21 & base & rotierend & 0 & 0 & 1 & 0 & 30 & 13 & 1,42 & n.b. & n.b. \\
\hline 21 & Tag 0 & rotierend & 0 & 0 & 1 & 0 & 30 & 13 & n.b. & 0,391 & 0,657 \\
\hline 21 & Tag 14 & rotierend & 0 & 0 & 1 & 0 & 30 & 13 & 0,433 & 0,261 & 0,565 \\
\hline 21 & Woche 8 & rotierend & 0 & 0 & 1 & 0 & 30 & 13 & 0,807 & 0,261 & 0,63 \\
\hline 21 & Woche 12 & rotierend & 0 & 0 & 1 & 0 & 30 & 13 & 0,633 & 0,304 & 0,676 \\
\hline 21 & Woche 16 & rotierend & 0 & 0 & 1 & 0 & 30 & 13 & 0,473 & 0,348 & 0,704 \\
\hline 21 & Woche 24 & rotierend & 0 & 0 & 1 & 0 & 30 & 13 & 0,851 & 0,261 & 0,75 \\
\hline 21 & Woche 25 & rotierend & 0 & 0 & 1 & 0 & 30 & 13 & 0,5 & 0,304 & 0,527 \\
\hline 22 & base & rotierend & 0 & 0 & 1 & 0 & 22 & 9 & 0,25 & n.b. & n.b. \\
\hline 22 & Tag 0 & rotierend & 0 & 0 & 1 & 0 & 22 & 9 & n.b. & 0,5 & 0,839 \\
\hline 22 & Tag 14 & rotierend & 0 & 0 & 1 & 0 & 22 & 9 & 0,226 & 0,333 & 0,616 \\
\hline 22 & Woche 8 & rotierend & 0 & 0 & 1 & 0 & 22 & 9 & 0,256 & 0,375 & 0,643 \\
\hline 22 & Woche 12 & rotierend & 0 & 0 & 1 & 0 & 22 & 9 & 0,232 & 0,292 & 0,634 \\
\hline 22 & Woche 16 & rotierend & 0 & 0 & 1 & 0 & 22 & 9 & 0,214 & 0,25 & 0,626 \\
\hline 22 & Woche 24 & rotierend & 0 & 0 & 1 & 0 & 22 & 9 & 0,173 & 0,292 & 0,67 \\
\hline 22 & Woche 25 & rotierend & 0 & 0 & 1 & 0 & 22 & 9 & 0,208 & 0,25 & 0,598 \\
\hline 23 & base & rotierend & 0 & 1 & 0 & 1 & 25 & 6 & 1,31 & n.b. & n.b. \\
\hline
\end{tabular}




\begin{tabular}{|c|c|c|c|c|c|c|c|c|c|c|c|}
\hline 23 & Tag 0 & rotierend & 0 & 1 & 0 & 1 & 25 & 6 & n.b. & 0,6 & 1,021 \\
\hline 23 & Tag 14 & rotierend & 0 & 1 & 0 & 1 & 25 & 6 & 0,903 & 0,5 & 0,99 \\
\hline 23 & Woche 8 & rotierend & 0 & 1 & 0 & 1 & 25 & 6 & 0,889 & 0,45 & 0,927 \\
\hline 23 & Woche 12 & rotierend & 0 & 1 & 0 & 1 & 25 & 6 & 0,938 & 0,55 & 0,938 \\
\hline 23 & Woche 16 & rotierend & 0 & 1 & 0 & 1 & 25 & 6 & 0,896 & 0,6 & 0,917 \\
\hline 23 & Woche 24 & rotierend & 0 & 1 & 0 & 1 & 25 & 6 & 0,965 & 0,45 & 0,927 \\
\hline 23 & Woche 25 & rotierend & 0 & 1 & 0 & 1 & 25 & 6 & 0,889 & 0,5 & 0,844 \\
\hline 24 & base & rotierend & 0 & 1 & 0 & 1 & 32 & 5 & 0,32 & n.b. & n.b. \\
\hline 24 & Tag 0 & rotierend & 0 & 1 & 0 & 1 & 32 & 5 & n.b. & 0,208 & 0,455 \\
\hline 24 & Tag 14 & rotierend & 0 & 1 & 0 & 1 & 32 & 5 & 0,272 & 0,125 & 0,491 \\
\hline 24 & Woche 8 & rotierend & 0 & 1 & 0 & 1 & 32 & 5 & 0,315 & 0,125 & 0,42 \\
\hline 24 & Woche 12 & rotierend & 0 & 1 & 0 & 1 & 32 & 5 & 0,37 & 0,167 & 0,545 \\
\hline 24 & Woche 16 & rotierend & 0 & 1 & 0 & 1 & 32 & 5 & 0,302 & 0,125 & 0,51 \\
\hline 24 & Woche 24 & rotierend & 0 & 1 & 0 & 1 & 32 & 5 & 0,21 & 0,083 & 0,384 \\
\hline 24 & Woche 25 & rotierend & 0 & 1 & 0 & 1 & 32 & 5 & 0,302 & 0 & 0,464 \\
\hline 25 & base & Schall & 1 & 1 & 0 & 1 & 24 & 13 & 0,14 & n.b. & n.b. \\
\hline 25 & Tag 0 & Schall & 1 & 1 & 0 & 1 & 24 & 13 & n.b. & 0,042 & 0,125 \\
\hline 25 & Tag 14 & Schall & 1 & 1 & 0 & 1 & 24 & 13 & n.b. & n.b. & n.b. \\
\hline 25 & Woche 8 & Schall & 1 & 1 & 0 & 1 & 24 & 13 & 0,475 & 0 & 0,179 \\
\hline 25 & Woche 12 & Schall & 1 & 1 & 0 & 1 & 24 & 13 & 0,51 & 0 & 0,464 \\
\hline 25 & Woche 16 & Schall & 1 & 1 & 0 & 1 & 24 & 13 & 0,488 & 0,083 & 0,304 \\
\hline 25 & Woche 24 & Schall & 1 & 1 & 0 & 1 & 24 & 13 & 0,802 & 0,042 & 0,589 \\
\hline 25 & Woche 25 & Schall & 1 & 1 & 0 & 1 & 24 & 13 & 0,5 & 0 & 0,438 \\
\hline 26 & base & Schall & 1 & 1 & 0 & 1 & 24 & 10 & 0,5 & n.b. & n.b. \\
\hline 26 & Tag 0 & Schall & 1 & 1 & 0 & 1 & 24 & 10 & n.b. & 0,208 & 0,5 \\
\hline 26 & Tag 14 & Schall & 1 & 1 & 0 & 1 & 24 & 10 & 0,732 & 0,042 & 0,411 \\
\hline 26 & Woche 8 & Schall & 1 & 1 & 0 & 1 & 24 & 10 & 0,411 & 0,208 & 0,402 \\
\hline 26 & Woche 12 & Schall & 1 & 1 & 0 & 1 & 24 & 10 & 0,741 & 0,25 & 0,402 \\
\hline 26 & Woche 16 & Schall & 1 & 1 & 0 & 1 & 24 & 10 & 0,786 & 0,167 & 0,661 \\
\hline 26 & Woche 24 & Schall & 1 & 1 & 0 & 1 & 24 & 10 & 0,53 & 0,083 & 0,732 \\
\hline 26 & Woche 25 & Schall & 1 & 1 & 0 & 1 & 24 & 10 & 0,649 & 0,083 & 0,545 \\
\hline 27 & base & Schall & 1 & 0 & 0 & 1 & 23 & 2 & 0,71 & n.b. & n.b. \\
\hline 27 & Tag 0 & Schall & 1 & 0 & 0 & 1 & 23 & 2 & n.b. & 0,435 & 0,657 \\
\hline 27 & Tag 14 & Schall & 1 & 0 & 0 & 1 & 23 & 2 & 0,593 & 0,043 & 0,537 \\
\hline 27 & Woche 8 & Schall & 1 & 0 & 0 & 1 & 23 & 2 & 0,352 & 0,043 & 0,593 \\
\hline 27 & Woche 12 & Schall & 1 & 0 & 0 & 1 & 23 & 2 & 0,346 & 0,087 & 0,426 \\
\hline 27 & Woche 16 & Schall & 1 & 0 & 0 & 1 & 23 & 2 & 0,599 & 0,391 & 0,5 \\
\hline 27 & Woche 24 & Schall & 1 & 0 & 0 & 1 & 23 & 2 & 0,88 & 0,087 & 0,991 \\
\hline 27 & Woche 25 & Schall & 1 & 0 & 0 & 1 & 23 & 2 & 0,562 & 0,277 & 0,546 \\
\hline 28 & base & Schall & 1 & 1 & 1 & 0 & 24 & 2 & 1,41 & n.b. & n.b. \\
\hline 28 & Tag 0 & Schall & 1 & 1 & 1 & 0 & 24 & 2 & n.b. & 0,286 & 1,071 \\
\hline 28 & Tag 14 & Schall & 1 & 1 & 1 & 0 & 24 & 2 & 1,22 & 0,107 & 0,813 \\
\hline 28 & Woche 8 & Schall & 1 & 1 & 1 & 0 & 24 & 2 & 1,185 & 0,107 & 0,83 \\
\hline 28 & Woche 12 & Schall & 1 & 1 & 1 & 0 & 24 & 2 & n.b. & n.b. & n.b. \\
\hline 28 & Woche 16 & Schall & 1 & 1 & 1 & 0 & 24 & 2 & 1,268 & 0,143 & 0,902 \\
\hline 28 & Woche 24 & Schall & 1 & 1 & 1 & 0 & 24 & 2 & 1,821 & 0,393 & 0,973 \\
\hline 28 & Woche 25 & Schall & 1 & 1 & 1 & 0 & 24 & 2 & 1,19 & 0,25 & 0,875 \\
\hline 29 & base & Schall & 1 & 1 & 1 & 0 & 28 & 7 & 0,95 & n.b. & n.b. \\
\hline 29 & Tag 0 & Schall & 1 & 1 & 1 & 0 & 28 & 7 & n.b. & 0,333 & 0,83 \\
\hline
\end{tabular}




\begin{tabular}{|c|c|c|c|c|c|c|c|c|c|c|c|}
\hline 29 & Tag 14 & Schall & 1 & 1 & 1 & 0 & 28 & 7 & 0,78 & 0,25 & 0,786 \\
\hline 29 & Woche 8 & Schall & 1 & 1 & 1 & 0 & 28 & 7 & 0,786 & 0,25 & 0,813 \\
\hline 29 & Woche 12 & Schall & 1 & 1 & 1 & 0 & 28 & 7 & 0,893 & 0,208 & 0,81 \\
\hline 29 & Woche 16 & Schall & 1 & 1 & 1 & 0 & 28 & 7 & 0,875 & 0,25 & 0,795 \\
\hline 29 & Woche 24 & Schall & 1 & 1 & 1 & 0 & 28 & 7 & 0,988 & 0,292 & 0,848 \\
\hline 29 & Woche 25 & Schall & 1 & 1 & 1 & 0 & 28 & 7 & 0,887 & 0,208 & 0,813 \\
\hline 30 & base & Schall & 1 & 1 & 0 & 0 & 28 & 2 & 1,73 & n.b. & n.b. \\
\hline 30 & Tag 0 & Schall & 1 & 1 & 0 & 0 & 28 & 2 & n.b. & 0,375 & 0,991 \\
\hline 30 & Tag 14 & Schall & 1 & 1 & 0 & 0 & 28 & 2 & 0,97 & 0,333 & 0,893 \\
\hline 30 & Woche 8 & Schall & 1 & 1 & 0 & 0 & 28 & 2 & 0,994 & 0,333 & 0,982 \\
\hline 30 & Woche 12 & Schall & 1 & 1 & 0 & 0 & 28 & 2 & 1,018 & 0,292 & 0,973 \\
\hline 30 & Woche 16 & Schall & 1 & 1 & 0 & 0 & 28 & 2 & 1,042 & 0,333 & 0,946 \\
\hline 30 & Woche 24 & Schall & 1 & 1 & 0 & 0 & 28 & 2 & 1,065 & 0,333 & 1,152 \\
\hline 30 & Woche 25 & Schall & 1 & 1 & 0 & 0 & 28 & 2 & 0,929 & 0,25 & 1,009 \\
\hline 31 & base & Schall & 1 & 1 & 1 & 1 & 27 & 4 & 0,46 & n.b. & n.b. \\
\hline 31 & Tag 0 & Schall & 1 & 1 & 1 & 1 & 27 & 4 & n.b. & 0,083 & 0,384 \\
\hline 31 & Tag 14 & Schall & 1 & 1 & 1 & 1 & 27 & 4 & 0,339 & 0,042 & 0,286 \\
\hline 31 & Woche 8 & Schall & 1 & 1 & 1 & 1 & 27 & 4 & 0,399 & 0,083 & 0,339 \\
\hline 31 & Woche 12 & Schall & 1 & 1 & 1 & 1 & 27 & 4 & 0,387 & 0,042 & 0,295 \\
\hline 31 & Woche 16 & Schall & 1 & 1 & 1 & 1 & 27 & 4 & 0,393 & 0 & 0,304 \\
\hline 31 & Woche 24 & Schall & 1 & 1 & 1 & 1 & 27 & 4 & 0,482 & 0,125 & 0,402 \\
\hline 31 & Woche 25 & Schall & 1 & 1 & 1 & 1 & 27 & 4 & 0,333 & 0 & 0,232 \\
\hline 32 & base & Schall & 1 & 1 & 0 & 1 & 32 & 10 & 0,38 & n.b. & n.b. \\
\hline 32 & Tag 0 & Schall & 1 & 1 & 0 & 1 & 32 & 10 & n.b. & 0,25 & 0,438 \\
\hline 32 & Tag 14 & Schall & 1 & 1 & 0 & 1 & 32 & 10 & 0,232 & 0,167 & 0,375 \\
\hline 32 & Woche 8 & Schall & 1 & 1 & 0 & 1 & 32 & 10 & 0,31 & 0,167 & 0,51 \\
\hline 32 & Woche 12 & Schall & 1 & 1 & 0 & 1 & 32 & 10 & 0,286 & 0,125 & 0,339 \\
\hline 32 & Woche 16 & Schall & 1 & 1 & 0 & 1 & 32 & 10 & 0,339 & 0,167 & 0,402 \\
\hline 32 & Woche 24 & Schall & 1 & 1 & 0 & 1 & 32 & 10 & 0,47 & 0,292 & 0,563 \\
\hline 32 & Woche 25 & Schall & 1 & 1 & 0 & 1 & 32 & 10 & 0,333 & 0,208 & 0,455 \\
\hline 33 & base & Schall & 1 & 0 & 0 & 1 & 28 & 9 & 0,88 & n.b. & n.b. \\
\hline 33 & Tag 0 & Schall & 1 & 0 & 0 & 1 & 28 & 9 & n.b. & 0,615 & 1,063 \\
\hline 33 & Tag 14 & Schall & 1 & 0 & 0 & 1 & 28 & 9 & 0,611 & 0,385 & 0,92 \\
\hline 33 & Woche 8 & Schall & 1 & 0 & 0 & 1 & 28 & 9 & 0,643 & 0,346 & 0,884 \\
\hline 33 & Woche 12 & Schall & 1 & 0 & 0 & 1 & 28 & 9 & 0,706 & 0,308 & 0,777 \\
\hline 33 & Woche 16 & Schall & 1 & 0 & 0 & 1 & 28 & 9 & 0,81 & 0,385 & 0,964 \\
\hline 33 & Woche 24 & Schall & 1 & 0 & 0 & 1 & 28 & 9 & 1,198 & 0,577 & 1,152 \\
\hline 33 & Woche 25 & Schall & 1 & 0 & 0 & 1 & 28 & 9 & 0,794 & 0,423 & 0,866 \\
\hline 34 & base & Schall & 1 & 1 & 1 & 1 & 28 & 4 & 2 & n.b. & n.b. \\
\hline 34 & Tag 0 & Schall & 1 & 1 & 1 & 1 & 28 & 4 & n.b. & 0,667 & 1,688 \\
\hline 34 & Tag 14 & Schall & 1 & 1 & 1 & 1 & 28 & 4 & 1,435 & 0,5 & 1,429 \\
\hline 34 & Woche 8 & Schall & 1 & 1 & 1 & 1 & 28 & 4 & 1,381 & 0,333 & 1,321 \\
\hline 34 & Woche 12 & Schall & 1 & 1 & 1 & 1 & 28 & 4 & 1,292 & 0,25 & 1,152 \\
\hline 34 & Woche 16 & Schall & 1 & 1 & 1 & 1 & 28 & 4 & 1,363 & 0,375 & 1,402 \\
\hline 34 & Woche 24 & Schall & 1 & 1 & 1 & 1 & 28 & 4 & 1,494 & 0,542 & 1,527 \\
\hline 34 & Woche 25 & Schall & 1 & 1 & 1 & 1 & 28 & 4 & 1,298 & 0,458 & 1,313 \\
\hline 35 & base & Schall & 1 & 1 & 1 & 0 & 26 & 7 & 1,29 & n.b. & n.b. \\
\hline 35 & Tag 0 & Schall & 1 & 1 & 1 & 0 & 26 & 7 & n.b. & 0,458 & 0,679 \\
\hline 35 & Tag 14 & Schall & 1 & 1 & 1 & 0 & 26 & 7 & 0,643 & 0,25 & 0,554 \\
\hline
\end{tabular}




\begin{tabular}{|c|c|c|c|c|c|c|c|c|c|c|c|}
\hline 35 & Woche 8 & Schall & 1 & 1 & 1 & 0 & 26 & 7 & 0,375 & 0,167 & 0,705 \\
\hline 35 & Woche 12 & Schall & 1 & 1 & 1 & 0 & 26 & 7 & 0,399 & 0,125 & 0,455 \\
\hline 35 & Woche 16 & Schall & 1 & 1 & 1 & 0 & 26 & 7 & 0,577 & 0,333 & 0,598 \\
\hline 35 & Woche 24 & Schall & 1 & 1 & 1 & 0 & 26 & 7 & 0,935 & 0,375 & 1,098 \\
\hline 35 & Woche 25 & Schall & 1 & 1 & 1 & 0 & 26 & 7 & 0,53 & 0,208 & 0,741 \\
\hline 36 & base & Schall & 1 & 1 & 0 & 0 & 26 & 10 & 0,2 & n.b. & n.b. \\
\hline 36 & Tag 0 & Schall & 1 & 1 & 0 & 0 & 26 & 10 & n.b. & 0,042 & 0,366 \\
\hline 36 & Tag 14 & Schall & 1 & 1 & 0 & 0 & 26 & 10 & 0,28 & 0,042 & 0,196 \\
\hline 36 & Woche 8 & Schall & 1 & 1 & 0 & 0 & 26 & 10 & 0,446 & 0,042 & 0,214 \\
\hline 36 & Woche 12 & Schall & 1 & 1 & 0 & 0 & 26 & 10 & 0,387 & 0,083 & 0,393 \\
\hline 36 & Woche 16 & Schall & 1 & 1 & 0 & 0 & 26 & 10 & 0,333 & 0,042 & 0,339 \\
\hline 36 & Woche 24 & Schall & 1 & 1 & 0 & 0 & 26 & 10 & 0,435 & 0,167 & 0,571 \\
\hline 36 & Woche 25 & Schall & 1 & 1 & 0 & 0 & 26 & 10 & 0,28 & 0,042 & 0,205 \\
\hline 37 & base & Schall & 0 & 1 & 0 & 1 & 24 & 3 & 0,79 & n.b. & n.b. \\
\hline 37 & Tag 0 & Schall & 0 & 1 & 0 & 1 & 24 & 3 & n.b. & 0,042 & 0,58 \\
\hline 37 & Tag 14 & Schall & 0 & 1 & 0 & 1 & 24 & 3 & 0,661 & 0,25 & 0,429 \\
\hline 37 & Woche 8 & Schall & 0 & 1 & 0 & 1 & 24 & 3 & 0,714 & 0,25 & 0,51 \\
\hline 37 & Woche 12 & Schall & 0 & 1 & 0 & 1 & 24 & 3 & 0,696 & 0,167 & 0,545 \\
\hline 37 & Woche 16 & Schall & 0 & 1 & 0 & 1 & 24 & 3 & 0,732 & 0,25 & 0,527 \\
\hline 37 & Woche 24 & Schall & 0 & 1 & 0 & 1 & 24 & 3 & 0,708 & 0,208 & 0,473 \\
\hline 37 & Woche 25 & Schall & 0 & 1 & 0 & 1 & 24 & 3 & 0,679 & 0,125 & 0,563 \\
\hline 38 & base & Schall & 0 & 1 & 0 & 1 & 29 & 10 & 0,29 & n.b. & n.b. \\
\hline 38 & Tag 0 & Schall & 0 & 1 & 0 & 1 & 29 & 10 & n.b. & 0,083 & 0,571 \\
\hline 38 & Tag 14 & Schall & 0 & 1 & 0 & 1 & 29 & 10 & 0,399 & 0,042 & 0,438 \\
\hline 38 & Woche 8 & Schall & 0 & 1 & 0 & 1 & 29 & 10 & 0,5 & 0,125 & 0,607 \\
\hline 38 & Woche 12 & Schall & 0 & 1 & 0 & 1 & 29 & 10 & 0,565 & 0,167 & 0,571 \\
\hline 38 & Woche 16 & Schall & 0 & 1 & 0 & 1 & 29 & 10 & 0,577 & 0,25 & 0,616 \\
\hline 38 & Woche 24 & Schall & 0 & 1 & 0 & 1 & 29 & 10 & 0,565 & 0,208 & 0,58 \\
\hline 38 & Woche 25 & Schall & 0 & 1 & 0 & 1 & 29 & 10 & 0,476 & 0,083 & 0,527 \\
\hline 39 & base & Schall & 0 & 1 & 0 & 1 & 31 & 15 & 1,25 & n.b. & n.b. \\
\hline 39 & Tag 0 & Schall & 0 & 1 & 0 & 1 & 31 & 15 & n.b. & 0,083 & 0,723 \\
\hline 39 & Tag 14 & Schall & 0 & 1 & 0 & 1 & 31 & 15 & 0,97 & 0,167 & 0,616 \\
\hline 39 & Woche 8 & Schall & 0 & 1 & 0 & 1 & 31 & 15 & 1,53 & 0,083 & 0,643 \\
\hline 39 & Woche 12 & Schall & 0 & 1 & 0 & 1 & 31 & 15 & 0,947 & 0,167 & 0,68 \\
\hline 39 & Woche 16 & Schall & 0 & 1 & 0 & 1 & 31 & 15 & 0,924 & 0,083 & 0,589 \\
\hline 39 & Woche 24 & Schall & 0 & 1 & 0 & 1 & 31 & 15 & 0,894 & 0,167 & 0,688 \\
\hline 39 & Woche 25 & Schall & 0 & 1 & 0 & 1 & 31 & 15 & 0,917 & 0,125 & 0,723 \\
\hline 40 & base & Schall & 0 & 0 & 0 & 0 & 24 & 1 & 1,07 & n.b. & n.b. \\
\hline 40 & Tag 0 & Schall & 0 & 0 & 0 & 0 & 24 & 1 & n.b. & 0,321 & 0,58 \\
\hline 40 & Tag 14 & Schall & 0 & 0 & 0 & 0 & 24 & 1 & 0,536 & 0,179 & 0,393 \\
\hline 40 & Woche 8 & Schall & 0 & 0 & 0 & 0 & 24 & 1 & 0,286 & 0,214 & 0,536 \\
\hline 40 & Woche 12 & Schall & 0 & 0 & 0 & 0 & 24 & 1 & n.b. & n.b. & n.b. \\
\hline 40 & Woche 16 & Schall & 0 & 0 & 0 & 0 & 24 & 1 & 0,601 & 0,286 & 0,589 \\
\hline 40 & Woche 24 & Schall & 0 & 0 & 0 & 0 & 24 & 1 & 1,298 & 0,393 & 0,527 \\
\hline 40 & Woche 25 & Schall & 0 & 0 & 0 & 0 & 24 & 1 & 0,631 & 0,25 & 0,42 \\
\hline 41 & base & Schall & 0 & 1 & 0 & 1 & 30 & 17 & 0,86 & n.b. & n.b. \\
\hline 41 & Tag 0 & Schall & 0 & 1 & 0 & 1 & 30 & 17 & n.b. & 0,25 & 0,723 \\
\hline 41 & Tag 14 & Schall & 0 & 1 & 0 & 1 & 30 & 17 & 0,744 & 0,167 & 0,616 \\
\hline 41 & Woche 8 & Schall & 0 & 1 & 0 & 1 & 30 & 17 & 0,72 & 0,208 & \\
\hline
\end{tabular}




\begin{tabular}{|c|c|c|c|c|c|c|c|c|c|c|c|}
\hline 41 & Woche 12 & Schall & 0 & 1 & 0 & 1 & 30 & 17 & 0,696 & 0,167 & 0,616 \\
\hline 41 & Woche 16 & Schall & 0 & 1 & 0 & 1 & 30 & 17 & 0,452 & 0,167 & 0,643 \\
\hline 41 & Woche 24 & Schall & 0 & 1 & 0 & 1 & 30 & 17 & 0,756 & 0,208 & 0,688 \\
\hline 41 & Woche 25 & Schall & 0 & 1 & 0 & 1 & 30 & 17 & 0,613 & 0,208 & 0,714 \\
\hline 42 & base & Schall & 0 & 1 & 0 & 1 & 26 & 12 & 2,08 & n.b. & n.b. \\
\hline 42 & Tag 0 & Schall & 0 & 1 & 0 & 1 & 26 & 12 & n.b. & 0,542 & 0,635 \\
\hline 42 & Tag 14 & Schall & 0 & 1 & 0 & 1 & 26 & 12 & 1,146 & 0,333 & 0,615 \\
\hline 42 & Woche 8 & Schall & 0 & 1 & 0 & 1 & 26 & 12 & 1,132 & 0,375 & 0,635 \\
\hline 42 & Woche 12 & Schall & 0 & 1 & 0 & 1 & 26 & 12 & 1,083 & 0,292 & 0,594 \\
\hline 42 & Woche 16 & Schall & 0 & 1 & 0 & 1 & 26 & 12 & 1,021 & 0,25 & 0,646 \\
\hline 42 & Woche 24 & Schall & 0 & 1 & 0 & 1 & 26 & 12 & 0,951 & 0,25 & 0,531 \\
\hline 42 & Woche 25 & Schall & 0 & 1 & 0 & 1 & 26 & 12 & 0,965 & 0,208 & 0,625 \\
\hline 43 & base & Schall & 0 & 2 & 0 & 1 & 21 & 2 & 1,5 & n.b. & n.b. \\
\hline 43 & Tag 0 & Schall & 0 & 2 & 0 & 1 & 21 & 2 & n.b. & 0,65 & 0,969 \\
\hline 43 & Tag 14 & Schall & 0 & 2 & 0 & 1 & 21 & 2 & 1,333 & 0,55 & 1,042 \\
\hline 43 & Woche 8 & Schall & 0 & 2 & 0 & 1 & 21 & 2 & 1,41 & 0,8 & 1,333 \\
\hline 43 & Woche 12 & Schall & 0 & 2 & 0 & 1 & 21 & 2 & 1,507 & 0,4 & 1,375 \\
\hline 43 & Woche 16 & Schall & 0 & 2 & 0 & 1 & 21 & 2 & 1,083 & 0,2 & 1,344 \\
\hline 43 & Woche 24 & Schall & 0 & 2 & 0 & 1 & 21 & 2 & 1,007 & 0,45 & 1,469 \\
\hline 43 & Woche 25 & Schall & 0 & 2 & 0 & 1 & 21 & 2 & 0,785 & 0,35 & 1,344 \\
\hline 44 & base & Schall & 0 & 1 & 0 & 0 & 41 & 15 & 0,7 & n.b. & n.b. \\
\hline 44 & Tag 0 & Schall & 0 & 1 & 0 & 0 & 41 & 15 & n.b. & 0,25 & 0,723 \\
\hline 44 & Tag 14 & Schall & 0 & 1 & 0 & 0 & 41 & 15 & 0,5 & 0,167 & 0,688 \\
\hline 44 & Woche 8 & Schall & 0 & 1 & 0 & 0 & 41 & 15 & 0,725 & 0,167 & 0,732 \\
\hline 44 & Woche 12 & Schall & 0 & 1 & 0 & 0 & 41 & 15 & 0,703 & 0,208 & 0,795 \\
\hline 44 & Woche 16 & Schall & 0 & 1 & 0 & 0 & 41 & 15 & 0,623 & 0,125 & 0,705 \\
\hline 44 & Woche 24 & Schall & 0 & 1 & 0 & 0 & 41 & 15 & 0,775 & 0,25 & 0,92 \\
\hline 44 & Woche 25 & Schall & 0 & 1 & 0 & 0 & 41 & 15 & 0,717 & 0,167 & 0,821 \\
\hline 45 & base & Schall & 0 & 1 & 0 & 1 & 38 & 12 & 1,89 & n.b. & n.b. \\
\hline 45 & Tag 0 & Schall & 0 & 1 & 0 & 1 & 38 & 12 & n.b. & 0,522 & 0,852 \\
\hline 45 & Tag 14 & Schall & 0 & 1 & 0 & 1 & 38 & 12 & 1,556 & 0,304 & 0,741 \\
\hline 45 & Woche 8 & Schall & 0 & 1 & 0 & 1 & 38 & 12 & 1,488 & 0,261 & 0,769 \\
\hline 45 & Woche 12 & Schall & 0 & 1 & 0 & 1 & 38 & 12 & 1,475 & 0,217 & 0,722 \\
\hline 45 & Woche 16 & Schall & 0 & 1 & 0 & 1 & 38 & 12 & 1,407 & 0,261 & 0,769 \\
\hline 45 & Woche 24 & Schall & 0 & 1 & 0 & 1 & 38 & 12 & 1,451 & 0,261 & 0,815 \\
\hline 45 & Woche 25 & Schall & 0 & 1 & 0 & 1 & 38 & 12 & 1,488 & 0,217 & 0,87 \\
\hline 46 & base & Schall & 0 & 1 & 0 & 1 & 52 & 21 & 0,173 & n.b. & n.b. \\
\hline 46 & Tag 0 & Schall & 0 & 1 & 0 & 1 & 52 & 21 & n.b. & 0,042 & 0,455 \\
\hline 46 & Tag 14 & Schall & 0 & 1 & 0 & 1 & 52 & 21 & 0,135 & 0 & 0,438 \\
\hline 46 & Woche 8 & Schall & 0 & 1 & 0 & 1 & 52 & 21 & 0,359 & 0,042 & 0,491 \\
\hline 46 & Woche 12 & Schall & 0 & 1 & 0 & 1 & 52 & 21 & 0,404 & 0,083 & 0,42 \\
\hline 46 & Woche 16 & Schall & 0 & 1 & 0 & 1 & 52 & 21 & 0,423 & 0 & 0,438 \\
\hline 46 & Woche 24 & Schall & 0 & 1 & 0 & 1 & 52 & 21 & 0,404 & 0,042 & 0,482 \\
\hline 46 & Woche 25 & Schall & 0 & 1 & 0 & 1 & 52 & 21 & 0,429 & 0 & 0,446 \\
\hline 47 & base & Schall & 0 & 2 & 0 & 0 & 23 & 8 & 1,11 & n.b. & n.b. \\
\hline 47 & Tag 0 & Schall & 0 & 2 & 0 & 0 & 23 & 8 & n.b. & 0,083 & 0,5 \\
\hline 47 & Tag 14 & Schall & 0 & 2 & 0 & 0 & 23 & 8 & 0,916 & 0,125 & 0,464 \\
\hline 47 & Woche 8 & Schall & 0 & 2 & 0 & 0 & 23 & 8 & 0,726 & 0,208 & 0,554 \\
\hline 47 & Woche 12 & Schall & 0 & 2 & 0 & 0 & 23 & 8 & 0,726 & 0,167 & 0,527 \\
\hline
\end{tabular}




\begin{tabular}{|c|c|c|c|c|c|c|c|c|c|c|c|}
\hline 47 & Woche 16 & Schall & 0 & 2 & 0 & 0 & 23 & 8 & 0,887 & 0,16 & 0,51 \\
\hline 47 & Woche 24 & Schall & 0 & 2 & 0 & 0 & 23 & 8 & 0,815 & 0,083 & 0,473 \\
\hline 47 & Woche 25 & Schall & 0 & 2 & 0 & 0 & 23 & 8 & 0,732 & 0,083 & 0,554 \\
\hline 48 & base & Schall & 0 & 0 & 0 & 1 & 23 & 8 & 0,89 & n.b. & n.b. \\
\hline 48 & Tag 0 & Schall & 0 & 0 & 0 & 1 & 23 & 8 & n.b. & 0,583 & 0,821 \\
\hline 48 & Tag 14 & Schall & 0 & 0 & 0 & 1 & 23 & 8 & 1,185 & 0,25 & 0,527 \\
\hline 48 & Woche 8 & Schall & 0 & 0 & 0 & 1 & 23 & 8 & 0,661 & 0,167 & 0,589 \\
\hline 48 & Woche 12 & Schall & 0 & 0 & 0 & 1 & 23 & 8 & 0,798 & 0,333 & 0,438 \\
\hline 48 & Woche 16 & Schall & 0 & 0 & 0 & 1 & 23 & 8 & 1,173 & 0,583 & 0,571 \\
\hline 48 & Woche 24 & Schall & 0 & 0 & 0 & 1 & 23 & 8 & 0,881 & 0,25 & 0,643 \\
\hline 48 & Woche 25 & Schall & 0 & 0 & 0 & 1 & 23 & 8 & 0,774 & 0,333 & 0,705 \\
\hline 49 & base & Schall & 0 & 1 & 1 & 1 & 28 & 12 & 1,02 & n.b. & n.b \\
\hline 49 & Tag 0 & Schall & 0 & 1 & 1 & 1 & 28 & 12 & n.b. & 0,227 & 0,529 \\
\hline 49 & Tag 14 & Schall & 0 & 1 & 1 & 1 & 28 & 12 & 0,827 & 0,091 & 0,452 \\
\hline 49 & Woche 8 & Schall & 0 & 1 & 1 & 1 & 28 & 12 & 0,949 & 0,136 & 0,538 \\
\hline 49 & Woche 12 & Schall & 0 & 1 & 1 & 1 & 28 & 12 & 0,929 & 0,136 & 0,587 \\
\hline 49 & Woche 16 & Schall & 0 & 1 & 1 & 1 & 28 & 12 & 0,891 & 0,182 & 0,51 \\
\hline 49 & Woche 24 & Schall & 0 & 1 & 1 & 1 & 28 & 12 & 0,861 & 0,091 & 0,471 \\
\hline 49 & Woche 25 & Schall & 0 & 1 & 1 & 1 & 28 & 12 & 0,861 & 0,091 & 0,471 \\
\hline 50 & base & Hand & 1 & 1 & 0 & 1 & 25 & 0 & 0,82 & n.b. & n.b. \\
\hline 50 & Tag 0 & Hand & 1 & 1 & 0 & 1 & 25 & 0 & n.b. & 0,375 & 0,813 \\
\hline 50 & Tag 14 & Hand & 1 & 1 & 0 & 1 & 25 & 0 & 0,798 & 0,25 & 0,688 \\
\hline 50 & Woche 8 & Hand & 1 & 1 & 0 & 1 & 25 & 0 & 1,762 & 0,292 & 0,714 \\
\hline 50 & Akku leer & Hand & 1 & 1 & 0 & 1 & 25 & 0 & 1,315 & 0,485 & 0,884 \\
\hline 50 & Ende & Hand & 1 & 1 & 0 & 1 & 25 & 0 & 0,756 & 0,333 & 0,759 \\
\hline 50 & Laufz. in $d$ & Hand & 1 & 1 & 0 & 1 & 25 & 0 & 83 & 83 & 83 \\
\hline 51 & base & Hand & 1 & 2 & 0 & 0 & 39 & 15 & 0,13 & n.b. & n.b. \\
\hline 51 & Tag 0 & Hand & 1 & 2 & 0 & 0 & 39 & 15 & n.b. & 0,037 & 0,37 \\
\hline 51 & Tag 14 & Hand & 1 & 2 & 0 & 0 & 39 & 15 & 0,341 & 0 & 0,407 \\
\hline 51 & Woche 8 & Hand & 1 & 2 & 0 & 0 & 39 & 15 & 0,629 & 0,222 & 0,389 \\
\hline 51 & Akku leer & Hand & 1 & 2 & 0 & 0 & 39 & 15 & 0,614 & 0,222 & 0,491 \\
\hline 51 & Ende & Hand & 1 & 2 & 0 & 0 & 39 & 15 & 0,5 & 0,111 & 0,426 \\
\hline 51 & Laufz. in d & Hand & 1 & 2 & 0 & 0 & 39 & 15 & 86 & 86 & 86 \\
\hline 52 & Base & Hand & 1 & 1 & 0 & 1 & 23 & 16 & 0,55 & n.b. & n.b. \\
\hline 52 & Tag 0 & Hand & 1 & 1 & 0 & 1 & 23 & 16 & n.b. & 0,042 & 0,384 \\
\hline 52 & Tag 14 & Hand & 1 & 1 & 0 & 1 & 23 & 16 & 0,679 & 0 & 0,339 \\
\hline 52 & Woche 8 & Hand & 1 & 1 & 0 & 1 & 23 & 16 & 0,637 & 0,083 & 0,446 \\
\hline 52 & Akku leer & Hand & 1 & 1 & 0 & 1 & 23 & 16 & 0,929 & 0,458 & 0,518 \\
\hline 52 & Ende & Hand & 1 & 1 & 0 & 1 & 23 & 16 & 0,708 & 0,25 & 0,295 \\
\hline 52 & Laufz. in d & Hand & 1 & 1 & 0 & 1 & 23 & 16 & 84 & 84 & 84 \\
\hline 53 & Base & Hand & 1 & 0 & 0 & 1 & 24 & 7 & 1,54 & n.b. & n.b. \\
\hline 53 & Tag 0 & Hand & 1 & 0 & 0 & 1 & 24 & 7 & n.b. & 0,65 & 1,313 \\
\hline 53 & Tag 14 & Hand & 1 & 0 & 0 & 1 & 24 & 7 & 1,451 & 0,55 & 1,24 \\
\hline 53 & Woche 8 & Hand & 1 & 0 & 0 & 1 & 24 & 7 & 1,472 & 0,5 & 1,26 \\
\hline 53 & Akku leer & Hand & 1 & 0 & 0 & 1 & 24 & 7 & 1,479 & 0,6 & 1,208 \\
\hline 53 & Ende & Hand & 1 & 0 & 0 & 1 & 24 & 7 & 1,389 & 0,4 & 1,073 \\
\hline 53 & Laufz. in d & Hand & 1 & 0 & 0 & 1 & 24 & 7 & 66 & 66 & 66 \\
\hline 54 & Base & Hand & 1 & 0 & 0 & 0 & 26 & 11 & 1,23 & n.b. & n.b. \\
\hline 54 & Tag 0 & Hand & 1 & 0 & 0 & 0 & 26 & 11 & n.b. & 0,625 & 0,857 \\
\hline
\end{tabular}




\begin{tabular}{|c|c|c|c|c|c|c|c|c|c|c|c|}
\hline 54 & Tag 14 & Hand & 1 & 0 & 0 & 0 & 26 & 11 & 0,946 & 0,417 & 0,848 \\
\hline 54 & Woche 8 & Hand & 1 & 0 & 0 & 0 & 26 & 11 & 1,018 & 0,333 & 0,848 \\
\hline 54 & Woche 12 & Hand & 1 & 0 & 0 & 0 & 26 & 11 & 0,923 & 0,333 & 0,813 \\
\hline 54 & Akku leer & Hand & 1 & 0 & 0 & 0 & 26 & 11 & 1,089 & 0,458 & 1,152 \\
\hline 54 & Ende & Hand & 1 & 0 & 0 & 0 & 26 & 11 & 1,054 & 0,375 & 1,161 \\
\hline 54 & Laufz. in d & Hand & 1 & 0 & 0 & 0 & 26 & 11 & 106 & 106 & 106 \\
\hline 55 & Base & Hand & 1 & 2 & 0 & 0 & 21 & 4 & 0,43 & n.b. & n.b. \\
\hline 55 & $\operatorname{Tag} 0$ & Hand & 1 & 2 & 0 & 0 & 21 & 4 & n.b. & 0,25 & 0,643 \\
\hline 55 & Tag 14 & Hand & 1 & 2 & 0 & 0 & 21 & 4 & 0,423 & 0,208 & 0,607 \\
\hline 55 & Woche 8 & Hand & 1 & 2 & 0 & 0 & 21 & 4 & 0,536 & 0,292 & 0,714 \\
\hline 55 & Akku leer & Hand & 1 & 2 & 0 & 0 & 21 & 4 & 0,554 & 0,292 & 0,83 \\
\hline 55 & Ende & Hand & 1 & 2 & 0 & 0 & 21 & 4 & 0,452 & 0,167 & 0,616 \\
\hline 55 & Laufz. in d & Hand & 1 & 2 & 0 & 0 & 21 & 4 & 85 & 85 & 85 \\
\hline 56 & Base & Hand & 1 & 1 & 1 & 1 & 28 & 4 & 0,46 & n.b. & n.b. \\
\hline 56 & Tag 0 & Hand & 1 & 1 & 1 & 1 & 28 & 4 & n.b. & 0,083 & 0,384 \\
\hline 56 & Tag 14 & Hand & 1 & 1 & 1 & 1 & 28 & 4 & 0,726 & 0,167 & 0,42 \\
\hline 56 & Woche 8 & Hand & 1 & 1 & 1 & 1 & 28 & 4 & 0,702 & 0,125 & 0,438 \\
\hline 56 & Woche 12 & Hand & 1 & 1 & 1 & 1 & 28 & 4 & 0,839 & 0,083 & 0,509 \\
\hline 56 & Akku leer & Hand & 1 & 1 & 1 & 1 & 28 & 4 & 0,827 & 0,125 & 0,554 \\
\hline 56 & Ende & Hand & 1 & 1 & 1 & 1 & 28 & 4 & 0,696 & 0,042 & 0,455 \\
\hline 56 & Laufz. in d & Hand & 1 & 1 & 1 & 1 & 28 & 4 & 89 & 89 & 89 \\
\hline 57 & Base & Hand & 1 & 2 & 1 & 1 & 27 & 10 & 0,55 & n.b. & n.b. \\
\hline 57 & Tag 0 & Hand & 1 & 2 & 1 & 1 & 27 & 10 & n.b. & 0,042 & 0,375 \\
\hline 57 & Tag 14 & Hand & 1 & 2 & 1 & 1 & 27 & 10 & 0,72 & 0,042 & 0,375 \\
\hline 57 & Woche 8 & Hand & 1 & 2 & 1 & 1 & 27 & 10 & 0,643 & 0,083 & 0,473 \\
\hline 57 & Akku leer & Hand & 1 & 2 & 1 & 1 & 27 & 10 & 0,94 & 0,417 & 0,563 \\
\hline 57 & Ende & Hand & 1 & 2 & 1 & 1 & 27 & 10 & 0,696 & 0,25 & 0,384 \\
\hline 57 & Laufz. in d & Hand & 1 & 2 & 1 & 1 & 27 & 10 & 81 & 81 & 81 \\
\hline 58 & Base & Hand & 1 & 1 & 1 & 1 & 30 & 12 & 0,21 & n.b. & n.b. \\
\hline 58 & Tag 0 & Hand & 1 & 1 & 1 & 1 & 30 & 12 & n.b. & 0,042 & 0,286 \\
\hline 58 & Tag 14 & Hand & 1 & 1 & 1 & 1 & 30 & 12 & 0,196 & 0 & 0,339 \\
\hline 58 & Woche 8 & Hand & 1 & 1 & 1 & 1 & 30 & 12 & 0,268 & 0,083 & 0,268 \\
\hline 58 & Akku leer & Hand & 1 & 1 & 1 & 1 & 30 & 12 & 0,292 & 0,042 & 0,259 \\
\hline 58 & Ende & Hand & 1 & 1 & 1 & 1 & 30 & 12 & 0,185 & 0 & 0,313 \\
\hline 58 & Laufz. in $d$ & Hand & 1 & 1 & 1 & 1 & 30 & 12 & 84 & 84 & 84 \\
\hline 59 & Base & Hand & 1 & 0 & 0 & 1 & 20 & 2 & 1,1 & n.b. & n.b. \\
\hline 59 & Tag 0 & Hand & 1 & 0 & 0 & 1 & 20 & 2 & n.b. & 0,042 & 0,321 \\
\hline 59 & Tag 14 & Hand & 1 & 0 & 0 & 1 & 20 & 2 & 0,929 & 0 & 0,268 \\
\hline 59 & Woche 8 & Hand & 1 & 0 & 0 & 1 & 20 & 2 & 1,02 & 0,125 & 0,438 \\
\hline 59 & Akku leer & Hand & 1 & 0 & 0 & 1 & 20 & 2 & 0,982 & 0,125 & 0,51 \\
\hline 59 & Ende & Hand & 1 & 0 & 0 & 1 & 20 & 2 & 0,851 & 0,125 & 0,402 \\
\hline 59 & Laufz. in d & Hand & 1 & 0 & 0 & 1 & 20 & 2 & 84 & 84 & 84 \\
\hline 60 & Base & Hand & 1 & 1 & 0 & 1 & 24 & 8 & 0,3 & n.b. & n.b. \\
\hline 60 & Tag 0 & Hand & 1 & 1 & 0 & 1 & 24 & 8 & n.b. & 0,25 & 0,438 \\
\hline 60 & Tag 14 & Hand & 1 & 1 & 0 & 1 & 24 & 8 & 0,351 & 0,125 & 0,357 \\
\hline 60 & Woche 8 & Hand & 1 & 1 & 0 & 1 & 24 & 8 & 0,53 & 0,167 & 0,455 \\
\hline 60 & Akku leer & Hand & 1 & 1 & 0 & 1 & 24 & 8 & 0,548 & 0,333 & 0,607 \\
\hline 60 & Ende & Hand & 1 & 1 & 0 & 1 & 24 & 8 & 0,494 & 0,208 & 0,51 \\
\hline 60 & Laufz. in d & Hand & 1 & 1 & 0 & 1 & 24 & 8 & 81 & 81 & 81 \\
\hline
\end{tabular}




\begin{tabular}{|c|c|c|c|c|c|c|c|c|c|c|c|}
\hline 61 & Base & Hand & 0 & 1 & 0 & 1 & 23 & 8 & 0,5 & n.b. & n.b. \\
\hline 61 & Tag 0 & Hand & 0 & 1 & 0 & 1 & 23 & 8 & n.b. & 0,65 & 1,49 \\
\hline 61 & Tag 14 & Hand & 0 & 1 & 0 & 1 & 23 & 8 & 1,042 & 0,25 & 1,26 \\
\hline 61 & Woche 8 & Hand & 0 & 1 & 0 & 1 & 23 & 8 & 1,264 & 1 & 1,167 \\
\hline 61 & Woche 12 & Hand & 0 & 1 & 0 & 1 & 23 & 8 & 1,194 & 0,4 & 1,24 \\
\hline 61 & Woche 16 & Hand & 0 & 1 & 0 & 1 & 23 & 8 & 1,424 & 1,3 & 1,448 \\
\hline 61 & Woche 17 & Hand & 0 & 1 & 0 & 1 & 23 & 8 & 1,104 & 0,9 & 1,521 \\
\hline 62 & Base & Hand & 0 & 1 & 0 & 0 & 23 & 6 & 0,68 & n.b. & n.b. \\
\hline 62 & Tag 0 & Hand & 0 & 1 & 0 & 0 & 23 & 6 & n.b. & 0,292 & 0,393 \\
\hline 62 & Tag 14 & Hand & 0 & 1 & 0 & 0 & 23 & 6 & 0,732 & 0,167 & 0,42 \\
\hline 62 & Woche 8 & Hand & 0 & 1 & 0 & 0 & 23 & 6 & 0,827 & 0,083 & 0,295 \\
\hline 62 & Woche 12 & Hand & 0 & 1 & 0 & 0 & 23 & 6 & 0,762 & 0,042 & 0,348 \\
\hline 62 & Woche 16 & Hand & 0 & 1 & 0 & 0 & 23 & 6 & 0,768 & 0,125 & 0,304 \\
\hline 62 & Woche 17 & Hand & 0 & 1 & 0 & 0 & 23 & 6 & 0,792 & 0,083 & 0,33 \\
\hline 63 & Base & Hand & 0 & 1 & 0 & 0 & 23 & 1 & 1,04 & n.b. & n.b. \\
\hline 63 & Tag 0 & Hand & 0 & 1 & 0 & 0 & 23 & 1 & n.b. & 0,083 & 0,652 \\
\hline 63 & Tag 14 & Hand & 0 & 1 & 0 & 0 & 23 & 1 & 0,589 & 0,083 & 0,571 \\
\hline 63 & Woche 8 & Hand & 0 & 1 & 0 & 0 & 23 & 1 & 0,976 & 0 & 0,589 \\
\hline 63 & Woche 12 & Hand & 0 & 1 & 0 & 0 & 23 & 1 & 0,881 & 0,125 & 0,625 \\
\hline 63 & Woche 16 & Hand & 0 & 1 & 0 & 0 & 23 & 1 & 0,857 & 0,167 & 0,491 \\
\hline 63 & Woche 17 & Hand & 0 & 1 & 0 & 0 & 23 & 1 & 0,845 & 0,125 & 0,554 \\
\hline 64 & Base & Hand & 0 & 0 & 0 & 1 & 27 & 11 & 1,13 & n.b. & n.b. \\
\hline 64 & Tag 0 & Hand & 0 & 0 & 0 & 1 & 27 & 11 & n.b. & 0,409 & 0,798 \\
\hline 64 & Tag 14 & Hand & 0 & 0 & 0 & 1 & 27 & 11 & 1,365 & 0,182 & 0,76 \\
\hline 64 & Woche 8 & Hand & 0 & 0 & 0 & 1 & 27 & 11 & 1,205 & 0,273 & 0,808 \\
\hline 64 & Woche 12 & Hand & 0 & 0 & 0 & 1 & 27 & 11 & 1,173 & 0,273 & 0,856 \\
\hline 64 & Woche 16 & Hand & 0 & 0 & 0 & 1 & 27 & 11 & 1,135 & 0,227 & 0,74 \\
\hline 64 & Woche 17 & Hand & 0 & 0 & 0 & 1 & 27 & 11 & 1,058 & 0,182 & 0,731 \\
\hline 65 & Base & Hand & 0 & 2 & 0 & 1 & 58 & 18 & 1,33 & n.b. & n.b \\
\hline 65 & Tag 0 & Hand & 0 & 2 & 0 & 1 & 58 & 18 & n.b. & 0,292 & 0,795 \\
\hline 65 & Tag 14 & Hand & 0 & 2 & 0 & 1 & 58 & 18 & 1,025 & 0,25 & 0,696 \\
\hline 65 & Woche 8 & Hand & 0 & 2 & 0 & 1 & 58 & 18 & 1,117 & 0,292 & 0,768 \\
\hline 65 & Woche 12 & Hand & 0 & 2 & 0 & 1 & 58 & 18 & 0,992 & 0,167 & 0,661 \\
\hline 65 & Woche 16 & Hand & 0 & 2 & 0 & 1 & 58 & 18 & 0,942 & 0,25 & 0,723 \\
\hline 65 & Woche 17 & Hand & 0 & 2 & 0 & 1 & 58 & 18 & 0,908 & 0,125 & 0,67 \\
\hline 66 & Base & Hand & 0 & 0 & 0 & 0 & 60 & 15 & 2,06 & n.b. & n.b. \\
\hline 66 & Tag 0 & Hand & 0 & 0 & 0 & 0 & 60 & 15 & n.b. & 1,318 & 1,067 \\
\hline 66 & Tag 14 & Hand & 0 & 0 & 0 & 0 & 60 & 15 & 1,648 & 0,909 & 0,952 \\
\hline 66 & Woche 8 & Hand & 0 & 0 & 0 & 0 & 60 & 15 & 1,398 & 0,727 & 0,942 \\
\hline 66 & Woche 12 & Hand & 0 & 0 & 0 & 0 & 60 & 15 & 1,324 & 0,545 & 0,854 \\
\hline 66 & Woche 16 & Hand & 0 & 0 & 0 & 0 & 60 & 15 & 1,287 & 0,364 & 0,846 \\
\hline 66 & Woche 17 & Hand & 0 & 0 & 0 & 0 & 60 & 15 & 1,306 & 0,318 & 0,76 \\
\hline 67 & Base & Hand & 0 & 2 & 0 & 1 & 29 & 4 & 0,46 & n.b. & n.b. \\
\hline 67 & Tag 0 & Hand & 0 & 2 & 0 & 1 & 29 & 4 & n.b. & 0,087 & 0,259 \\
\hline 67 & Tag 14 & Hand & 0 & 2 & 0 & 1 & 29 & 4 & 0,383 & 0 & 0,25 \\
\hline 67 & Woche 8 & Hand & 0 & 2 & 0 & 1 & 29 & 4 & 0,352 & 0,043 & 0,306 \\
\hline 67 & Woche 12 & Hand & 0 & 2 & 0 & 1 & 29 & 4 & 0,315 & 0 & 0,231 \\
\hline 67 & Woche 16 & Hand & 0 & 2 & 0 & 1 & 29 & 4 & 0,302 & 0 & 0,296 \\
\hline 67 & Woche 17 & Hand & 0 & 2 & 0 & 1 & 29 & 4 & 0,204 & 0 & 0,222 \\
\hline
\end{tabular}




\begin{tabular}{|c|c|c|c|c|c|c|c|c|c|c|c|}
\hline 68 & Base & Hand & 0 & 2 & 1 & 0 & 54 & 19 & 1,07 & n.b. & n.b. \\
\hline 68 & Tag 0 & Hand & 0 & 2 & 1 & 0 & 54 & 19 & n.b. & 0,571 & 1,02 \\
\hline 68 & Tag 14 & Hand & 0 & 2 & 1 & 0 & 54 & 19 & 0,978 & 0,476 & 0,97 \\
\hline 68 & Woche 8 & Hand & 0 & 2 & 1 & 0 & 54 & 19 & 0,944 & 0,381 & 0,99 \\
\hline 68 & Woche 12 & Hand & 0 & 2 & 1 & 0 & 54 & 19 & 0,989 & 0,333 & 1,04 \\
\hline 68 & Woche 16 & Hand & 0 & 2 & 1 & 0 & 54 & 19 & 0,967 & 0,333 & 0,795 \\
\hline 68 & Woche 17 & Hand & 0 & 2 & 1 & 0 & 54 & 19 & 1,011 & 0,286 & 0,87 \\
\hline 69 & Base & Hand & 0 & 1 & 1 & 0 & 30 & 6 & 0,34 & n.b. & n.b. \\
\hline 69 & $\operatorname{Tag} 0$ & Hand & 0 & 1 & 1 & 0 & 30 & 6 & n.b. & 0,083 & 0,268 \\
\hline 69 & Tag 14 & Hand & 0 & 1 & 1 & 0 & 30 & 6 & 0,274 & 0,083 & 0,205 \\
\hline 69 & Woche 8 & Hand & 0 & 1 & 1 & 0 & 30 & 6 & 0,304 & 0,125 & 0,286 \\
\hline 69 & Woche 12 & Hand & 0 & 1 & 1 & 0 & 30 & 6 & 0,28 & 0,042 & 0,259 \\
\hline 69 & Woche 16 & Hand & 0 & 1 & 1 & 0 & 30 & 6 & 0,256 & 0,042 & 0,33 \\
\hline 69 & Woche 17 & Hand & 0 & 1 & 1 & 0 & 30 & 6 & 0,304 & 0,083 & 0,25 \\
\hline 70 & Base & Hand & 0 & 1 & 1 & 1 & 25 & 10 & 0,66 & n.b. & n.b. \\
\hline 70 & Tag 0 & Hand & 0 & 1 & 1 & 1 & 25 & 10 & n.b. & 0,208 & 0,455 \\
\hline 70 & Tag 14 & Hand & 0 & 1 & 1 & 1 & 25 & 10 & 0,702 & 0,167 & 0,42 \\
\hline 70 & Woche 8 & Hand & 0 & 1 & 1 & 1 & 25 & 10 & 0,744 & 0,25 & 0,482 \\
\hline 70 & Woche 12 & Hand & 0 & 1 & 1 & 1 & 25 & 10 & n.b. & n.b. & n.b. \\
\hline 70 & Woche 16 & Hand & 0 & 1 & 1 & 1 & 25 & 10 & 0,59 & 0,125 & 0,366 \\
\hline 70 & Woche 17 & Hand & 0 & 1 & 1 & 1 & 25 & 10 & 0,607 & 0,167 & 0,429 \\
\hline 71 & Base & Hand & 0 & 2 & 0 & 0 & 22 & 2 & 0,43 & n.b. & n.b. \\
\hline 71 & Tag 0 & Hand & 0 & 2 & 0 & 0 & 22 & 2 & n.b. & 0,125 & 0,42 \\
\hline 71 & Tag 14 & Hand & 0 & 2 & 0 & 0 & 22 & 2 & 0,327 & 0,125 & 0,384 \\
\hline 71 & Woche 8 & Hand & 0 & 2 & 0 & 0 & 22 & 2 & 0,375 & 0,167 & 0,482 \\
\hline 71 & Woche 12 & Hand & 0 & 2 & 0 & 0 & 22 & 2 & 0,464 & 0,25 & 0,795 \\
\hline 71 & Woche 16 & Hand & 0 & 2 & 0 & 0 & 22 & 2 & 0,351 & 0,125 & 0,545 \\
\hline 71 & Woche 17 & Hand & 0 & 2 & 0 & 0 & 22 & 2 & 0,399 & 0,125 & 0,411 \\
\hline
\end{tabular}




\subsection{Tabellenverzeichnis}

Tabelle 1: Gingivaindex und Ausprägungsgrad der gingivalen Entzündung........................ 30

Tabelle 2: $\quad$ Probandencharakteristik für alle Probanden nach Gruppen ................................ 35

Tabelle 3: $\quad$ Gruppenabhängige Mittelwerte (MW) \pm Standardabweichung (SD)

und Medianwerte für den Parameter QHI zu allen

Untersuchungszeitpunkten

Tabelle 4: $\quad$ Paarvergleiche gegen Baseline (Tag 0) für vierwöchigen

Zahnbürstenwechsel

Tabelle 5: $\quad$ Paarvergleiche gegen Baseline (Tag 0) für den sechsmonatigen

Zahnbürstengebrauch

Tabelle 6: $\quad$ Paarvergleiche gegen Baseline (Tag 0) für den sechsmonatigen

Zahnbürstengebrauch

Tabelle 7: $\quad$ Gruppenabhängige Mittelwerte (MW) \pm Standardabweichung (SD)

und Medianwerte für den Parameter PBI zu allen

Untersuchungszeitpunkten

Tabelle 8: $\quad$ Paarvergleiche gegen Baseline (Tag 0) für den vierwöchigen

Zahnbürstengebrauch

Tabelle 9: $\quad$ Paarvergleiche gegen Baseline (Tag 0) für den sechsmonatigen

Zahnbürstengebrauch

Tabelle 10: $\quad$ Paarvergleiche gegen Baseline (Tag 0) für den sechsmonatigen

Zahnbürstengebrauch

Tabelle 11: Gruppenabhängige Mittelwerte (MW) \pm Standardabweichung (SD)

und Medianwerte für den Parameter GI zu allen

Untersuchungszeitpunkten

Tabelle 12: $\quad$ Paarvergleiche gegen Baseline (Tag 0) für den sechsmonatigen

Zahnbürstengebrauch

Tabelle 13: Paarvergleiche gegen Baseline (Tag 0) für den sechsmonatigen

Zahnbürstengebrauch

Tabelle 14: Gruppenabhängige Mittelwerte (MW) \pm Standardabweichung (SD) und Medianwerte für den Parameter QHI zu allen Messzeitpunkten

Tabelle 15: Gruppenabhängige Mittelwerte (MW) \pm Standardabweichung (SD) und Medianwerte für den Parameter PBI zu allen Messzeitpunkten

Tabelle 16: Gruppenabhängige Mittelwerte (MW) \pm Standardabweichung (SD) und Medianwerte für den Parameter GI zu allen Messzeitpunkten . 


\subsection{Abbildungsverzeichnis}

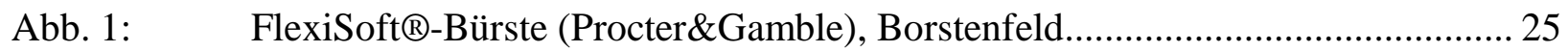

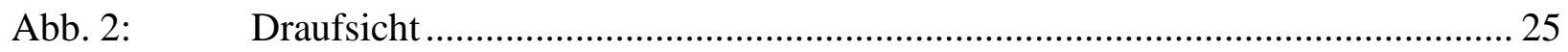

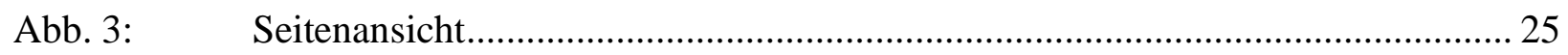

Abb. 4: $\quad$ ProResults-Bürste (Philips), Borstenfeld ....................................................... 26

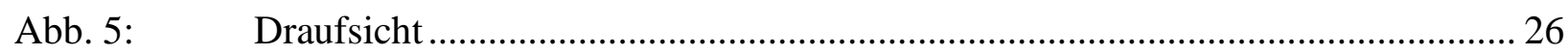

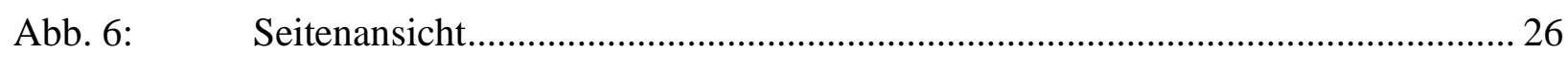

Abb. 7: $\quad$ Dr. Best Vibration ${ }^{\circledR}($ GlaxoSmithKline), Borstenfeld....................................... 27

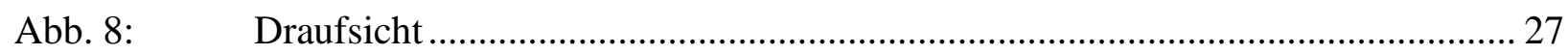

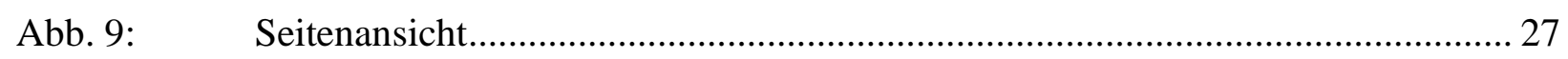

Abb. 10: QHI, Flächeneinteilung des zu bewertenden Zahnes (STEINLE

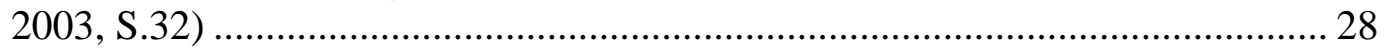

Abb. 11: Graduierung des Plaquebefalls beim QHI (STEINLE 2003, S.32).................... 28

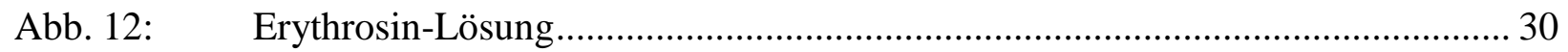

Abb. 13: Graphische Darstellung des Versuchsablaufs im Flussdiagramm ...................... 33

Abb. 14: $\quad$ Verlauf des QHI in den Gruppen A und B. Mittelwerte und 95\%Konfidenzintervalle zu den jeweiligen Messzeitpunkten. Die zu den einzelnen Untersuchungszeitpunkten ermittelten Mittelwerte sind durch eine Linie verbunden.

Abb. 15: $\quad$ Verlauf des QHI in den Gruppen C und D. Mittelwerte und 95\%Konfidenzintervalle zu den jeweiligen Messzeitpunkten. Die zu den einzelnen Untersuchungszeitpunkten ermittelten Mittelwerte sind durch eine Linie verbunden.

Abb. 16: $\quad$ Verlauf des PBI in den Gruppen A und B. Mittelwerte und 95\%Konfidenzintervalle zu den jeweiligen Messzeitpunkten. Die zu den einzelnen Untersuchungszeitpunkten ermittelten Mittelwerte sind durch eine Linie verbunden.

Abb. 17: $\quad$ Verlauf des PBI in den Gruppen C und D. Mittelwerte und 95\%Konfidenzintervalle zu den jeweiligen Messzeitpunkten. Die zu den einzelnen Untersuchungszeitpunkten ermittelten Mittelwerte sind durch eine Linie verbunden.

Abb. 18: $\quad$ Verlauf des GI in den Gruppen A und B. Mittelwerte und 95\%Konfidenzintervalle zu den jeweiligen Messzeitpunkten. Die zu den einzelnen Untersuchungszeitpunkten ermittelten Mittelwerte sind durch eine Linie verbunden.

Abb. 19: $\quad$ Verlauf des GI in den Gruppen C und D. Mittelwerte und 95\%Konfidenzintervalle zu den jeweiligen Messzeitpunkten. Die zu den einzelnen Untersuchungszeitpunkten ermittelten Mittelwerte sind durch eine Linie verbunden. 


\section{Literaturverzeichnis}

ADA (American Dental Association): Acceptance Program Guidelines - Toothbrushes. Council on Scientific Affairs. Chicago, IL 1998

Aguirre A, Levine MJ, Cohen RE, Tabak LA (1987): Immunochemical quantitation of alphaamylase and secretory IgA in parotid saliva from people of various ages. Arch Oral Biol $\underline{32}$, 297-301

Ash MM (1964): A review on the problems and results of studies on manual and power toothbrushes. J Clin Periodontol $\underline{35}$, 202-213

Axelsson P, Lindhe J (1978): Effect of controlled oral hygiene procedures on caries and periodontal disease in adults. J Clin Periodontol $\underline{5}$, 133-151

Bader HI, Boyd RL (1999): Comparative efficacy of a rotary and a sonic powered toothbrush on improving gingival health in treated adult periodontitis patients. Am J Dent 12, 143-147

Barthke A, Brunner E: A nonparametric Alternative to Analysis of Covariance. In: Akritas MG, Politis DN (eds.): Recent Advances and Trends in Nonparametric Statistics. Elsevier, Amsterdam 2003, 109-120

Bass CC (1948): The optimum characteristics of toothbrushes for personal oral hygiene. Dental Items of Interest $\underline{70,697-718}$

Bass CC (1954): An effective method of personal oral hygiene Part I. Louisiana St Med Soc $\underline{106}, 100-112$

Bauch J, Eder-Debye R, Micheelis W: Mundgesundheitszustand und -verhalten in der Bundesrepublik Deutschland. Deutscher Ärzte-Verlag, Köln 1991, 366-376

Bergström J, Lavstedt S (1979): An epidemiologic approach to tooth brushing and dental abrasion. Community Dent Oral Epidemiol 7, 57-64

Bienengräber V, Sponholz H, Hagin J (1995): Abrundungs- und Besteckungsqualität der Borsten fabrikneuer und benutzter Erwachsenen-Zahnbürsten. Dtsch Zahnärztl Z 무, 517-524

Bössmann K (2001): Zahnbürsten und ihr mögliches Infektionsrisiko. Zahnärztl Mitt 22 , 50-52

Bortz J: Statistik für Sozialwissenschaftler. 6.Auflage; Springer Verlag, Berlin 2005

Bowden GH, Hardie JM, Slade GL (1978): Microbiological variations in approximal dental plaque. Caries Res $\underline{15}$ (9), 253

Bratel J, Berggren U (1991): Long-term oral effects of manual or electric toothbrushes used by mentally handicapped adults. Clin Prev Dent $\underline{13}$, 5-7

Bratel J, Berggren U, Hirsch JM (1988): Electric or manual toothbrush? A comparison of the effects on the oral health of mentally handicapped adults. Clin Prev Dent $\underline{10}$, 23-26 
Bunetel L, Tricot-Dolenx S, Agnani G, Bounnaure-Mallet M (2000): In vitro evaluation of the retention of three species of pathogenic micro-organisms by three different types of toothbrush. Oral Microbiol Immunol 15, 313-316

Chava VK (2000): An evaluation of the efficacy of a curved bristle and conventional toothbrush. A comparative clinical study. J Periodontol 71, 785-789

Chilton NW, Fleiss JL (1986): Design and analysis of plaque and gingivitis clinical trials. J Clin Periodontol 13, 400-406

Cochrane Database Syst Rev. 1 (2003): CD002281. Manual versus powered toothbrushing for oral health. Heanue M, Deacon SA, Deery C, Robinson PG, Walmsley AD, Worthington HV, Shaw WC. Cochrane Oral Health Group, MANDEC, University Dental Hospital of Manchester, Higher Cambridge Street, Manchester, UK, M15 6FH

Cochrane Database Syst Rev. 2 (2005): CD002281. Manual versus powered toothbrushing for oral health. Robinson PG, Deacon SA, Deery C, Heanue M, Walmsley AD, Worthington HV, Glenny AM, Shaw WC. Department of Dental Public Health, School of Clinical Dentistry, University of Sheffield, Claremont Crescent, Sheffield, UK

Conforti NJ, Chaves ES, Liebman J, Bowman JP, Warren PR, Cugini M (2001):

A comparative 3-month clinical investigation of the safety and efficacy of a battery-operated and a rechargeable oscillating-rotating power toothbrush. Am J Dent $\underline{14}$, 59-62

Conforti NJ, Cordero RE, Liebman N J, Bowman JP, Putt MS, Kuebler DS, Davidson KR, Cugini M, Warren PR (2003): An investigation into the effect of three months' clinical wear on toothbrush efficacy: results from two independent studies. J Clin Dent $\underline{14}$, 29-33

Cronin MJ, Dembling W, Conforti NJ, Liebman J, Cugini M, Warren PR (2001): A single-use and 3-month clinical investigation of the comparative efficacy of a battery-operated power toothbrush and a manual toothbrush. Am J Dent $\underline{14}, 19-24$

Dahle UR, Tronstad L, Olson I (1993): Spirochaetes in oral infections. Endont Dent Traumatol $\underline{9}, 87-94$

Daly C, Chapple C, Cameron A (1996): Effect of toothbrush wear on plaque control. J Clin Periodontol 23, 45-49

Dean DH (1991): Toothbrushes with graduated wear: correlation with in vitro cleansing performance. Clin Prev Dent $\underline{13}$, 25-30

Dean DH, Beeson LD, Cannon DF, Plunkett CB (1992): Condition of toothbrushes in use: correlation with behavioural and socio-economic factors. Clin Prev Dent $\underline{14}$, 14-18

Dentino AR, Derderian G, Wolf MA, Cugini MA, Johnson R, Van Swol RL, King D, Marks P, Warren P (2002): Six-month comparison of powered versus manual tooth brushing for safety and efficacy in the absence of professional instruction in mechanical plaque control. J Periodontol 73(7), 770-778

Deutsche Industrie Norm (DIN) 13917 / Teil 1/2, Beuth Verlag, Berlin 1979 
Doherty FM, Galustians J, Lim G, MacKay BJ, Sharma NC, Weber DA (1998): Effect of toothbrush wear on plaque removal efficacy. J Dent Res $\underline{77}, 921$

Dunnett CW (1955): A multiple comparison procedure for comparing several treatments with a control. J Am Stat Assoc 50, 1096-1121

Efstratiou M, Papaioannou W, Nakou M, Ktenas E, Vrostos IA, Panis V (2007): Contamination of toothbrush with antibacterial properties by oral micro organisms. J Dent $\underline{35}$, 331-337

Engel D, Nessly M, Morton T, Martin R (1993): Safety testing of a new electronic toothbrush. J Clin Periodontol 64, 941-946

Frandsen A (1985): Changing patterns of attitudes and oral health behaviour. Int Dent J $\underline{35}$, 284-290

Frandsen A, Barbano JP, Suomi JD, Chang JJ, Burke AD (1970): The effectiveness of the Charters `scrub and roll methods of tooth brushing by professionals in removing plaque. Scand J Dent Res $\underline{78}$, 459-463

Frandsen A, Löe H, Kleinman D: Mechanical oral hygiene practices. Dental plaque control measures and oral hygiene practices. IRL Press Limited, Oxford 1986

Glass RT, Lare RR (1986): Toothbrush contamination: a potential health risk? Quintessence Int 17(1), 39-42

Glaze PM, Wade AB (1986): Toothbrush age and wear as it relates to plaque control. J Clin Periodontol 13, 52-56

Golding PS (1982): The development of toothbrush. A short history of tooth cleaning. Dental Health 21, 25-27

Griffith GS, Wilton GMA, Curtis MA, Maiden MFJ, Gillett IR, Wilson DT, Sterne JAC, Johnson NW (1988): Detection of high-risk groups and individuals for periodontal diseases. Clinical Assessment of the Periodontium. J Clin Periodontol 15, 403-410

Grossman E, Proskin H (1997): A comparison of the efficacy and safety of an electric and a manual children's toothbrush. J Am Dent Assoc 128 (4), 469-474

Gülzow HJ: Präventive Zahnheilkunde: Grundlagen und Möglichkeiten der Karies- und Gingivitisprophylaxe.1.Auflage; Hanser Verlag, München 1995

Gunst D: Psychologische Aspekte bei der Auswahl von Handzahnbürsten für Erwachsene. Med. Diss. Mainz 1999

Gustafsson BE, Quensel CE, Lanks LS, Lundqvist C, Grahanen H, Bonow BE, Krasse B (1954): The Vipeholm dental caries study. The effect of different levels of carbohydrate intake oncaries activity in 436 individuals observed for five years (Sweden). Acta Odontol Scand 11, 232 -364

Hannig C, Hannig M (2007): Der initiale orale Biofilm - pathogen oder protektiv? Oralprophylaxe \& Kinderzahnheilkd 29, 73-82 
Hawkins BF, Kohout FJ, Lainson PA, Heckert A (1986): Duration of tooth brushing for effective plaque control. Quintessence Int 17, 361-365

Hedge PP, Ashok KB, Ankola AV (2005): Toothbrush age, wear and plaque control. Indian J Dent $\underline{16}, 61-64$

Heidemann D: Parodontologie.3.Auflage; Urban \& Fischer Verlag, München 1997

Heinrich-Weltzien R, Tawfiq H, Schumann V, Stößer L: Erfurter Kariesrisiko-Studie - Klinische Befunde zur Charakterisierung eines erhöhten Kariesrisikos. In: Stösser L (ed.):

Kariesdynamik und Kariesrisiko. Quintessenz Verlag, Berlin 1998, 179-193

Hellwig E, Klimek J, Attin T: Einführung in die Zahnerhaltung.2.Auflage; Urban \& Fischer Verlag, München 1999

Himmer K, Eickholz P (2008): Elektrische Zahnbürsten - ein Überblick. Quintessenz 59 (11), $1181-1192$

Hingst V (1989): Die Bedeutung der Kontamination von Mundhygieneprodukten. Ergebnisse einer Feldstudie. Zentralbl Bakteriol Mikrobiol Hyg 186, 337-364

Hogan LME, Daly CG, Curtis BH (2007): Comparison of new and 3-month-old brush heads in the removal of plaque using a powered toothbrush. J Clin Periodontol $\underline{34}, 130-136$

Holt J, Sturm D, Master A, Jenkins W, Schmitt P, Hefti AF (2007): A randomized, paralleldesign study to compare the effects of the Sonicare FlexCare and the Oral-B P40 manual toothbrush on plaque and gingivitis. Compend Educ Dent 28, 35-41

Hope CK, Wilson M (2003): Effects of dynamic fluid activity from an electric toothbrush on in vitro oral biofilms. J Clin Periodontol 30, 624-629

Huber B, Rüeger K, Hefti A (1985): Der Einfluss der Zahnreinigungsdauer auf die Plaquereduktion. Schweiz Monatsschr Zahnmed 95, 985-992

Johnson BD, McInnes C (1994): Clinical evaluation of the efficacy and safety of a new sonic toothbrush. J Clin Periodontol $\underline{65}$, 692-697

Jongenelis AP, Wiedemann W (1997): A comparison of plaque removal effectiveness of an electric versus manual toothbrush in children. J Dent Child $\underline{64}$, 176-182

Keyes PH (1960): The infectious and transmissible nature of experimental dental caries. Arch Oral Biol $\underline{1}, 304-320$

Keyes PH (1962): Recent advances in dental caries research. Bacteriological findings and biological implications. Int Dent J $\underline{12}, 443-464$

Khambay BS, Walmsley AD (1995): An in vitro evaluation of electric toothbrushes. Quintessence Int $\underline{26}, 841-848$ 
Klein H, Palmer E, Knutson JW (1938): Studies of dental caries - dental status and dental needs of elementary school children, Public Health Rep Wash $\underline{53}, 751$

Klimm W: Kariologie: Ein Leitfaden für Studierende und Zahnärzte.1.Auflage; Hanser Verlag, München 1997

Kneist S (1998): Zu bislang nicht klassifizierten Begleitphänomenen in der mikrobiologischen Speicheldiagnostik. Oralprophylaxe \& Kinderzahnheilkd 20, 208 - 217

Koch CA, Auschill TM, Arweiler NB (2007): Wann sollte eine Zahnbürste ausgewechselt werden? Oralprophylaxe \& Kinderzahnheilkd 29, 150-158

König KG: Karies und Parodontopathien. Ätiologie und Prophylaxe.1.Auflage; Thieme Verlag, Stuttgart 1987

König KG, Mühlemann HR (1967): The cariogenicity of refined and unrefined sugar in animal experiments. Arch Oral Biol 12, 1297-1298

Kreifeldt JG, Hill PH, Calisti LJ (1980): A systematic study of the plaque removal efficiency of worn toothbrushes. J Dent Res $\underline{59}$, 2047-55

Lang NP: Parodontalerkrankungen - Klassifikation und Charakterisierung.1.Auflage; Quintessenz Verlag, Berlin 2003

Lang NP, Attström R, Löe H: Proceedings of the European Workshop on Mechanical Plaque Control. Quintessence, Chicago 1998

Lange DE: Parodontologie in täglicher Praxis.3.Auflage; Quintessenz Verlag, Berlin 1986

Lange DE, Plagmann HC, Eenboom A, Promesberger A (1977): Klinische Bewertungsverfahren zur Objektivierung der Mundhygiene. Dtsch Zahnärztl Z $\underline{32}$, 44-47

Lazarescu S, Boccaneala A, Illiescu A, De Boever JA (2003): Efficacy of plaque removal and learning effect of a powered and a manual toothbrush. J Clin Periodontol $\underline{30}$, 726-731

Lefkowitz W, Robinson HBG (1962): Effectiveness of automatic and hand brushes in removing dental plaque and debris. J Am Dent Assoc 65, 351-361

Lehmann KM, Hellwig E: Einführung in die restaurative Zahnheilkunde.8.Auflage; Urban \& Schwarzenberg, München 1998

Listgarten MA (1976): Structure of the microbial flora associated with periodontal health and disease in man. J Periodontol $\underline{47}$, 1-18

Löe H, Silness J (1963): Periodontal disease in pregnancy: prevalence and severity. Acta Odontol Scand 21, 532

Löe H, Theilade E, Jensen SB (1965): Experimental gingivitis in man. J Periodontol 36 , $177-187$ 
Malmberg E, Birkhead D, Norvenius G, Noren JG, Dahlen G (1994): Microorganisms on toothbrushes at day-care centers. Acta Odontol Scand $\underline{52}$, 93-98

Mc Cracken GI, Janssen J, Swan M, Steen N, de Jager M, Heasman PA (2003): Effect of brushing force and time on plaque removal using a powered toothbrush. J Clin Periodontol 30, 409-413

Mc Kendrick AHW, McHugh WD, Barbenel L (1971): Toothbrush age and wear. An analysis. Br Dent J 130, 66-68

Miller WD: Die Mikroorganismen der Mundhöhle. Thieme Verlag, Leipzig 1889

Mintel TE, Crawford J (1992): The search for the superior toothbrush design technology. J Clin Periodontol $\underline{3}, 1-4$

Moran JM, Addy M, Newcombe RG (1995): A comparative study of stain removal with two electric toothbrushes and a maual toothbrush. J Clin Periodontol $\underline{6}$, 188-193

Mühlemann HR, Son S (1971): Gingival sulcus bleeding - a leading symptom in initial gingivitis. Helv Odontol Acta $\underline{15}, 107$

Müller HP: Parodontologie.1.Auflage; Thieme Verlag, Stuttgart 2001

Müller HP, Lange DE, Müller RF (1989): Actinobacillus actinomycetemcomitans contamination of toothbrushes from patients harbouring the organism. J Clin Periodontol $\underline{16}$, 388-390

Nielsen, unabhängiges Marktforschungsinstitut, Frankfurt am Main : Schriftliche Mitteilungen 2005

Niemi ML (1987): Gingival abrasion and plaque removal after tooth brushing with an electric and a manual toothbrush. Acta Odontol Scand $\underline{45}$, 367-370

Nies SM, Kröger T, Ansari F, Schaumburg C, Wetzel WE (2008): Keimbesiedlung an Zahnbürsten mit unterschiedlichen Borstenbündelbesteckungen, Oralprophylaxe \& Kinderzahnheilkd 30, 54-60

Noga K, Lange DE, Alai-Omid W (1976): Mykologische Untersuchungen an Zahnbürsten. Dtsch Zahnärztl Z 1ㅗ 396-398

Nolden R: Zahnerhaltungskunde.6.Auflage; Thieme Verlag, Stuttgart 1994

Orland FJ, Blayney JR, Harrison RW, Reyniers JA, Trexler PC, Wagner M, Gordon HA, Luckey TD (1954): Use of germfree animal technic in the study of experimental dental caries. J Dent Res 33, 147-174

Orland FJ, Blayney RJ, Harrison RW, Reyniers JA, Texler PC, Ervin RF, Gordon HA, Wagne $M$ (1955): Experimental caries in germ-free rats inoculated with enterococci. J Am Dent Assoc 50, 259-272

Plagmann HC: Lehrbuch der Parodontologie.1.Auflage; Hanser Verlag, Kiel 1998 
Powers GK, Tussing GJ, Bradley RE (1967): A comparison of effectiveness in interproximal plaque removal of an electric toothbrush and a conventional hand toothbrush. J Clin Periodontol $\underline{5}, 37-41$

Quigley GA, Hein JW (1962): Comparative cleansing efficiency of manual and power brushing. J Am Dent Assoc 65, 26-29

Quirynen M, de Soete N, Pauwels M, Goosens K, Teughels W, van Eldere J, Can Steenberghe D (2001): Bacterial survival rate on tooth and interdental brushes in relation to the use of toothpaste. J Clin Periodontol 28, 1106-1114

Rateitschak KH, Wolf HF, Rateitschak EM: Parodontologie, Farbatlanten der Zahnmedizin1. 3.Auflage; Thieme Verlag, Stuttgart 2004

Rawls HR, Mkwayi-Tulloch NJ, Casella R, Cosgrove R (1989): The measurement of toothbrush wear. J Dent Res $\underline{68}$, 1781-1785

Renggli HR: Ätiologie marginaler Parodontopathien. In: Ketterl W (ed.): Parodontologie. Urban \& Schwarzenberg, München 1990, 33-64

Roulet JF (1995): Präventive Zahnmedizin - Stand der Wissenschaft im Rahmen der Kariologie. Quintessenz 46, 765-781

Sandholm L, Niemi ML, Ainamo J (1982): Identification of soft tissue brushing lesions. A clinical and scanning microscopic study. J Clin Periodontol $\underline{9}$, 397-401

Sauerwein E (1962): Traktat über die Zahnbürste. Dtsch Zahnärztl Z 17, 121-134

Saxer UP, Mühlemann HR (1975): Motivation and education. Schweiz Monatsschr Zahnheilkd $\underline{85}$ (9), 905-919

Saxer UP, Yankell SL (1997a): Impact of improved toothbrushes on dental diseases I. Quintessence Int $\underline{28}, 513-525$

Saxer UP, Yankell SL (1997b): Impact of improved toothbrushes on dental diseases II. Quintessence Int $\underline{28}, 573-593$

Scannapieco FA, Levine MJ: Saliva and dental pellicles. In: Genco RJ, Goldman HM, Cohen DW (eds.): Contemporary Periodontics. Mosby, St. Louis 1990, 117-125

Schaeken M, Sturm D, Master A, Jenkins W, Schmitt P (2007): A randomized, single-use study to compare plaque removal ability of Sonicare FlexCare and Oral-B Triumph Professional Care 9000. Compend Contin Educ Dent 28, 29-34

Schafer F, Nicholson JA, Gerritsen N, Wright RL, Gillam DG, Hall C (2003): The effect of oral care feedback devices on plaque removal and attitudes towards oral care. Int Dent J $\underline{53}$, 404-408

Scheinin A, Mäkinen K (1975): Turku sugar studies V-XIV and XVIII-XX. Acta Odontol Scand 33, 1-400 
Schmid MD, Balmelli OP, Saxer UP (1976): Plaque removing effect of a toothbrush, dental floss and a toothpick. J Clin Periodontol $\underline{3}, 157-165$

Schroeder HE: Pathologie oraler Strukturen: Zähne, Pulpa, Parodont. 3.Auflage; Karger Verlag, Basel 1996

Schweisheimer W (1969): Bevor die Zahnbürste erfunden wurde. Quintessenz 20, 149-150

Serino G, Wennström J, Lindhe J, Eneroth L (1994): The prevalence and distribution of gingival recession in subjects with a high standard of oral hygiene. J Clin Periodontol 21 , 57-63

Seymour GJ (1991): Importance of the host response in the periodontium. J Clin Periodontol $18,421-426$

Sforza NM, Rimondini L, di Menna F, Camorali C (2000): Plaque removal by worn toothbrush, J Clin Periodontol 27, 212-216

Sharma NC, Galustians HJ, McCool JJ (1994): The clinical effects on plaque removal and gingivitis over three-month's use of four complex-design manual toothbrushes. J Clin Dent $\underline{5}$, 114-118

Sharma NC, Galustians HJ, Qaqish H, Cugini MA (1998): A comparison of two electric toothbrushes with respect to plaque removal and subject preference. Am J Dent $\underline{11}$, 29-33

Sicilia A, Arregui I, Gallego M, Cabezas B, Cuesta S (2002): A systematic review of powered vs. manual toothbrushes in periodontal cause-related therapy. J Clin Periodontol 29, 39-54

Splieth C: Professionelle Prävention, Zahnärztliche Prophylaxe für alle Altersgruppen. Quintessenz Verlag, Berlin 2000

Staehle HJ, Kerschbaum T (2003): Mythos Schweiz - Meinungen und Fakten zur Mundgesundheit in der Schweiz im Vergleich zu Deutschland. Dtsch Zahnärztl Z 토, 325-230

Stanford CM, Srikantha R, Wu CD (1997): Efficacy of the Sonicare toothbrush fluid dynamic action on removal of human supragingival plaque. J Clin Dent $\underline{8}, 10-14$

Steinle K: Klinisch-experimentelle Studie über die Auswirkungen zweier Handzahnbürsten auf die Gingiva sowie in Bezug auf die Plaquereduktion an den Zahnoberflächen. Diskussion verschiedener Bewertungsmethoden. Med. Diss. München 2003

Stephan, RM (1944): Intraoral hydrogenion concentrations associated with dental caries activity. J Dent Res 23, 257-266

Taji SS, Rogers AH (1998): ADRF Trebitsch Scholarship. The microbial contamination of toothbrushes. A pilot study. Aust Dent J 느, 128-130

Tan E, Daly C (2002): Comparison of new and 3-month-old toothbrushes in plaque removal. J Clin Periodontol 29, 645-650 
Theilade J: Dental plaque and dental calculus. In: Lindhe J (ed.): Textbook of clinical periodontology. Munksgaard, Kopenhagen 1984, 85

Theilade E, Theilade J, Mikkelsen L (1982): Microbiological studies on early dento-gingival plaque on teeth and mylar strips in humans. J Periodontol Res $\underline{17}$, 12-25

Turesky S, Glickman I, Sandberg R (1972): In vitro chemical inhibition of plaque formation. J Periodontol 43, 263-269

Van der Weijden GA, Timmermann MF, Nijboer A, Lie MA, Van der Velden U (1993): A comparative study of electric toothbrush for the effectiveness of plaque removal in relation to tooth brushing duration. J Clin Periodontol 20, 476-481

Van der Weijden GA, Timmerman MF, Reijerse E (1994): The long term effect of an oscillating/rotating toothbrush on gingivitis. An 8 month clinical study. J Clin Periodontol 21, 86-90

Van der Weijden GA, Timmerman MF, Snoek CM, Reijerse E, Van der Velden U (1996a): Tooth brushing duration and plaque removing efficacy of electric toothbrushes. Am J Dent $\underline{9}$, $31-36$

Van der Weijden GA, Timmerman MF, Reijerse E, Snoek CM, Van der Velden U (1996b): Comparison of an oscillating/rotating electric toothbrush and a 'sonic' toothbrush in plaqueremoving ability. A professional tooth brushing and supervised brushing study. J Clin Periodontol $\underline{23}, 407-411$

Van der Weijden GA, Timmermann MF, Piscaer M, Ijzermann Y, Warren P, Van der Velden U (1998): A comparison of the efficacy of a novel electric toothbrush and a manual toothbrush in the treatment of gingivitis. Am J Dent $\underline{11}, 23-28$

Van Nüss K, Friedl KH, Hiller KA, Hornecker E, Mausberg RF, Ziebolz D (2010): Untersuchung zur Abnutzung von Handzahnbürsten unterschiedlicher Preiskategorien. Schweiz Monatsschr Zahnmed 120, 757-763

Warren DP, Goldschmidt MC, Thomson MB, Adlerstorthz K, Keene HJ (2001): The effects of toothpastes on the residual microbial contamination of toothbrushes. J Am Dent Assoc 132, 1241

Warren PR, Landmann H, Chater BV (1998): Electric toothbrush use: Attitude and experience among dental practioners in Germany. Am J Dent 131, 53-56

Warren PR, Jacobs D, Low MA, Chater BV, King DW (2002): A clinical investigation into the effect of toothbrush wear on efficacy. J Clin Dent $\underline{13}$, 119-124

Wetzel WE, Schaumburg C, Ansari F, Kroeger T, Sziegoleit A (2005): Microbiological contamination of toothbrushes with different principles of filament anchoring. J Am Dent Assoc $\underline{136}, 758-765$

Williams K, Ferrante A, Dockter K, Haun J, Biesbrock AR, Bartizek RD (2004): One and 3minute plaque-removal by a battery-powered versus a manual toothbrush. J Clin Periodontol $\underline{75}, 1107-1113$ 
Wolff L, Dahlèn G, Aeppli D (1994): Bacteria as risk markers for periodontitis. J Periodontol 64, 498-510

Yankell SL, Emling R, Shi X (1997): Interproximal access efficacy of Sonicare Plus and Braun Oral-B Ultra compared to a manual toothbrush. J Clin Dent $\underline{8}$, 26-29

Ziebolz D, van Nüss K, Hornecker E, Mausberg RF (2006): Eine Untersuchung gebrauchter Handzahnbürsten - Ergebnisse einer Umtauschaktion. Oralprophylaxe \& Kinderzahnheilkd $\underline{28}, 54-59$

Zimmer S, Didner B, Roulet JF (1999): Klinische Studie zur Effektivität von elektrischen Zahnbürsten. Prophylaxe-Impuls $\underline{3}$, 164-169 


\section{Danksagung}

An erster Stelle danke ich Herrn Prof. Dr. med. dent. Rainer F. Mausberg, meinem Doktorvater, für die Überlassung des Themas, für sein Mitwirken, seine Hilfsbereitschaft und seine fachlichen Anregungen.

Ich bedanke mich bei Hern Dr. med. dent. Dirk Ziebolz, Mitarbeiter der Abteilung Präventive Zahnmedizin, Parodontologie und Kariologie, der mit seiner vorbildlichen Betreuung und Unterstützung zum Gelingen dieser Arbeit beigetragen hat.

Ebenso möchte mich bei Frau Katharina Lange, Mitarbeiterin der Abteilung Medizinische Statistik, für ihren Beitrag bei der Auswertung der Studienergebnisse bedanken. 


\section{Lebenslauf}

Am 04.03.1981 wurde ich, Sabine Wurbs, in Kassel als zweite Tochter des Zahnarztes Dr. Manfred Wurbs und der Krankengymnastin Heide Wurbs geb. Dach geboren.

Von 1987 bis 1991 besuchte ich die Hupfeldschule in Kassel, von 1991-1998 das Friedrichsgymnasium Kassel. Im Jahr 1998 wechselte ich zur Jacob-Grimm-Schule in Kassel und erlangte dort im Juni 2000 die allgemeine Hochschulreife.

Im Oktober 2001 begann ich das Studium der Zahnheilkunde an der Georg-AugustUniversität Göttingen, legte dort im Dezember 2006 mein Staatsexamen ab und erhielt die Approbation als Zahnärztin.

Anfang des Jahres 2007 begann ich meine Doktorarbeit in der Abteilung Präventive Zahnmedizin, Parodontologie und Kariologie im Zentrum Zahn-, Mund- und Kieferheilkunde der Universitätsmedizin Göttingen unter Betreuung von Prof. Dr. med. dent. Rainer Mausberg und Herrn Dr. med. dent. Dirk Ziebolz.

Von April 2007 bis September 2009 arbeitete ich als wissenschaftliche Mitarbeiterin der Abteilung Präventive Zahnmedizin, Parodontologie und Kariologie der Universitätsmedizin Göttingen, von Oktober 2009 bis Oktober 2010 war ich als wissenschaftliche Mitarbeiterin in der Abteilung Prothetik der Universitätsmedizin Göttingen angestellt. 